\title{
A COMPEND or:
}

Veterinary Materia Mediea AND

\section{Therapeuties}

HASSLOOH 
LIBRARY OF CONGRESS, Chap. S+ Copyright No............. Shelf H 35

UNITED STATES OF AMERICA. 





\section{A COMPEND}

$\mathrm{OF}$

\section{Veterinary Materia Medica}

AND

\section{Therapeuties.}

Dr. A. C. HASSLOCH, V.S.,

Lecturer on Materia Medica and Therapeutics and Professor of Veierinary Dentistry at the New York College of Veterinary Surgeons and School of Comparative Medicine, N. Y.

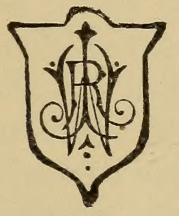

New York :

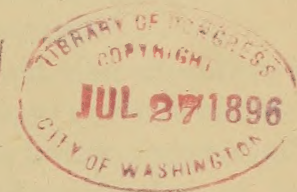

\section{JUL 891896}

WILLIAM R. JENKINS,

VETERINARY PUBLISHER AND BOOKSELLER, 851-853 Siхтн Avenue (48th St.) 
Copyright, 1896, BX WiLliam R. Jenkins.

Ail Rights Reserved.

$$
12-32949
$$

Printed by the

$58^{15} 3^{5}$

Press of William R. Jenkins.

NEW YoRK. 


\section{PREF A CE.}

HAVING recognized the want of a short but comprehensive work embracing the most commonly used veterinary medicines, I have culled the most important facts from the standard works of Bartholow, Brunton, Potter, Finlay Dun, etc., which, together with the experience derived from an extended practice and research, form the basis for this work. It is intended not as an exhaustive treatise of the subject, but as a short and easy reference for the veterinary student, which, in connection with the course of lectures delivered by me at thə New York College of Veterinary Surgeons, will serve to lay a foundation upon which the student in more leisure hours can build a superstructure of more detailed study. For the busy practitioner it will doubtless also serve as a ready reference and aid to the memory.

It has been my effort to pay particular attention to the physiological actions of drugs, to exclude all such as are useless and obsolete, and to follow a systematic and intelligible grouping and classification of the most important remedies.

A very complete index is another feature which will undoubtedly be appreciated by all who use the book.

$$
\text { A. C. Hassloch, V.S. }
$$

New YoRK, February, 1896. 



\section{VETERINARY MATERIA MEDICA AND THERAPEUTICS.}

\section{INTRODUCTION.}

Pharmacology is that science which treats of the drugs employed in medicine, and, therefore, embraces all of materia medica and therapeutics pertaining to drugs. It is supplemented by

PhARMACY, the art of preparing drugs in accordance with the requirements of the pharmacologist and of dispensing them on the prescription of the therapeutist. Pharmacy, therefore, must embrace a thorough knowledge of materia medica, a good idea of the theories and manipulations of chemistry, and an intimate practical experience in many operations peculiar to itself.

MATERIA MEDica is that branch of pharmacology which treats of all substances used as medicines, describing their origin, composition, chemical properties, physical characteristics, modes of preparation and administration, and their physiological and toxicological actions.

It is divided into Pharmacodynamics and ToxiCOLOGY. 
Pharmacodynamics is a treatise on the physiological action of drugs-i.e., the modifying power of drugs upon the normal physiological activity of the animal organism; while

Toxicology tells us what would be the effects of drugs if given in poisonous doses. This also embraces the study of drug antagonists and chemical antidotes.

TheraPeutics is that branch of medical science which considers the application of remedies as a means of cure and alleviation of disease. It includes the discussion of all matters relating to the science and art of healing.

The general term, Therapeutics, includes all the operations of nature, actions of drugs, food, clothing, heat and cold, electricity, etc., and is divided into

\section{Natural Therapeutics and Applied Thera-} PEUTICS.

Natural Therapeutics includes all the processes of nature to heal and alleviate disease independently of art.

Applied Therapeutics embraces the application by art of various agents, foreign to the living organism, to restore the organism to its normal state.

Drugs are material agents of every kind employed in the treatment of disease. This term was formerly applied only to vegetable medicaments in their original form.

$A$ Pharmacopocia is an official list of drugs and their preparations, recognized by the medical profes- 
sion of a certain country. (U. S. P. is revised every ten years.)

A Dispensatory is a commentary upon one or more of the national pharmacopœias, treating in detail of the medicinal substances official * therein as well as of such unofficial $\dagger$ ones as are of especial interest and in general use.

\section{Official Preparations.}

Alkaloids are active, nitrogenous principles existing in plants, from which they are extracted by chemical art. They are organic bases, forming salts with acids, and contain $\mathrm{N}$ with $\mathrm{C}$ and $\mathrm{H}$. Alkaloids are alkaline in reaction, insoluble or but sparingly soluble in water, more readily soluble in alcohol, and their salts are more soluble in water than in any other liquid. In a general way they are very suitable for hypodermic medication.

$\mathrm{AQU}$ \&, waters, aqueous solutions of volatile substances (which may be solids, liquids, or gases). They are dissolved in four ways:

First, by solution in hot or cold water.

Second, by filtration through an absorbent powder.

Third, by percolation through cotton saturated with the substance.

Fourth, by distillation.

Cerata, cerates, are unctuous preparations similar

* Official-according to law.

†Officinal (officina, an office)-so customary and well known as to be found in all shops or offices. 
to ointments, but firmer in consistence. They all contain wax (cera).

Collodia, collodions, are liquid preparations whose basis consists of guncotton dissolved in a mixture of alcohol and ether.

Deсоста, decoctions, are aqueous preparations of vegetable drugs, made by boiling these in water for from fifteen to thirty minutes, and then straining through cloth or muslin. Usually ten per cent. strong, unless otherwise ordered.

ElixIRIA, elixirs, are aromatic sweetened preparations, containing active medicinal agents in small quantity, and are made with a menstruum of alcohol 1 and water 3 . They are intended as an excipient for extracts, salts, etc., and are used mostly in canine practice.

Emplastra, plasters, are solid compounds, tenacious but pliable, and are prepared by incorporating the medicinal agents with certain bases to form a mass, which is to be evenly spread upon chamois skin, muslin, kid, or ordinary adhesive plaster. They are for external use only.

Extracta, extracts, are solid or semi-solid preparations obtained by evaporating solutions of the soluble parts of drugs. Alcohol and water are the most common solvents.

Extracta Fluida, fluid extracts, are alcoholic solutions or concentrated tinctures of vegetable drugs. They are prepared by percolation with menstrua of alcohol, diluted alcohol, or alcohol and water in various proportions; the resulting product being then 
partially evaporated, so that one grain of the drug is represented by one minim of the finished fluid extract.

GlucosideA, glucosides, are organic compounds existing in plants, and belong to the group of neutral principles. They all are resolved into glucose and some other principle when treated with certain reagents or natural ferments. Salicin, santonin, elaterin, etc., are glucosides.

GLYCERITA, glycerites, are mixtures of medicinal substances with glycerin-as glyceritum acidi tannici (twenty per cent.).

INFUSI, infusions, are aqueous preparations of vegetable drugs, using hot or cold water, but without boiling, in the proportion of ten per cent., unless otherwise ordered.

Linimenta, liniments, are thin oleaginous preparations for external use with friction. They are made by dissolving various drugs in oily liquids, or in alcoholic liquids containing fatty oils.

LIQUORES, solutions, include all aqueous solutions of non-volatile substances, except syrups, decoctions, and infusions (liquor gutta-perchæ is an exception; it is a solution of gutta-percha in chloroform).

Misturæe, mixtures, are aqueous preparations of insoluble substances held in suspension by a suitable vehicle.

Mucilagines, mucilages, are thick, viscid liquids, prepared by dissolving gum in water or by extracting with water the mucilaginous principles contained in certain plants. 
Oleata, oleates, are liquid solutions of metallic salts and alkaloids in oleic acid, and are for external use only.

OLEORESIN $A$, oleoresins, are liquid preparations consisting principally of natural oils and resins extracted from vegetable drugs by percolation with stronger ether.

PILULA, pills, are spherical or globular masses composed of medicinal agents, and are intended to be given at one dose. The pill mass consists of the active ingredients and the excipient, which is the substance employed to give the mass its adhesive and plastic qualities.

Pulveres, powders, consist of dry substances in a state of minute subdivision obtained by pulverization. They are sometimes composed of several ingredients which are finely powdered and thoroughly mixed.

RESIN 2 , resins, are solid preparations obtained by precipitating the resinous principles of plants from their alcoholic solutions by the agency of water.

SPIRITUS, spirits, are alcoholic solutions of volatile substances, which may be either solids, liquids, or gases. They may be prepared by simple solution, by solution with maceration, by gaseous solution, by chemical reaction, or by distillation.

SUPPOSITORIA, suppositories, are solid bodies consisting of medicinal substances incorporated with caca' butter, and intended for introduction into the rectum, vagina, or urethra.

SyRUPI, syrups, are concentrated solutions of su- 
gar in water or in aqueous liquids, and are classed as simple, medicated, and flavored.

TINCTUR\&, tinctures, are alcoholic solutions of medicinal substances, all of them being from nonvolatile substances, excepting tincture of iodine. Tinctures are prepared by percolation, maceration, solution, or dilution, the menstrua used being alcohol or diluted alcohol of various strengths, and in some cases the aromatic spirit of ammonia is used.

UnGUENTA, ointments, are soft, fatty mixtures of medicinal agents with a basis of lard, petrolatum, or fixed oils, and are intended for application to the skin by inunction. (Unguentum simplex is lard 80 and yellow wax 20 parts.)

VINA, wines, are a class of tinctures, and differ from these only in so far that the menstruum employed is white wine of a definite alcoholic percentage.

\section{Unofficial Preparations.}

BoLus, bolus or ball, is a solid preparation larger than a pill, to be given at one dose.

BoUgIA, bougies or pencils, are urethral or uterine suppositories, and are made in such shape as to be readily introduced into these canals. The basis is usually a compound of gelatin 3 and glycerin 1 part, melted together.

CAPSUle, capsules, are short tubes of gelatin of such sizes that one will slip over the other, and are used as a means of administering solids or even liquids. 
Cataplasmata, poultices, are applications made to supply heat and moisture to a part. They are sometimes medicated. Farina lini, etc., are usually employed.

Collunarium, is a nasal douche or wash, consisting of various substances dissolved in water with the addition of a small quantity of glycerin.

Collyrium, an eye-wash, an aqueous solution of medicinal substances for application to the eye.

Discus, a disk, is a thin scale of gelatin, medicated for local application to the eye.

EleCtUARIA, electuaries or "pastes," are preparations consisting of various medicinal substances beaten up with honey, syrup, molasses, or glycerin, so as to form a thick paste, and are given with a spoon and smeared upon the tongue and teeth.

EMULSIONES, emulsions, are mixtures containing an oil or a resin minutely subdivided and in a state of suspension in the mixture. This may be accomplished by the aid of some viscid excipient, i.e., gum, soap, alkali, or yolk of egg.

Enemata, enemata or clysters, are liquid preparations for injection into the rectum for various purposes.

Fotus, a fomentation, is a lotion used hot and without friction, and may consist of water alone or may be medicated.

Gargarysma, a gargle, an aqueous solution or mixture for application to the pharyngeal mucous membrane. It usually contains some disinfectant or astringent. 
Haustus, a draught or drench, a liquid mixture intended as a single dose.

INHALATIONES aut VAPORES, inhalations or vapors, are medicines in the form of vapor, gas, or atomized spray, intended for inhalation for their effect upon the respiratory mucous membrane.

INJECTIONES, injections, are aqueous preparations intended for introduction into the cavities of the body by means of a syringe, and are termed according to the part to which they are applied-i.e., urethral, to the urethra, etc.

Lотіо, a lotion or wash, is an aqueous preparation for external use, usually containing some astringent salt. It is applied without friction.

\section{The Administration of Medicines.}

Medicines may be introduced into the circulation by six different methods :

1. Gastro-intestinal Route is mostly used. The remedies after being swallowed are absorbed by the gastro-intestinal blood-vessels and lacteals, and so pass into the circulation.

2. The ReCtum absorbs many substances applied in the form of enemata and suppositories.

3. The Respiratory Tract also rapidly absorbs many medicinal substances on account of its extensive blood supply. These remedies are in the form of vapors or atomized fluids.

4. The Veins and Arteries are sometimes used as a means of introduction for medicines into the sys- 
tem, but usually only in emergencies, as the operation is highly dangerous, especially on the arteries.

5. The Hypodermic Method consists in introducing the medicines into the subcutaneous areolar tissue, from whence they are absorbed by the lymphatics and capillary vessels. This is accomplished by a specially constructed syringe, and when the medicine is delivered deeply into the tissues it is termed a parenchymatous injection.

6. The Skin will absorb many substances, especially if the epidermis or cuticle is removed, and under this route are four subdivisions, viz. :

A. Enepidermic method, by placing the medicine in contact with the epidermis, without friction.

B. Epidermic method consists in the use of friction to hasten the passage of the medicament through the epidermis.

C. Endermic method places the medicament directly upon the derma after removing the cuticle by means of a blister.

$D$. Inoculation method is the introduction of medicinal agents through the scraped or punctured skin.

\section{Classification of Medicines.}

Medicines may be divided in a general way into two great classes, viz., Strmulants and Sedatrves.

A Stimulant is an agent employed to increase the functional activity of a part of the organism. Those stimulants having but a transient effect are called diffusible stimulants. Besides these we designate cerebral, spinal, cardiac, renal, hepatic, and other 
stimulants, according to the special part which they act upon.

A Sedative is an agent which diminishes the functional activity of an organ, lowers motility, and decreases the sensation of pain. In this way a soothing influence is exerted on the system.

Sedatives are divided into two classes, viz., General Sedatives and Local Sedatives.

General Sedatives affect the entire system more or less (this includes narcotics and anæsthetics).

Local Sedatives affect a part only-as pulmonary, spinal, nervous, cardiac sedatives, etc.

Agents Acting on the Nervous System.

Motor excitants, cerebral excitants, deliriants, motor depressants, cerebral depressants, narcotics, hypnotics, analgesics or anodynes, anæsthetics, anti-spasmodics.

Motor Excitants are agents which increase the functional activity of the spinal cord and motor apparatus, producing a heightened reflex excitability and disturbances of motility. Large doses produce tetanic convulsions, and the ultimate result is a motor paralysis from over-stimulation. Nux vomica and ignatia with their alkaloids, strychnine and brucine, ergot, and digitalis belong to this class.

Motor DePREsSants lower the functional activity of the spinal cord and motor apparatus, and when given in larger doses paralyze these directly. Chief members of this group are ether, chloroform, opium, aconite, chloral, lobelia, and belladonna. 
Cerebral-Excitants increase the functional activity of the cerebrum without causing any subsequent depression or suspension of brain function, as camphor, cannabis indica, valerian, cocaine.

Cerebral Depressants suspend or lower the functions of the higher brain after a preliminary stage of excitement. This group includes the narcotics, the general anæsthetics, the hypnotics, and some of the antispasmodics. The action of these is first to stimulate the cerebral functions and then after a time to produce stupor, coma, and insensibility, as alcohol in large doses, opium, bromides, chloral, etc.

DeliRtants excite the functional activity of the brain to such a degree as to disorder the mental faculties. This produces intellectual confusion, loss of will power, delirium, and sometimes convulsions. These agents include belladonna, stramonium, hyoscyamus, chloral, etc.

NARCotics are agents which lessen the relationship of the individual to the outside world (Brunton). They at first excite the higher brain, but soon cause a profound sleep, characterized by increasing stupor; and if the dose has been large enough, coma, insensibility, and finally death by paralysis of the centres of respiration and other functions of organic life. The chief narcotics are opium, cannabis indica, alcohol, belladonna, chloral, chloroform, ether, etc.

HYPNOTICS are agents which produce sleep without causing any preliminary cerebral excitement, as the bromides, paraldehyde, chloralamid, etc. 
ANALGESICS or ANODYNes are agents which relieve pain. This is accomplished in two wayseither by impairing the conductivity of the sensory nerve fibres, or by depression of the cerebral centres of perception and sensation. Opium acts in both ways at the same time, and is therefore the most powerful member of this group.

Anodynes are divided into General Anodynes and LOCAL ANODYNES.

The General Anodynes are taken internally and so act upon the entire organism, as opium, belladonna, henbane, aconite, ether, and chloroform.

Local Anodynes affect the part to which they are applied, generally by direct action upon the nerve endings in the skin and sometimes by reducing the local circulation. These include opium, belladonna, carbolic acid, cocaine, veratrine, aconite, chloroform, etc.

ANAESTHETICS are agents which temporarily destroy sensation.

The General Ancesthetics are volatile substances which when inhaled produce more or less complete unconsciousness and loss of sensation (anæsthesia), and also decreased motor power.

Local Anoesthetics act like the local anodynes but destroy sensation temporarily, while the local anodynes only lessen sensation temporarily-that is, at the part applied.

Chief General Ancesthetics: Ether, methylic ether, chloroform, nitrous oxide, methylene and ethylene bichlorides. 
Chief Local Anoesthetics: Application of extreme cold, ice, ether spray, cocaine, carbolic acid, creosote, hydrocyanic acid, and oil of turpentine.

ANTISPASMODICS are agents which relieve or prevent spasms of voluntary or involuntary muscle in any part of the organism. Their specific modes of action are classed in five divisions :

1. By tonic stimulation of nerve centres, coördination, and circulation-as alcohol, ether, camphor.

2. By direct depression of the motor centres-as amyl nitrite and the bromides.

3. By paralyzing the end organs of the vasomotor nerves-as menthol.

4. By stimulation of the muscular fibres of the intestines to expel gases and flatus-as valerian, asafœtida, and the aromatic oils.

5. By direct depression of the vital functions-as aconite, lobelia, hellebore, and prussic acid.

CoÖRDination oF Movement or maintenance of the equilibrium. The power which controls the actions of the locomotory apparatus is disturbed by a certain class of drugs, especially alcohol. This function is governed by the cerebellum, and in diseases affecting that part of the brain there is a lack of coordination.

\section{Agents Affecting the Organs of Special} Sense.

Mydriatics are agents which cause dilatation of the pupil of the eye. Some act only when applied locally, while others act only when taken internally. 
The principal mydriatic, atropine, will act both locally as well as internally.

Mүотісs are agents which cause contraction of the pupil of the eye-as eserine, pilocarpine, and morphine.

Ocular Sensibility is increased by strychnine, while impairment of vision (amblyopia) due to nerve changes may be temporarily induced by quinine; it may be permanent when caused by lead-poisoning or urea-poisoning.

Excrtability of the Auditory Nerve is increased by strychnine and morphine, which render the sense of hearing more acute; while quinine, salicin, and antipyrine produce hyperæmia of the auditory apparatus, and cause humming or buzzing sounds with diminished sense of hearing.

The Sense of Smell is rendered more acute by strychnine, and depressed by all of the cerebral depressants.

\section{Agents Acting on Respiratory Apparatus.}

Respiratory Strmulants exalt the function of the respiratory centres in the medulla, which make the respirations quicker and deeper-as strychnine, digitalis, ammonia, ether.

Respiratory Depressants lower the action of the respiratory centre, rendering the respirations slow and shallow-as opium in full doses, golsemium, aconite, veratrine, conium, and chloral.

Pulmonary Sedatives relieve cough and dysp- 
nœa by decreasing the irritability of the respiratory centre or by decreasing the irritability of the respiratory nerves-as opium, hydrocyanic acid, cannabis indica, codeine, and amyl nitrite.

EXPECTORANTS are agents which modify the broncho-pulmonary mucous secretion and promote its expulsion. They are divided into NaUseating ExPECTORANTs and Stimulant ExpeCtorants.

Nauseating Expectorants in small doses increase osmosis from the inflamed mucous membrane, while large doses cause vomiting and the mechanical expulsion of the mucus. They also increase secretion generally and lower blood pressure-as ipecacuanha, lobelia, jaborandi, and the antimonial salts.

Stimulant Expectorants.-These are eliminated from the system mainly by the bronchial mucous membranes, which they stimulate in this way, altering the secretion and facilitating expulsion. They increase blood pressure and diminish secretion generally - as the ammonium salts, squills, senega, the turpentines, and balsams of Peru and tolu.

Ciliary Excitants promote expulsion of the bronchial mucus by reflex excitation of the tracheal and bronchial cilia when dissolved in the mouth. This group includes potassium chlorate and the chlorides of ammonium and sodium.

\section{Agents Acting on the Circulation.}

Cardiac Stimulants are such agents as rapidly increase the force and frequency of the pulse in de- 
pressed conditions of the cardiac apparatus. The most rapid and energetic of this class are ether, alcohol, ammonia, and the application of heat.

Cardiac Tonics stimulate the cardiac muscle, slowing and lengthening the contractions if given in moderate doses; but in large doses produce irregularity of the heart's action and may cause death by syncope if toxic doses have been given. The most powerful of this class is digitalis, then convallaria, and strophanthus. Also caffeine and strychnine.

Cardiac Sedatives decrease both force and frequency of the heart's action, and are used to control palpitation and over-action of that organ. The chief members of this class are aconite, veratrum viride, and digitalis.

Vascular Stimulants dilate the peripheral vessels and increase the peripheral circulation; this equalizes blood pressure and so prevents and relieves internal congestions-as alcohol, ether, nitrites, nitrous ether, liquor ammonii acetatis, and heat applied locally by poultices.

Vascular Tonics and Sedatives increase the contractile power of the arterioles, which decreases the capillary circulation and raises blood pressure-as ergot, digitalis, opium in small doses, and cold applied locally.

Agents Acting on the Digestive Apparatus.

Sialogogues are agents which promote the secretion and flow of saliva-as ether, chloroform, mus- 
tard, ginger, pilocarpus, ipecac, mercurials, and antimonials.

Antisialics diminish the secretions of the salivary and buccal glands-as atropine, opium, borax, alkaline salts, and lithia.

REFRIGERANTS impart a sensation of coldness and thereby allay thirst. These include the mineral and vegetable acids, ice, and cold water.

Gastric Tonics or Stomachics increase the appetite and promote gastric digestion. Some act by stimulating the production of the gastric juice, as the alkalies before meals; others stimulate the local circulation, as the aromatic oils, bitters, and alcohol; and some by stimulation of the nerve supply of the stomach, as nux vomica and arsenic.

ACIDS are agents which in concentrated form act as caustics and destroy the tissues; but when properly diluted and given internally in medicinal doses check the production of glands having an acid secretion if coming in contact with the mouths of their ducts, and increase the production of glands having an alkaline secretion. Therefore a dilute acid given before a meal will check the production of the acid gastric juice, but will stimulate the alkaline pancreatic juice. Principal acids-Mineral: Nitric, phosphoric, sulphuric, and hydrochloric. Vegetable: Acetic, citric, and benzoic.

AlKalies, or ANTACIDS, are agents which neutralize acids, act as escharotics upon the tissues, check alkaline and stimulate acid secretions when in contact with the mouths of the ducts of glands producing 
these. Thus a dilute alkali given before a meal will stimulate the production of the acid gastric juice, and check the secretion of the alkaline pancreatic juice. Antacids are subdivided into two classes:

Direct Antacids. - These lessen acidity within the stomach-as liquor potassæ, lime water, liquor sodæ, chalk, carbonates and bicarbonates of $\mathrm{K}, \mathrm{Na}$, $\mathrm{Li}, \mathrm{Mg}$, and $\mathrm{NH}_{4}$, and aromatic spirit of ammonia.

INDIRECT OR REMote ANTACIDS.-These become oxidized in the blood, are excreted in the urine as carbonates, and in this way lessen the acidity of the urine; as liquor sodæ, lithium citrate, liquor potassæ, potassium acetate, citrate, tartrate, and bitartrate, sodium acetate and citrate, carbonates and bicarbonates of $\mathrm{K}, \mathrm{Na}, \mathrm{Li}, \mathrm{Mg}$, and $\mathrm{NH}_{4}$.

Emetics are agents which cause vomiting. They are classed as :

Local Emetics, which act by irritating the end organs of the gastric, œsophageal, or pharyngeal nerves, and by reflex irritation of the vomiting centre-as alum, mustard, salt, and the sulphates of zinc, copper, and mercury.

General or Systemic Emetics, which act by direct irritation of the vomiting centre in the medulla through the medium of the circulation-as ipecac, apomorphine, and tartar emetic.

Anti-Enetics are agents which prevent and relieve vomiting. They are classed as:

Local Gastric Sedatives, which act upon the end organs of the gastric nerves-as arsenic, bismuth, cocaine, ice, etc. 
General Sedatives, which act by reducing irritability of the vomiting centre in the medulla-as amyl nitrite, chloral, bromides, hydrocyanic acid, and nitroglycerin.

CaRminatives favor the expulsion of gases from the stomach and intestines by stimulating peristalsis and the circulation-as asafœtida, capsicum, fennel, camphor, ginger, mustard, pepper, and most of the aromatic oils.

Cathartics or Purgatives are agents which increase or hasten the intestinal evacuations. They are divided into sereral groups, according to degrees and modes of action: Laxatives or aperients, simple purgatives, drastic purgatives, saline purgatives, hydragogue purgatives, cholagogue purgatives.

1. LAXATIVES or APERIENTS, whose action is only moderate-as sulphur, magnesia.

2. Simple Purgatives, which cause active peristalsis and some irritation and griping. These include aloes, linseed oil, castor oil, rhubarb, calomel, and senna.

3. Drastic Purgatives act still more powerfully than the simple purgatives. They cause large watery evacuations, with considerable griping and tenesmus. The chief members of this group are croton oil, gamboge, scammony, elaterium, colocynth, and jalap.

4. Saline Purgatives increase peristalsis, promote osmosis, stimulate the glands, and thus produce free watery evacuations. These include Epsom salt, Glauber salt, chloride of sodium, etc.

5. Hydragogue Purgatives are the most active 
of all the cathartics. They remove from the system a large quantity of water-as croton oil and gamboge.

6. Cholagogue Purgatives stimulate the flow of bile. These include aloes, podophyllin, rhubarb, and mercurials.

INTESTINAL ASTRINGENTS contract the intestinal vessels, diminishing the exudation therefrom, and lessening the fluidity of the fecal discharges. These include the diluted mineral acids, lead acetate, alum, tannic and gallic acids, sulphate of copper, and the per-salts of iron, which also act as astringents to the intestinal mucous membranes.

Cholagogues and Hepatic Stimulants.These are two groups of agents which have a marded selective action upon the biliary secretion.

The Hepatic Stimulants increase the functional activity of the liver cells and also increaset he amount of bile secreted. Nitric acid, nitromuriatic acid, ammonium chloride, bicarbonate of soda, ipecac, and antimony.

Cholagogues remove the bile from the duodenum and prevent its reabsorption by the portal vessels. Aloes, arsenic, rhubarb, sodium phosphate, and sulphate.

Hepatic functions other than the biliary are stimulated by the following-named drugs, viz. :

The Glycogenic Function is stimulated and the production of glycogen increased by sodium bicarbonate, amyl nitrite, and nitromuriatic acid.

Urea is increased by arsenic, antimony, ammonium chloride, iron, and phosphorus. 
Hepatic Depressants decrease the functional activity of the liver. Its various functions are acted upon by different drugs: Bile production decreased by opium, quinine, alcohol, and lead. Grlycogen production decreased by opium, phosphorus, and arsenic. Urea decreased by opium, morphine, colchicum, alcohol, and quinine.

ANTHELMINTICS are agents which destroy (vermicides) or expel (vermifuges) worms inhabiting the intestinal canal. These are classed according to the particular worm they are most efficient against; thus:

Thread-worm : Enemata of alum, sulphate of iron, lime water.

Round-worm : Santonin, areca nut, chenopodium, sulphate of copper, etc., internally, combined with a purgative.

Strongylus micrurus (of sheep) : Injections of turpentine intratracheally.

Tape-worm: Filix mas, kamala, kousso, granatum, turpentine, and chloroform.

\section{Agents Affecting Metabolism.}

Restoratives are agents which promote constructive metamorphosis. They may be subdivided into foods, hæmatics, and tonics; many stimulants are also classed as restoratives.

Foods supply material to maintain the vital processes or to renew some structure of the body.

Hoematics enrich the quality of the blood by in- 
creasing the quantity of its hæmatin. The best known hæmatics are compounds of iron and manganese.

Tonics improve the tone of the tissues on which they have specific action, increasing the vigor of the entire system-as strychnine, quinine, iron, and the vegetable bitters.

Alteratives are agents which alter the course of morbid conditions, modifying the nutritive processes while promoting waste, and in this manner indirectly helping to relieve from disease. Mercury, arsenic, iodine, and their preparations are typical alteratives.

Resolvents or Discutients promote the absorption of morbid products by stimulating the lymphatic system. These include mercury, cadmium, iodine, arsenic, and such local measures as poulticing and counter-irritation.

ANTIPYRETICS reduce high bodily temperature. This may be accomplished by five different methods, viz. :

1st. By diminishing tissue change-quinine, antipyrine, salicin.

2d. By reducing the circulation-aconite, digitalis, antimony. (N. B.: These two lessen heat production.)

3d. By dilating cutaneous vessels, which increases heat radiation-alcohol, sweet spirits of nitre.

4th. By promoting perspiration, its evaporation lowering the temperature-antipyrine, nitrous ether, antimony.

5th. By abstracting heat from the body-cold 
baths, wet pack, cold drinks. (N.B.: These last three promote heat loss.)

AnTIPHLOGISTICs include all measures adopted to reduce inflammation, whether medicine internally or local applications-mercury, opium, aconite, digitalis, ergot, venesection, purgation, counter-irritation, cold, ice, rest, etc.

ANTIPERIODICS, agents which check or prevent the return of various periodically recurring diseasesquinine, arsenic, salicin, eucalyptol.

\section{Agents ACting on Excretion.}

DiURETICS increase the activity of the kidneys, stimulating them to excrete the urine more rapidlyspiritus ætheris nitrosi, digitalis, etc.

Renal Depressants decrease the activity of the renal cells, and diminish or suspend the excretion of urine-morphine, quinine, ergot.

URINARY ALKALIZERS, when taken internally, cause the urine to have an alkaline reaction-alkalies, salts of potassium and lithium.

URINARY ACIDIFIERS render the urine of an acid reaction-benzoic and salicylic acids.

Vesical Tonics stimulate the contractile power of the bladder by increasing the tone of the muscular fibre composing the walls of that organ-strychnine, cantharis, belladonna.

Vesical Sedatives relieve irritability of the bladder and decrease the desire to micturate-opium, cannabis, belladonna. 
Urinary Sedatives relieve irritability along the entire urinary tract through the medium of the urine, which holds them in solution, coming in contact with the genito-urinary mucous membrane-potassium and lithium salts, cubebs, copaiba, sandalwood oil.

ANTILITHICS AND LITHONTRIPTICS are agents which prevent the formation of concretions in the ducts (antilithics), or dissolve these when already formed (lithontriptics) - salts of potash and lithia for uric-acid calculi; lactic acid, dilute nitromuriatic acid, for oxalate of calcium calculi; benzoic acid, dilute nitric acid, for phosphatic calculi.

A Diluent is an indifferent substance, which when absorbed passes through the body, diluting its fluids and excretions-as water.

DIAPHORETICS and SUDORIFICS increase the action of the skin and promote the sweating. They are divided into three groups:

1. Simple diaphoretics enter the circulation and stimulate the sudoriferous glands during their elimination-as jaborandi, nitrous ether, alcohol.

2. Nauseating diaphoretics produce relaxation and dilatation of the capillaries; as tartar emetic, Dover's powder, ipecac, hot baths.

3. Refrigerant diaphoretics reduce the force of the circulation-as ammonium and potassium salts, aconite, ether, nitrites.

ANHIDROTICS check perspiration-atropine, ergot, acids; and locally, cold, opium, etc. 
Agents Acting on the Generative Apparatus.

ApHrodisiacs stimulate the sexual desire and function by direct and reflex action upon the genital centres in the brain and spinal cord-nux vomica, cannabis, cantharis, phosphorus.

ANAPHRODISIACS diminish the sexual appetite and lower its functional power by depressing the genital centres or by decreasing the local circulation-cocaine, belladonna, lupulin, bromides.

Emmenagogues include all measures and remedies which restore the menstrual function. They are divided into:

Direct Emmenagogues, which stimulate the uterine muscular fibres and are ecbolic in large dosesergot, rue, savine, borax, quinine, potassium permanganate.

Indirect Emmenagogues, which act by improving the blood and toning up the nervous system-iron, manganese, strychnine, cod-liver oil.

ECBOLICS or OxYTocics are agents which produce abortion by stimulating the gravid uterus to contract, or by direct irritation of the uterine centre in the cord-ergot, savine, borax, quinine, pilocarpine, cotton-root bark.

Any violent purgative or gastro-intestinal irritant may produce abortion by reflex action.

Uterine Depressants lower the activity of the nervo-muscular apparatus by controlling uterine con- 
tractions-opium, cannabis, bromides, chloral, chloroform.

Uterine Tonics and Alteratives are remedies which act upon the nutrition and functions of the uterus.

Uterine Tonics: Potassium bromide, viburnum opulus, potassium chlorate, viburnum prunifolium, helonias, cimicifuga, pulsatilla, iodine. Also astringents locally.

Uterine Alteratives: Iodine, iodoform, iodol, iodized phenol or carbolated iodine, glycerin, hydrastis, silver nitrate, galvanism.

Galactagogues increase the lacteal secretionjaborandi.

Galactophyga decrease or check the lacteal secretion-belladonna or atropine locally and internally.

\section{Agents Acting on the Skin.}

IRRITANTS are agents which, on application to the skin, cause more or less vascular excitement, and are called counter-irritants when used for exciting a reflex action upon some part of the organism remote from the place of application. They are divided into three groups, according to the severity of their action-viz., Rubefacients, epispastics or blisters, pustulants.

Rubefacients cause only a redness of the skin. Mustard, capsicum, camphor, iodine, turpentine.

Epispastics, Vesicants, OR Blisters produce marked inflammation of the skin and an outpouring 
of serum between the derma and epidermis, thus constituting the blisters-cantharides, euphorbium, mezereon.

Pustulants cause pustules and usually affect only isolated parts of the skin-croton oil, tartar emetic, silver nitrate.

CaUstics or Escharotics are agents which destroy the tissues to which they are applied, by abstracting the water from these tissues or by corrosive oxidation-caustic potash, lime, zinc chloride, silver nitrate, mineral acids.

ASTRINGENTS cause contraction of muscular fibre and lessen secretion from mucous surfaces.

Systemic Astringents act on the internal organs through the circulation-viz., gallic acid.

Local Astringents act upon the part to which they are applied-tannin, alum, salts of copper, lead, zinc, per-salts of iron.

STYPTICS are local applications intended for checking bleeding - as astringents of all kinds, cold, ice, etc.

Hemostatics are agents given internally to stop hemorrhage-dilute mineral acids, gallic acid, ergot, digitalis, lead acetate, and oil of turpentine.

Emollients and Demulcents are substances calculated to soften, soothe, and protect tissues to which they are applied.

Emollients are applied to act externally-as oils, fats, vaseline, starch, glycerin, flaxseed, or other poultices.

Demulcents are used for the same purpose internally. 
Protectives are substances applied to cover or protect an injured or inflamed part to exclude air, dust, water and to prevent friction.

\section{Agents Acting on Microbes, Parasites, etc.}

Antizymotics, agents which prevent and check all organic fermentative processes, also those dependent upon organized ferments (bacteria, cocci, etc.). They are divided into two groups, viz. :

Antiseptics, which destroy the bacilli which cause septic decomposition-as mercuric chloride, hydrogen dioxide, formalin, mercurous chloride, hydronaphthol, sulphurous acid, quinine, thymol, carbolic acid, alcohol, and many others.

Disinfectants are agents which destroy the germs of infectious diseases. Some of these are oxidizan' - lime, chlorine, iodine; some are desulphurantsas sulphate of iron; absorbents-as charcoal; deodorants-as ozone, lime, chlorine, and sulphurous acid gases, which destroy the bad odors while also disinfecting.

PARASITICIDES are agents which destroy animal and vegetable parasites living upon the body. They are applied usually as lotions and ointments and contain sulphur, iodine, mercury and its chlorides, carbolic acid, preparations of tar, etc.

\section{Agents Acting upon Each Other.}

Antidotes are agents which act upon poisons in such \& manner as tr prerents them from exerting their 
toxic properties. This takes place in the alimentary canal, and includes such measures as emetics, stomach-pump, purgatives, etc.

ANTAGONISTS are agents whose action counteracts the effects produced by poisons upon the organism. This action takes place within the blood and tissues, and is applicable mostly to vegetable poisons. This may include artificial respiration, electric treatment, cold douches, motion, rest, etc. 


\section{TABLE OF DOSES}

HoRse.

CatTle

3 years up $=1=2$ years up.

$1 \frac{1}{2}$ years to 3 years $=\frac{1}{2}=1$ year to 2 years

9 months to 18 months $=\frac{1}{4}=\frac{1}{2}$ year to 1 year.

$4 \frac{1}{2}$ months to 9 months $=\frac{1}{8}=3$ months to 6 months

1 month to $4 \frac{1}{2}$ months $=\frac{1}{16}=1$ month to 3 months.

SHEEP.

DoG.

2 years up $=1=\frac{1}{2}$ year to 1 year up.

1 year to 2 years $=\frac{1}{2}=3$ months to 6 months.

$\frac{1}{2}$ year to 1 year $=\frac{1}{4}=1 \frac{1}{4}$ months to 3 months.

3 months to 6 months $=\frac{1}{8}=20$ days to 45 days.

1 month to 3 months $=\frac{1}{16}=10$ days to 20 days.

Pig and Goat.

$1 \frac{1}{2}$ years up $=1$

9 months to 18 months $=\frac{1}{2}$ $4 \frac{1}{2}$ months to 9 months $=\frac{1}{4}$

$2 \frac{1}{2}$ months to $4 \frac{1}{2}$ months $=\frac{1}{9}$

1 month to 2 months $=\frac{1}{18}$
If cattle and horses take 16 , The pig takes 3 .

The goat takes 3 , The dog takes 1 , The cat takes $\frac{1}{2}$.

The dose for the adult dog $=$ the dose for adult man.

Fluids, for cattle usually the samc dose as for the horse.

Solids, for cattle usually $1 \frac{1}{2}$ times the dose for the horse. 


\section{RESTORATIVE AGENTS.}

All agents producing constructive metamorphosis of tissue are included in this division-as the digestive ferments which aid digestion, foods, tonics, hæmatics; also a number of the so-called stimulants.

\section{Digestive Ferments.}

Pepsin and pancreatin, both organic substances, are the most important of these; papain, papaw or papayotin, a ferment obtained from the milky juice of papaya carica, a South American fruit-tree.

Pepsin is an organic ferment which constitutes the digestive principle of the gastric juice of animals. It is usually obtained from the mucous membrane of the pig's stomach.

Pancreatin is obtained from the pancreas of animals and contains four ferments-viz., trypsin, pancreatic diastase, an emulsifying and a milk-curdling ferment.

\section{Preparations.}

Pepsinum Purum, pure pepsin, in yellowish translucent scales soluble in water. Dose: H., gr. xv.3 i.; D., gr. i.-iij.

Pepsinum Saccharatum (U. S. P.), saccharated pepsin, is a mixture of pure pepsin with sugar of milk. Dose: H., 3 ij.-vi.; D., gr. v. 3 ss., after feeding. 
Liquor Pepsini (U. S. P.), solution of pepsin, liquid pepsin. Dose: D., fl. 3 i.-ij. (Contains saccharated pepsin 40 , hydrochloric acid 12 , glycerin 400 , water enough to make 1,000 .)

Extractum Pancreaticus, or pancreatin, which should contain the four pancreatic ferments. Doses: H., 3 ss.-iss. ; D., gr. iij.-v.

Lactopeptin, a powder containing pepsin, pancreatin, diastase, lactic acid, and hydrochloric acid. Dose: D., gr. v. -xv.

INCOMPATIBLES.-Alkalies and many mineral salts precipitate pepsin, while alcohol destroys its activity. Alkalies promote the action of pancreatin.

Physiological ACtion.-Pepsin digests the proteids or nitrogenous principles of the food (albumin, casein, fibrin, etc.), converting these into peptones for assimilation. Pancreatin, in addition to this, also emulsifies the fats and oils, and may be itself digested by pepsin; hence it but seldom reaches the duodenum in its original form. Papain is a powerful digester of fibrin and will act in solutions of any reaction, while pepsin requires an acid solution.

TheraPeUtics.-The internal use of pepsin and pancreatin is limited to young herbivora while receiving milk, and to dogs or other carnivora.

Pepsin is beneficial in atonic dyspepsia, gastralgia, anæmia, chlorosis, and the diarrhœa of calves, foals, and dogs. It may be added to nutritive enemata to assist in their assimilation, the rectum not being a digestive organ. Acidulated solutions may be injected into the substance of morbid growths, 
especially fatty tumors; also to dissolve out the secretive membrane of quittor pipes and similar fistulæ.

Pancreatin and its preparations are used to partly digest milk, gruel, soups, and other foods before administration in cases where there is great digestive debility. All nutritive enemata should be properly peptonized before use.

Papain is a rapid solvent of false membranes and intestinal worms, but is very seldom given internally, as it might digest the gastric mucous membrane itself. It has been successfully used for the destruction of various tumors, but causes considerable pain and febrile reaction.

\section{Mineral Acids.}

Acidum Sulphuricum Dilutum, strength $\frac{1}{10}$. H., fl. 3 i.-ij.; D., Tl x.-xx.

Acidum Sulphuricum Aromaticum, elixir of vitriol, aromatic sulphuric acid, strength $\frac{1}{5}$. Contains sulphuric acid, alcohol, ginger, and cinnamon. This preparation is really an ether resulting from the reaction of the sulphuric acid upon the alcohol. $H$., fl. 3 ss.-i.; D., Tl v.-x.

Acidum Hydrochloricum Dilutum, contains ten per cent. absolute acid. H., fl. 3 i.-ij.; D., m x.-xx. Acidum Nitricum Dilutum, strength $\frac{1}{6}$. H., fl. 亏 i.-ij.; D., m x. $-\mathrm{xx}$.

Acidum Nitro-Hydrochloricum (Dilutum), contains nitric acid 4, hydrochloric acid 15, water 76 . H., fl. 3 i. $-\mathrm{ij}$.; D., Tl x. $-\mathrm{xx}$. 
Acidum Phosphoricum Dilutum, strength $\frac{1}{5}$. H., fl. 3 i.-ij.; D., Tl x.-xx.

These dilute acids should be given well diluted.

Sulphuric Acid, acidum sulphuricum, oil of vitriol $-\mathrm{H}_{2} \mathrm{SO}_{4}-$ is produced by the burning of sulphur and the oxidation and hydration of the resulting sulphurous acid gas by means of nitrous and aqueous vapors. It is a thick, oily-looking, colorless, odorless liquid, of an intensely acid taste and strong acid reaction. Specific gravity, 1.843. It absorbs moisture from the air, and when mixed with water it evolves heat.

Muriatic Acid, acidum hydrochloricum, spirit of salt- $\mathrm{HCl}$. A clear, colorless, intensely sour liquid, having a strong acid reaction, and specific gravity 1.16. It emits white, pungent fumes of the gas which forms about thirty-two per cent. by weight of the solution. Obtained mostly as a by-product in the manufacture of sodium carbonate from common salt.

Nitric Acid, acidum nitricum, aqua fortis, $\mathrm{HNO}_{3}$. A clear, colorless, corrosive, intensely sour liquid, which emits pungent, suffocating, nitrous fumes, and oxidizes, corrodes, and dissolves most organic substances. It has great affinity for water and evolves heat when mixed with this. Nitric acid is prepared from sodium or potassium nitrate, sulphuric acid, and water. It contains about seventy per cent. of true nitric acid, and has the specific gravity 1.42 .

Nitro-Hydrochloric Acid, acidum nitromuriaticum, 
aqua regia. This is a mixture of the nitric and hydrochloric acids, but in its full strength is used only in the arts.

Phosphoric Acid, acidum phosphoricum, $\mathrm{H}_{3} \mathrm{PO}_{4}$. Concentrated phosphoric acid is obtained by heating phosphorus with dilute nitric acid until nitrous fumes no longer come off; the resulting compound is then diluted with water till its specific gravity is 1.5 . It requires about thirty-three per cent. of water. Concentrated phosphoric acid is a clear, colorless, acid, syrupy liquid, and in its purity is not used medicinally.

\section{Physiological Actions of the Mineral Acids.}

The strong acids are corrosive irritant poiscns if given internally, unless properly diluted and in proper quantity. Medicinal doses are refrigerant, antiseptic, tonic, and astringent. Externally: Stimulant, astringent, and escharotic. They abstract water from the tissues, combine with bases to form salts, destroy protoplasm, and are very diffusible. Sulphuric acid carbonizes (black); nitric acid tans (yellow). When in contact with the mouths of ducts having an alkaline secretion this is produced in increased quantity, while if in contact with the mouths of ducts having an acid secretion this latter is decreased. Secretion in general is promoted by nitric acid, and decreased by sulphuric acid-nitric acid aiding peristalsis, sulphuric acid producing constipation. Muriatic acid aids digestion and stimulates mucous and intestinal secretions, especially when com- 
bined with bitters. All of the mineral acids tend to check fermentation and aid in digestion. If continued for too long a time they lessen the production of gastric juice, and thus impair digestion. Phosphoric acid is the most agreeable of the mineral acids and is not so apt to derange digestion if given for a longer period. The mineral acids are excreted mainly by the kidneys, diminishing the alkalinity or increasing the normal acidity of the urine.

Toxic doses of the mineral acids produce violent gastro-enteritis; lips, mouth, and fauces are highly inflamed and swollen; respiration more labored as the swelling of the throat increases; pulse weak and quick; great abdominal pain, increasing prostration, and death in from three to twelve hours.

Post-mortem examination shows throughout the entire gastro-intestinal tract dark-colored patches of corrosion, and occasionally perforations.

Antidotes.-Alkaline carbonates or bicarbonates, or chalk to neutralize the acid; stomach-pump when practicable; oil, albumen, or milk to protect the mucous membranes; stimulants, opium, ammonia intravenously to counteract the depression of the vital powers.

TheraPeUtics. - The mineral acids are used in chronic diarrhoea and dysentery, the sulphuric acid combined with laudanum given in starch, gruel, or mucilage. Influenza-especially the sulphuric with quinine sulphate. Equine purpura - the sulphuric with iron sulphate. Hemorrhages, especially at parts remote from the stomach-the sulphuric with 
or without ergot. Irritable or ulcerated sore throat - either the sulphuric or hydrochloric, well diluted, as a drink. Atonic dyspepsia-hydrochloric with or without pepsin and bitters, given after feeding, except when there is acidity of the stomach. Acidity of the stomach-hydrochloric or, phosphoric in small doses before feeding. Torpidity of liver, chronic hepatitis-hydrochloric, nitric, or best of all, nitro-hydrochloric. Fevers-sulphuric with magnesium sulphate; for typhoid cases the hydrochloric is preferable. Intermittent and remittent fevers-nitric acid in full doses. Lead-poisoning - the sulphuric, to form the insoluble lead sulphate. General debility, convalescence - the sulphuric, with iron sulphate and nux vomica.

LOCAL UsES. - For ulcers, nitric acid as an escharotic. Also to destroy warts, fungous and malignant growths which cannot be otherwise conveniently removed. As a wash, well diluted, in Mallender's and chronic eczema. Irregular, sinuous, and poisoned wounds, the sulphuric acid or the black oil (sulphuric acid 1, spirit of turpentine 4, linseed oil 16), which is a convenient application to contract and remove umbilical hernia.

Foot-rot in sheep-the hydrochloric acid. A powerful astringent and styptic-Warren's styptic (sulphuric acid 5, spirit of turpentine 4, alcohol 4).

\section{Oils and Fats.}

The fixed oils and fats principally used in veterinary practice are: Adeps-lard; Oleum Morrhuce- 
cod-liver oil; Oleum Adipis-lard oil; Oleum Gossypii Seminis-cotton-seed oil; Oleum Oliva-olive oil; Lanolinum-lanolin, sheep's wool fat; Oleum Theobromoe-cacao butter.

Oleum Tiglii-croton oil; Oleum Ricini-castor oil; Oleum Lini-linseed oil. These three, being used mostly for their cathartic qualities, are classed with the evacuents.

Composition: Excepting cod-liver oil, all of the oils contain olein, stearin, and margarin in varying proportions-the olein giving fluidity, the stearin solidity.

Cod-Liver Oil is obtained from the fresh liver of the Gadus morrhua and other species of Gadus. It contains olein and margarin, gaduin (a principle peculiar to itself), propylamin, bile constituents, and traces of sulphuric and phosphoric acids, bromine, iodine, phosphorus, iron, lime, and magnesia. Three varieties are found in the market, of which the pale or white oil is the best, the two other darker varieties being of questionable utility.

Linseed Oil contains a large proportion of vegetable albumin, which coagulates on exposure to the air, thus making it a drying oil. This property is increased by boiling the oil.

Lanolin is a cholesterin fat obtained from the washings of sheep's wool, and contains from twentyfive to thirty per cent. of water. It has a faint odor, and differs from other fats in that cholesterin takes the place of glycerin; hence it does not saponify with an alkali. It is not liable to become 
rancid, unites readily with more than its own weight of water, is perfectly neutral as a base, readily penetrates the integument, carrying with it any medicament with which it is charged.

Cotton-seed Oil is a bland oil expressed from the cotton seeds, and largely takes the place of olive oil, which is usually adulterated with it.

Physiological Action.-Small quantities of fats and oils are said to be necessary for the digestion of nitrogenous foods, and to form the molecular basis of the chyle. Fat, which is an essential constituent of the products of tissue formation, is the main factor concerned in the production of force, and is prepared for assimilation by the action of the pancreatic juice and the bile. After oxidation fat is excreted as carbonic acid and water.

Therapeutics.-Olive oil, cotton-seed oil, and lard oil are nutrient, laxative, and emollient. The first two are occasionally given in doses of from onehalf to two pints to horses and cattle as a cathartic; for the same purpose in the dog from two to three fluidounces. They are also used to form the body of many liniments, i.e., as "Hartshorn liniment" (aqua ammonia 30, cotton-seed oil 70 ). The fats and oils are applied locally by inunction in chronic wasting diseases and in scaly skin diseases. In scrofulous conditions cod-liver oil is given internally, and also externally by inunction. Dose: H., fl. 弓 i.-ij.; C., fl. 3 ij.-iv.; D., fl. 3 i.-iv. It is very serviceable as an aid in convalescence from catarrh, influenza, or other debilitating disease, and may be advanta- 
geously given in broken wind in the horse, in chronic rheumatic disorders, chorea, and epilepsy. A very convenient preparation, when the pure oil is undesirable, is made by shaking together in a bottle equal volumes of cod-liver oil and lime water. For chronic rough in the dog this last preparation two ounces, and one ounce syrup of wild cherry bark; give one to two teaspoonfuls three to four times daily.

\section{Ferrum-Iron.}

Iron and its salts were the first mineral substances used in medicine-now about three thousand years ago-its chief function in the animal economy as well as in nature being that of an oxygen carrier. Iron is a normal constituent of the blood (1 part in 230 of red corpuscles) and tissues, where it exists as an oxide in combination with the hematin of the blood, and cannot exist in the blood without this. It is also present in bile, lymph, chyle, in the pigment of the eye, and in the gastric juice. Preparations of iron should be given in small doses, as but a small quantity is absorbed, and the rest is eliminated with the fæces, which are blackened as the result of the iron being transformed into a sulphide during its passage through the intestinal canal.

\section{Chief Preparations and Their Doses.}

Ferrum Reductum-iron reduced by hydrogen. This is a fine, grayish-black, lustreless powder, permanent in air, odorless, tasteless, and insoluble in water or alcohol. Dose: H., 3 ij.-iv.; D., gr. i.-v. 
Ferri Carbonas Saccharatus-saccharated carbonate of iron. A greenish-gray powder, gradually oxidized by contact with air, having at first a sweetish taste, and a metallic or ferruginous after-taste. Neutral reaction, and but partly soluble in water; completely soluble in dilute hydrochloric acid, with evolution of $\mathrm{H}_{2} \mathrm{CO}_{3}$, forming a clear yellow liquid. It is prepared by mixing freshly prepared carbonate of iron with sugar, and should contain about thirtyfive per cent. of the carbonate. Dose: H., 3 i.-iv.; D., gr. ij. $-\mathrm{x}$.

Ferri Iodidum-iodide of iron. Greenish tabular crystals, which turn brownish-red on exposure to air. As it is very readily decomposed it is but seldom used except as the more stable compound.

Syrupus Ferri lodidi-syrup of iodide of iron. This when properly prepared is a light green, clear syrup, and may be made extemporaneously as follows:

$$
\begin{aligned}
& \text { R Iodi puri., . . . . . } 3 \times 3 \text { iij.- } 9 \mathbf{i} \text {. } \\
& \text { Ferri pulv., . . . . . } 3 \text { vijss. } \\
& \text { Aquæ dest., . . . . . } \xi_{3} \text { iij. } \\
& \text { Syr. simplicis, . . . . } 3 \text { xxix. }
\end{aligned}
$$

Ft. syr. lege artis. Dose : H. fl. zss.-ij. ; D., $\Pi$ x.-xxx.

This should be kept in the light, in small bottles, and a small iron nail placed in each to take up any iodine which may become free from time to time.

Ferri Sulphas-green vitriol, copperas, sulphate of iron. Large pale-greenish prisms or lumps, efflorescent and absorbing oxygen on exposure to air, odorless, saline, styptic taste, acid reaction, soluble in 1.8 
parts of water, and insoluble in alcohol. Dose: H., 3 i.-iij.; D., gr. i.-iij. (Best given in aqueous solution as it is very irritant and astringent.)

Ferri Sulphas Exsiccatus-dried sulphate of iron, is the preceding salt deprived of its water of crystallization. The same in action, but the dose is only half of that given of the other.

Ferri Perchloridum-perchloride of iron. Orangeyellow crystalline pieces, very deliquescent, odorless or but slight odor of hydrochloric acid, strongly styptic taste, and acid reaction. Freely soluble in water, alcohol, and ether.

Liquor Ferri Perchloridi-solution of perchloride of iron, an aqueous solution containing thirty-eight per cent. of the salt. Dose: H., fl. 3 ss.-i.; D., m i. $-\nabla$.

Tinctura Ferri Chloridi-tincture of chloride of iron (liquor 35, alcohol 65). Dose: H., fl. 3 ij. $-\xi \mathrm{i}$.; D., $\pi \mathrm{v} .-\mathrm{xx}$.

Gossypium Hoemostaticum-styptic cotton, mado by dipping absorbent cotton in water, squeezing out, then dipping it in a solution of chloride of iron, again squeezing out superfluous liquid and drying.

Medicinal Actions of the Iron Preparations.

Iron carbonate is a mild chalybeate, especially used in canine practice, and is employed in the same diseases as the sulphate.

Iron sulphate is a restorative tonic, and in larger doses astringent and irritant. Externally-stimu- 
lant, astringent, styptic, and antiseptic. Its irritant action is counteracted by galls, alkaline carbonates, and demulcents. It is given in anæmia, dysentery, diarrhœa, scrofula, relaxed mucous membranes, wasteful serous or bloody discharges, as diabetes, red water in cattle, purpura, and various forms of hemorrhages. It antagonizes enlargement of spleen and liver, and has been given with good effect in chorea and epilepsy; also in convalescence from debilitating diseases. It should not be given if the bowel is very irritable, and should not be continued for more than two weeks at a time, allowing a few days' interval without its administration before resuming. Give soon after feeding-never on empty stomach.

Iodide of iron is a good tonic, alterative, and astringent, and may be used the same as the sulphate, but is especially effective in polyuria, nasal gleet accompanied by debility, and scrofulous and rheumatic swellings of the joints.

Iron chloride is used in red water in cattle, farcy, purpura, and typhoid complaints; distemper, loss of appetite from want of tone, hemorrhage in alimentary canal, and as an astringent and stimulant for the genito-urinary mucous membranes. In erysipelas halfounce doses of the tincture of iron have given most gratifying results. In sore throat give with chlorate of potash or muriate of ammonia. Diluted with water and injected per rectum to destroy ascarides. In the treatment of rheumatism in weak, debilitated animals, alternate it with salicylic acid. Externally it is applied as antiseptic, astringent, and styptic. 
Massa Ferri Carbonatis-Vallet's mass, is a very suitable preparation for the smaller domesticated animals. Dose for dog, gr. i.-v. It is prepared by beating into a mass: Ferrous sulphate 100, sodium carbonate 110 , honey 36 , sugar 25 , syrup and water a sufficient quantity to give proper consistence.

\section{Bismuthum-Bismuth.}

Chief Preparations.-Bismuthi Subcarbonasbismuth subcarbonate; Bismuthi Subnitras-bismuth subnitrate. Doses: H., 3 i.-iij.; D., gr. ij.-x. These salts of bismuth are heavy white powders, insoluble in water or alcohol, and nearly so in the gastric juice. They act as local sedatives and astringents to the stomach and intestines, relieving irritation in dyspepsia and vomiting. They are frequently given in diarrhœa and dysentery, combined with tannin, opium, or catechu.

Bismuthi Subiodidum-bismuth subiodide, a heavy, dark-red, insoluble powder, with very powerful antiseptic and stimulant properties, is used only externally as a dressing for wounds, ulcers, etc.

\section{Arsenium or Arsenicum-Arsenic.}

Chief Preparations. - Acidum Arseniosum, Arsenious Acid, White Arsenic-a dull white powder, specific gravity 3.8, colorless, tasteless; sprinkled on red-hot coals it gives off a garlicky odor; completely volatilizes at $400^{\circ} \mathrm{F}$. Sparingly soluble in alcohol and water $(1: 33)$, but its solubility is in- 
creased by acids and alkalies. Dose: H., gr. v.-x. ; D., gr. $\frac{1}{15}-\frac{1}{10}$, after feeding.

Liquor Potassii Arsenitis-solution of potassium arsenite, Fowler's solution, contains one per cent. of arsenious acid. It is prepared by heating in a flask: Potassium carbonate and arsenious acid, of each gr. lxxxvij; distilled water, Oi. Dose: H., fl. $\zeta$ ss.-i.; D., $\pi$ ij.-xv., after feeding.

Physiological Action.-Arsenic is a powerful escharotic and excites violent inflammation locally; given internally, it is a powerful irritant to the gastro-intestinal and bronchial mucous membranes, and should always be given after feeding. Small doses act as a stomachic tonic, promoting the appetite and digestion, increase cardiac action, respiratory power, and the secretions of the intestinal canals; also stimulate intestinal peristalsis and increase the sexual appetite. Full medicinal doses, if continued, cause œdema and itching of the eyelids, increased saliva, nausea, vomiting of mucus, diarrhœa or dysentery, enfeebled heart, soreness to the touch over epigastric region, itchy skin with small eruptions, jaundice, and albuminuria. Toxic doses produce colicky pains, vomiting, great thirst, dysentery, strangury, suppressed or bloody urine, rapid feeble heart, cold breath, and collapse. Post-mortem examination shows èchymotic spots, erosions, softening of the gastro-intestinal mucous membranes, fatty degeneration of internal organs, especially kidney and liver; also congestion of lungs and bronchial mucous membranes. 
Treatment of Arsenical Poisoning.-The best antidote is the freshly-prepared hydrated oxide of iron* in the proportion of eight parts for every one part of the poison. When practicable, emetics or stomachpump; cathartics may also be given; oil, milk, mucilaginous drinks, to protect the mucous membranes. Further treatment must necessarily be symptomatic.

Therapeutics.-As a gastric tonic in dyspepsia and gastralgia. In chronic catarrhal diarrhœa, when partly digested food is passed. Chronic bronchial and pulmonary affections. As an alterative in the early stages of tuberculosis and farcy. In rheumatism, chorea, epilepsy, chronic eczema, scab, and mange. In periodic fevers, combined with quinine; in weak heart with stocking of legs, give with iron and nux vomica or strychnine. Also in chronic scaly skin diseases. Externally to eradicate warts, slough out fistulæ and tumors. As a sheep dip-arsenic $2 \frac{1}{2}$ pounds, sodium carbonate 3 pounds, soft soap and sulphur, of each 5 pounds, water 100 gallons. Use with care!

\section{Bitter Tonics.}

The chief members of this group are Gentian and Cinchona.

Gentiana-gentian, the dried root of Gentiana

* To prepare hydrated oxide of iron.-To a solution of sulphate or chloride of iron add water of ammonia till a precipitate is no longer formed; collect this precipitate on a cloth and wash till ammonia is removed; mix with water and give at once. 
lutea, a perennial European plant. Gentian is a simple bitter, improves the appetite and general tone, and aids digestion. It is used as a tonic and stomachic in all veterinary patients. Its chief preparations are: Pulvis gentianæ, powdered gentian root: H., 3 ss.-i.; D., gr. x.-xxx. Extractum gentianæ fluidum, fluid extract of gentian: H., fl. 3 ss.-i. ; D., Tl x.-xxx. Tinctura gentianæ composita, compound tincture of gentian: Prepared from cardamom 2, bitter orange-peel 4, gentian 8, diluted alcohol enough to make 100 parts. This last preparation is used mostly in canine practice. Dose: H., fl. 3 i.-iij.; D., fl. 3 ss. - ij.

Cinchona, Peruvian bark. There are three chief varieties of cinchona, viz. :

1. Cinchona, the bark of any species of cinchona containing at least three per cent. of alkaloids.

2. Cinchona Flava, yellow bark or calisaya bark, obtained from the cinchona calisaya, and containing the most quinine.

3. Cinchona Rubra, red bark, obtained from the cinchona succirubra, and containing about equal parts of quinine and cinchonine.

Another variety, the Cinchona Pallida, or pale bark, contains the most cinchonine, but is not of so much importance as the others. The cinchona tree is a native of the mountainous parts of western South America, but is also planted and raised for bark in India, Burmah, Ceylon, and various other suitable places.

Composition of Cinchona.-It contains four chief 
alkaloids, called: Quinina, quinine-the most valuable. Quinidina, quinidine-the strongest antiperiodic. Cinchonia, cinchonine-the least valuable. Cinchonidina, cinchonidine-about half the strength of quinine. Besides these are found seventeen other alkaloids of minor importance, two simple acids, two tannic acids, one resinoid, and coloring matter.

Chinoidin is the black bitter residue left after the crystallizable alkaloids have been removed from the mother liquor, and evaporated. It is one-fourth as strong as quinine.

Quinine is prepared by boiling the coarsely ground bark in dilute hydrochloric acid, and treating the filtered solution with lime until it is alkaline in reaction. This process precipitates the alkaloids and the coloring matter. The collected precipitate is then boiled with alcohol, which dissolves the quinine and cinchonine. This last solution is neutralized with sulphuric acid, boiled with animal charcoal, and filtered. This filtered liquid, on standing, allows the quinine sulphate to crystallize out, and retains the cinchonine in solution. Quinine sulphate occurs in delicate feathery or silky whitish prisms, has an intensely bitter taste, is soluble in seven bundred and forty to nine hundred parts of cold water, readily in alcohol, soluble ether, chloroform, and dilute acids.

Chief Preparations of Cinchona employed in veterinary practice are:

Pulvis Corticis Cinchonoe, powdered cinchona bark. Dose: H., 3 ij.-iv.; D., gr. xx.-xl. 
Tinctura Cinchonce Composita, compound tincture of cinchona bark, U. S. P. (Red bark 10, serpentaria 2, orange peel 8 , glycerin 10 , alcohol and water, of each a sufficient quantity to make 100 parts.) Dose: H., fl. żi.-iij.; D., fl. 3 ss.-iv.

Infusum Cinchonce, infusion of cinchona. (Cinchona 6, aromatic sulphuric acid 1, water 93.) Dose: H., Oss.-i.; D., fl. 3 i.-iv.

Quinince Sulphas, sulphate of quinine. Dose: H., gr. xv.-3i.; D., gr. i.-v.

Cinchonidince Sulphas, sulphate of cinchonidine. Dose: H., 3 ss.-ij. ; D., gr. ij.-x.

Quinince Bimuriatis Carbamas, the double hydrochlorate of quinine and urea, a very solubie salt, is especially adapted for hypodermic medication. Dose for dog, inject grs. $1 \frac{1}{2}$ to 8 dissolved in $\pi$ lo to 20 of distilled water; dose for horse, inject grs. 15 to 30 dissolved in $\mathrm{fl} .3 \mathrm{ss}$. to ij. of distilled water.

Other quinine salts, as the muriate and bisulphate, are given in the same doses as the sulphate.

Incompatibles are free tannic acid, iodine, alkalies, and alkaline carbonates.

Physiological ACtion.-Cinchona bark is an astringent bitter tonic, antiseptic, antiperiodic, antiphlogistic, antipyretic, and emmenagogue. It diminishes reflex action, acts as a protoplasmic poison, and its alkaloids, while rapidly diffused, are but slowly eliminated, being found in the urine in fifteen minutes after taking, and as late as three days after; the maximum point of action being reached in about four to five hours after its first administration. Qui- 
nine is held in solution in the blood by carbonic acid. When first given it promotes the appetite, improves digestion, stimulates the flow of saliva and gastric juice; but if very long continued brings on gastric catarrh, indigestion, and constipation. Small doses stimulate the heart, while large doses depress the heart and enfeeble the pulse. Moderate doses cause hyperæmia of the brain, and large doses produce anæmia of the brain. Cinchonism is the term applied to the effects of continued large doses, i.e., headache, amaurosis, deafness, staggering gait, irregular heart's action, convulsions, etc. The spleen is reduced in size and the temperature of pyrexia lowered, but it does not reduce the body temperature of a healthy animal. It is a uterine stimulant in labor, and lessens the excretion of uric acid, but not that of urea. It is used in malarial and intermittent fevers, acute catarrh, surgical fever, and erysipelas (with iron and arsenic); as a tonic in indigestion and general debility with nux vomica; in asthma and diarrhœa. Also in diabetes, influenza, strangles, and purpura. A five-per-cent. solution of the muriate of quinine is frequently used to wash out the bladder in cystitis. Quinine should not be given in inflammatory diseases of the bowel if accompanied by constipation and irritability of the stomach. Quinine may be given in bolus or solution, and its action is said to be increased by conjoining with capsicum or ginger.

Ferri et Quinince Citras, or Ferro-Chininum Citricum, the citrate of iron and quinine, a double salt, used mostly in canine practice, is given in pill 
or aqueous solution. Dose for dog: gr. ss-ij. This combines the actions of the two, iron and quinine, and is a very valuable tonic.

\section{AGENTS PROMOTING WASTE.}

\section{Potassium.}

Potassium is one of the metallic elements. Symbol, K; atomic weight, 39 ; quantivalence, 1 . It has great affinity for oxygen, and decomposes water with considerable violence, replacing one or both of its hydrogen atoms. It is obtained as "carnallite," a chloride of potassium and magnesium, in or near rock-salt beds; also from wood ashes.

Chief Preparations.-Potassa, caustic potash, hydrated protoxide of potash. This is obtained by dissolving the ashes of plants in water (when calcined these lose their organic impurities); this then is pearl ash or impure potassium carbonate. This solution is boiled with calcium hydrate, forming calcium carbonate as a precipitate, and leaving potassium hydrate in solution.

Liquor Potassce, solution of potassa (Potassa $\zeta$ i., dissolved in distilled water Oi.). Potassa and its solution are powerful caustics, and are rarely used internally.

Potassii Acetas, potassium acetate, a white, soluble, deliquescent salt, obtained by neutralizing potassium carbonate with acetic acid. Dose: H., z ss.-i. ; D., gr. v. $-\mathrm{xv}$. 
(A) Potassii Carbonas, potassium carbonate. (B) Potassii Bicarbonas, potassium bicarbonate. Dose of either of these for H., 3 ss.-i.; D., gr. $\mathrm{x} .-\mathrm{xxx}$.

(A) is prepared from wood ashes or by burning potassium tartrate with charcoal.

$(B)$ is prepared by passing carbonic-acid gas through a strong aqueous solution of the neutral carbonate. Occurs in transparent, colorless, rhombic prisms, soluble in four times its own weight of water.

Potassii Nitras, potassium nitrate, nitre, saltpetre. Dose: As febrifuge, H., 3 ij.-iv.; D., gr. v. $-\mathrm{xv}$. As diuretic, H., 3 ss. $-\mathrm{i}$.; D., gr. x. $-\mathrm{xxx}$. Occurs as whitish, opaque, crystalline masses, or transparent, colorless six-sided prisms, having a sharp, cooling, saline taste, permanent in air, and soluble in three and one-half parts of cold water.

Potassii Chloras, potassium chlorate. Dose: H., 3 i.-iv.; D., gr. v. $-\mathrm{xv}$. Occurs as colorless rhomboidal plates, having a saline cooling taste, and soluble in sixteen parts of cold water; prepared by rapidly passing chlorine gas through a strong solution of potassium carbonate and calcium hydrate.

Potassii Permanganas, potassium permanganate. Small, dark-brown, needle-shaped or prismatic crystals, soluble in sixteen parts of cold water, making a deep-red solution.

Potassii Iodidum.-See under "Iodides."

Potassii Bromidum.-See under "Bromides." 
Physiological Action of Potassium and Its SALTS.-Caustic potash dissolves albumin, saponifies fats and destroys tissues, by combining with their water. The potassium salts promote tissue waste; small doses promote the formation of gastric juice if given on an empty stomach, but large doses act chemically and retard digestion by neutralizing the free acids.

The carbonate and bicarbonate, if given on an empty stomach, enter the blood unchanged, combine with the neutral phosphate of sodium contained therein, converting it into an acid phosphate, so that when eliminated by the kidneys the urine is more acid. If, however, they have been given on a full stomach, they are decomposed before entering the blood, and the urine is therefore now more alkaline.

The acetate is absorbed in its own form, is decomposed in the blood, makes the blood and urine more alkaline, and acts as a diuretic, increasing both the water as well as the solids of the urine, but decreasing the amount of uric acid by increasing oxidation. The nitrate and chlorate are absorbed and eliminated in their own form, the nitrate being a powerful diuretic, febrifuge, and alterative; externally applied, it is stimulant and refrigerant. The chlorate is antiseptic, alterative, sialagogue, and diuretic; externally, antiseptic, refrigerant, and mildly stimulant. Large doses of the chlorate are very irritant to the kidneys, and may produce albuminuria. All the potassium salts in large doses are cardiac poisons, mus- 
cle paralyzers, poisonous to protoplasm, and hinder the ozonizing function of the blood. The permanganate is antiseptic, deodorant, and mildly astringent.

Poisoning by Caustic Alkalies.-Give rinegar or acetic acid; then demulcents and oils to protect the mucous membranes; support the vital powers.

TheraPeUtiCs.-Liquor potassæ as a caustic to eradicate warts, fungous growths, to poisoned wounds. Its excessive action may be rapidly checked by bathing the part with dilute acetic acid. It should be used only with great care.

Rheumatism - the carbonate, bicarbonate, and nitrate, to saturate the blood and render the urine more alkaline.

Acidity of stomach and atonic dyspepsia-the carbonate or bicarbonate, before feeding.

Ulcerative stomatitis, aphtha-the chlorate as a wash; also in catarrhal affections; in follicular pharyngitis, sore throat, and diphtheritic affections, combined with tinctura ferri chloridi.

The carbonate and bicarbonate are used for the relief of phosphatic deposits and calculi in dogs. Externally as stimulants and to soften and remove thickened skin; to relieve the itching of certain skin diseases. The carbonate is usually employed as a wash before giving treatment for mange.

The nitrate promotes bronchial, cutaneous, and urinary secretions; it is an excellent febrifuge and diuretic. Frequently used externally with ammonia muriate, alcohol, and water as a cooling lotion. A fa- 
vorite diuretic bolus is composed of potassium nitrate 3.s., soap 3 i., resin 3 ss., Venice turpentine 3 ss. For sore throat a bolus of the nitrate $\xi$ ss., extract of belladonna 3 ss., eamphor 3 i., or the same made up into an electuary with powdered slippery elm bark and syrup. This last has been found serviceable in bronchitis, pharyngitis, and laryngitis. Together with tincture of aconite the nitrate is considered the best treatment for Laminitis by many practitioners. The acetate produces slight diuresis, helps to reduce temperature, alkalinifies the blood and urine, is given in inflammations to promote the excretion of resulting products, also in irritability of the kidneys and bladder.

The permanganate destroys bacteria, is used in solution ( 3 i.-iij. to aqua Oi.) as a disinfectant and deodorant wash for foul wounds, sores; for washing the hands after dissections and post-mortem, etc., in weak solution. It has been found to be the best antidote for morphine or opium poisoning. Doses of from gr. $\mathrm{x}$. to xxx. are recommended for the horse in septicæmia.

\section{SodIum.}

Sodium occurs in combination naturally in large quantity in sea water, marine plants, etc.; also as rock salt, as borax, and in the Chili nitre beds.

Chief Preparations.-Sodii Carbonas, sodium carbonate, "washing soda." Sodii Bicarbonas, sodium bicarbonate, "baking soda," "soda salæratus." Sodii Chloridum, sodium chloride, common salt. 
Sodii Boras, borate or biborate of sodium, borax. Sodii Sulphas-sodium sulphate, Glauber's salt. For sodium sulphite and sodium hyposulphite, see under Sulphur and Sulphites.

The physiological actions of the sodium salts are similar to those of potassium salts, but they are not so alkaline, not so depressant, nor are they such powerful cardiac and nerve poisons as the potassium salts.

The Sodium Carbonate and Bicarbonate are antacids and alteratives. Dose: H., 3 i.-iv.; D., gr. v. - $\mathrm{xxx}$.

Sodium Chloride.-Small doses are restorative, stomachic, and antiseptic; larger doses irritant, cathartic, and emetic; externally stimulant, refrigerant, and antiseptic. Dose: H., lb. ss.-i. (on this animal its action as a purgative is uncertain and irritant); cattle, lb. ss.-i.; sheep, 亏 i.-iij. (is more adapted for these animals than for the horse).

Sodium Borate.-Antiseptic, parasiticide, slightly astringent, and alkaline. Dose same as that of the bicarbonate.

Glauber's Salt.-Cathartic, cholagogue, febrifuge, and slightly diuretic. Has very low diffusive power, impedes absorption of fluids in the intestinal canal, but increases the amount of intestinal secretion and stimulates peristalsis; produces an increase in the quantity of bile secreted by its direct action upon the hepatic cells. As a cathartic it is slow in operation, generally requiring from twenty to twenty-four hours, and is more suitable for cattle than for horses. 
Dose: H., lb. ss.-i. ; cattle, lb. ss.-iss. It is usually combined with ginger, capsicum, or other stomachics.

Thereapeutics of the Carbonates.-Small doses increase secretion of gastric juice, and are used in indigestion, flatulence, acidity of the stomach, atonic dyspepsia, and rheumatism. A solution of the bicarbonate is applied frequently to burns, as an injection in simple leucorrhœa, and to relieve the irritation of urticaria and other skin eruptions.

Therapeutics of THE Borate.-Ulcerative conditions of the mouth, aphtha. To relieve the irritation of eczema and other skin diseases it is applied locally in aqueous solution. A weak solution is used to dissolve the gummy substance from the eyelids in purulent conjunctivitis; also as an eye lotion. Combined with thymol, carbolic acid, and eucalyptol as a spray or gargle in ulcerated sore throat and catarrhal conditions of the respiratory mucous membranes. The appended formula of Dr. Carl Seiler's solution, which is a similar preparation, can be obtained in the form of compressed tablets:

R. Sodium bicarbonate,

Sodium biborate,

. $\bar{a} \mathbf{a} 3 \mathrm{ij}$.

Sodium benzoate,

Sodium salicylate, . . . . . . $\bar{a} \bar{a}$ gr. $\mathbf{i j}$.

Eucalyptol, menthol, and thymol, . . āā gr. i.

Oil of wintergreen, . . . . gtt. ij.

Alcohol, . . . . . . . $3 \mathrm{ij}$.

Glycerine, . . . . . . 3 iiss.

Distilled wate, . . enough to make Oi. 
Therapeutics of THE Chloride.-As a purgative for cattle and sheep, small repeated doses are stomachic; given together with gentian and ginger in indigestion and irregular appetite; injections of two or three ounces to one pint of water per rectum to carry away ascarides. A five- to ten-per-cent. solution as an antiseptic and stimulant gargle in relaxed and ulcerated sore throat. Salt-water baths are stimulant to the skin. An excellent cooling lotion may be prepared from salt, saltpetre, and muriate of ammonia, of each two ounces, dissolved in one quart of water. As an emetic for the dog, one to four drachms dissolved in warm water.

\section{Calcium.}

Calcium is one of the alkaline earths. Carbonate of lime or chalk is the source from which most of the calcium salts are prepared. Calcium oxide, calx, lime, quicklime, $\mathrm{CaO}$, prepared by burning limestone, marble, chalk, or other form of calcium carbonate, is a powerful irritant, and in its own form is but seldom given internally.

Chief Preparations. - Liquor Calcis, Aqua Calcis, lime water. Dose: H., fl. żiv.-v.; D., fl. 3 i.-iv.

Calcii Carbonas Procipitatus, precipitated chalk, calcium carbonate. Dose: H., 亏 i.-ij.; D., gr. $\mathrm{v} . \mathrm{-xx}$.

Calcii Phosphas Proecipitatus, precipitated phosphate of calcium. Dose: H,, 3 ij.-iv.; D., gr. i. $-\mathrm{x}$. 
Calx Chlorata, chlorinated lime, or bleaching powder (misnamed chloride of lime). Dose: H., 3 i.-ij.; D., gr. ij.-v., in cold gruel or water.

For Calx Sulphurata, see under Sulphur.

Physiological ACtion and Therapeutics.The calcium salts and preparations are feebly alkaline, astringent, and antacid. The liquor calcis is irritant, astringent by contact, antacid, and sedative, and is given in indigestion and diarrhœea of all patients, especially in young animals when there is a tendency to acidity of the stomach. As an antidote for poisoning by the mineral acids, by carbolic acid, and by oxalic acid. In bronchial filariæ in calves and sheep, sometimes combined with turpentine. Enemata of aqua calcis to destroy ascarides. A most excellent application to scalds and burns is "carron oil" (equal volumes of liquor calcis and linseed oil). A spray of aqua calcis has been found serviceable in diphtheria and croup. Lime in solution, i.e., as "whitewash," is used for deodorizing and cleansing stables, etc. It is customary to add carbolic acid or other disinfectant to whitewash to render it more effective for this purpose. Chalk, which is the usual cause of hardness of water, is used for the same purposes as the aqua calcis. The calcium phosphate, which is an essential ingredient of all animal tissues, combines the actions of calcium and phosphorus, and is essentially a restorative; it increases the alkalinity of the blood, increases the power of that tissue to hold $\mathrm{CO}_{2}$, diminishes the excretion of urea, and is very serviceable in chronic diarrhœa, anæmia, and 
other forms of malnutrition, especially in young animals. It is also employed in caries, protracted suppuration, osteomalacia, rachitis, and scrofular disorders. Chlorinated lime, which is a powerful bleaching agent, is irritant, stimulant, astringent, antiseptic, and alterative; it is used mostly externally. Toxic doses produce local irritation, difficult breathing, acceleration of the pulse, weeping eyes, an abundant secretion of urine having a white sediment and a slight odor of chlorine, and in dogs vomiting.

Externally it is used as a stimulant, antiseptic, deodorant, disinfectant, and parasiticide. Has been found serviceable by some practitioners in tympanites in horses, hoven in cattle, is applied externally to unhealthy wounds, for destroying the fungus of ringworm, and the parasites of mange and grease. As a disinfectant and deodorizer for stables, etc., it is very efficient.

\section{Ammonium.}

Liquor Ammonice fortior, Aqua Ammonice fortius, concentrated ammonia, caustic ammonia, stronger water of ammonia. Dose: H., fl. 3 i.-ij.; D., $\Pi$ v. -x. (should be given well diluted). A colorless liquid, pungent, caustic, of a very strong suffocating odor; strong alkaline reaction; specific gravity, 0.891. Contains about twenty-eight per cent. of gaseous ammonia $\left(\mathrm{NH}_{3}\right)$ in solution.

Liquor Ammonice, Aqua Ammonice, water of ammonia, Hartshorn spirit. A diluted solution of the concentrated ammonia, containing about ten per 
cent. of gaseous ammonia. Has specific gravity 0.959 , and is prepared by mixing one part of concentrated ammonia with two of water, by volume. Dose: H., fl. 3 iij.-vi.; D., Tl xv.-xxx.

Spiritus Ammonii Aromaticus, aromatic spirit of ammonia (ammonium carbonate; water of ammonia; oils of lemon, lavender, pimento; alcohol and water). Dose: H., fl. 3 ij.-viij.; D., ml x.-xxx.

Liquor Ammonii Acetatis, solution of acetate of ammonia, Mindererus spirit (dilute acetic acid neutralized by addition of ammonium carbonate). Dose: H., fl. 3 ij.-iv.; D., fl. 3 ij.-iv.

Ammonii Carbonas, carbonate of ammonia, Hartshorn salt. Dose: H., 3 ij.-iv.; D., gr. iij.viij.

Ammonii Murias, Ammonii Chloridum, muriate or chloride of ammonium, sal ammoniac. Dose: H., 3 ij.-iv.; D., gr. iij.-viij.

Linimentum Ammonia, ammonia liniment, Hartshorn liniment (aqua ammoniæ 30, cottonseed oil $\%$ ).

For Ammonium Bromide, see under Bromides.

Physiological Action.-Liquor ammoniæ if used locally causes considerable irritation, abstracts water from the tissues, dissolves the epithelial scales, liquefies their albumin, and saponifies their fats-therefore acting as a caustic. Full doses given internally stimulate the spinal cord, motor nerves, and muscles, are said to produce tetanic convulsions, and finally paralyze the cord. Suffocation may be brought about by large quantities of the gas en- 
tering the air passages. Large concentrated doses given internally will cause violent gastro-enteritis and paralysis of the brain centres from absorption. Medicinal doses stimulate the vasomotor centre by reflex action and raise blood pressure. After absorption there is direct stimulation of the respiratory and circulatory nerve centres and promotion of the secretions of skin and mucous surfaces. It is given internally as an antacid, a diffusible stimulant, antispasmodic, and stimulant expectorant; externally as a stimulant, counter-irritant, and vesicant.

Antidotes for poisoning by liquor ammoniæ are vinegar and dilute acids with diluents and demulcents.

Spiritus ammoniæ aromaticus conjoins the properties of its constituents (ammonium carbonate and water of ammonia).

Ammonium carbonate is more permanent in its effects than the liquor ammoniæ, but not quite so powerful. It is a good antacid, and diffusible stimulant; small doses promote secretion of gastric juice, larger doses relieve flatulence and spasm. It is a good cardiac and respiratory stimulant, and promotes bronchial secretion and expectoration. Toxic doses are irritant, cause primary stimulation and secondary paralysis of the cord and motor centres.

Ammonium chloride is a stimulant to the alimentary and respiratory mucous membranes in moderate doses, promoting their secretions. It has marked cholagogue powers and promotes excretion of urea. Externally it makes a very serviceable cooling lotion. 
Solution of acetate of ammonia is not so powerful in action as either the liquor or the carbonate, but is a good diaphoretic, antipyretic, a mild diuretic, and expectorant.

Therapeutics. - Liquor Ammonice: Indigestion, tympanites, spasmodic colic, in ruminants especially; influenza, pneumonia, bronchial disorders, poisoned bites and stings. Antidote against poisoning by opium, aconite, and digitalis. Externally as counter-irritant (ammonia liniment) to rheumatic swellings of joints and muscles; sore throat.

Ammonium Carbonate: Atonic dyspepsia of all animals, acidity of the stomach, to relieve flatulence and spasm; in influenza, scarlatina; in bronchial disorders and pneumonia, especially at crisis to liquefy the products of inflammation and counteract deficiency of vital powers, and whenever a prompt stimulant is required.

Ammonium Chloride: In bronchial and gastric catarrh in all animals, especially after the more acute symptoms have subsided; torpidity of the liver, catarrh of the bile ducts and its jaundice; duodenal and intestinal catarrhs; locally in solution to inflammations, bruises, and sprains.

\section{Vegetable Acids.}

Of these but one is used in veterinary practice, namely, the acetic.

Acidum Aceticum Purum, U. S. P., pure acetic acid, containing thirty-six per cent. absolute acetic 
acid, and sixty-four per cent. water. Dose: H., fl. 3 i. $-\mathrm{ij}$.; D., Tl ij.-v.

Acidum Aceticum Dilutum, dilute acetic acid, containing six per cent. absolute acid and ninetyfour per cent. water.

Acetum, vinegar, an impure dilute acetic acid, prepared by acetous fermentation of wine, cider, or other fruit juice.

Pure acetic acid is a colorless, strongly acid liquid, having a pungent odor, and is prepared by the destructive distillation of wood or sawdust; the condensed products separate into two layers-the lower one wood-tar; the upper, a two- to four-per-cent. solution of pyroligneous or impure acetic acid, together with wood alcohol, acetone, and other allied bodies. Sodium carbonate is now added, the volatile hydrocarbons distilled off, and the remaining liquid evaporated down-which leaves sodium acetate. This in turn is further decomposed by sulphuric acid, and again distilled, thus forming acetic acid. Acetic acid dissolves resins, camphor, volatile oils, and many alkaloids, and unites with bases to form acetates. Acetum or vinegar may also be prepared by the destructive distillation of wood, but usually is obtained from the oxidation of impure alcohols by exposing them to the air at about $80^{\circ} \mathrm{F}$. while in contact with a ferment. For this purpose the poorer grades of wine, also cider, are used.

Physiological Action.-Acetic acid is corrosive, irritant, and vesicant, and is seldom used internally, except in combination. Externally, diluted, 
usually in the form of vinegar, as a stimulant and refrigerant. It forms salts in the stomach, enters the blood, when it becomes oxidized, producing carbonic acid, increasing the acidity of the urine in its excretion, at the same time also increasing the amount of water in the urine. Secretion in general is promoted by small doses, but its long-continued use causes indigestion, emaciation, and poverty of the blood. Rubbed briskly into the skin it causes redness; if continued it forms blisters resembling those produced by scalding water. It is applied to remove warts, scurf, to destroy cryptogamic parasites and acari; well diluted in mallenders, sallenders, scab, and mange. The diluted acid or vinegar combined with salt or sal-ammoniac, to contusions, sprains; dilute vinegar for sponging the skin of febrile patients, and to check excessive perspiration. Also as a test for albumin in urine.

\section{Sulphur, Sulphites, SUlphides.}

Sulphur Lotum, U. S. P., washed sulphur; Sulphus Prcecipitatum, U. S. P., lac sulphur, milk of sulphur, precipitated sulphur. Both of these are preparations of sublimed sulphur. Dose: As laxative, H., 亏i.-iv.; C., 亏 iij.-vi.; D., 3 i.-iv.; as alterative, H., 3 ss.-ij.; C., 3 ij.-iv.; D., 3 ss.-ij.

Sodii Sulphis, sodium sulphite; Sodii Hyposulphis, sodium hyposulphite. Dose: H. and C., 亏s.-i.; D., gr. x.-xl.

Calx Sulphurata, sulphurated lime, so-called sul- 
phide of calcium. Dose: H. and C., gr. x.-xl.; D., gr. $\frac{1}{10}-\mathrm{ij}$.

Sulphur, commonly called "brimstone;" symbol, S; atomic weight, 32 ; quantivalence, 2,4 , and 6 ; is a non-metallic element, distinguished by its yellow color and crystalline properties. It is one of the acid elements, and unites with $\mathrm{O}$ to form the most powerful acid radicals. It is almost tasteless, insoluble in cold water or alcohol, but soluble in benzol, carbon bisulphide, fixed and volatile oils. It is completely volatilized by heat, burns with a pale blue flame, and gives off strong suffocating fumes of sulphurous anhydride while burning. It is found in many animal substances as sulphates, in many mineral waters as hydrogen sulphide, in certain volatile vegetable oils, in the pyrites or metallic sulphides; also as native sulphur in blue clay beds in Sicily and Italy. Sublimed sulphur is prepared by distilling the crude sulphur and allowing its vapor to condense as a fine yellow powder, in large chambers or receptacles built for that purpose.

Sulphur Lotum, a fine lemon-yellow powder, odorless and almost tasteless, insoluble in water or alcohol, is prepared by digesting for three days 12 parts of sublimed sulphur with a mixture of water 12 parts, and water of ammonia 1 part. After occasional agitation for three days the whole mixture is strained through muslin, and the residue washed with water until the liquid running from the strainer no longer produces a precipitate with test solution of chloride of barium. 
Sulphur Procipitatum, a very fine, yellowishwhite, amorphous powder, odorless and almost tasteless, insoluble in water or alcohol, is prepared by boiling together sublimed sulphur 100 parts, slaked lime 50 parts, water 1,450 parts, which forms calcium sulphide and hyposulphite. When the mixture has cooled it is filtered; to the filtered liquid is added a mixture of equal volumes of $\mathrm{HCl}$ and water until almost neutral, when the sulphur is thrown down as a fine precipitate, which after being thoroughly washed is dried by gentle heat.

Sodii Sulphis, transparent, colorless, monoclinic prisms, efflorescent in dry air, odorless, having a cooling, saline, and sulphurous taste and a neutral or feebly alkaline reaction; soluble in 4 parts cold water, in 0.9 part of boiling water, almost insoluble in alcohol. It is prepared by passing sulphurous acid gas through a solution of sodium carbonate, thus precipitating crystals of acid sulphite of sodium; this is further saturated with sodium carbonate and rendered alkaline in reaction.

Sodii Hyposulphis, large, transparent, colorless monoclinic prisms or plates, efflorescent in dry air, odorless, having a cooling, somewhat bitter and sulphurous taste, neutral or feebly alkaline reaction, insoluble in alcohol, soluble in 1.5 parts cold water, partly decomposed by boiling water. Must be kept in well-stoppered bottles. It is prepared by dissolving sulphur in a boiling solution of sodium sulphite and crystallizing.

Calx Sulphurata, a grayish-white powder, altered 
by exposure to air, having a foul, sulphurous odor, an offensive alkaline taste, alkaline reaction, slightly soluble in water, insoluble in alcohol, is prepared by exposing in a crucible for one hour at a low red heat a mixture of lime 100 parts and precipitated sulphur 90 parts. The finished product should contain not less than thirty-six per cent. of absolute calcium sulphide.

Physiological Action.--Sulphur is an alterative, a mild laxative, a stimulant to the intestinal mucous membranes, a disinfectant and deodorizer, especially in the form of sulphurous acid. Being soluble in alkaline solutions it is partly absorbed in the small intestine and stimulates its mucous membrane. It is excreted mainly by the fæces, but also by the urine as sulphates, by the skin and respiratory apparatus, and as it communicates a disagreeable odor and taste to the meat, must not be administered to animals used for food for some time prior to slaughtering. Sulphites, sulphides, and hyposulphites are in part decomposed by the acid secretion of the stomach, partly converted into sulphates, are absorbed and are eliminated chiefly by the kidneys. If their administration is continued for a longer period they impair digestion, impoverish the blood, cause anæmia and emaciation. The sulphites and hyposulphites have great power to diminish the effects of animal poisons, and are used as febrifuges, antiseptics, antacids, and carminatives.

THERAPEUTICS.-Sulphur as a laxative, especially during pregnancy and in convalescence from acute 
diseases; also in rheumatism and in lead-poisoning; as an ingredient of many alterative and condition powders; locally in eczema, scab, mange, and grease -as carbolized sulphur ointment. As a disinfectant and deodorizer it is burned to allow its vapor to saturate the air of stables, cowsheds, and kennels. Sulphites and hyposulphites-to relieve flatulence and acidity. in calves and foals, and in contagious pleuro-pneumonia in cattle as internal antiseptics. In distemper in dogs and cats; also in septicæmia and pyæmia of all animals; in foot-and-mouth disease in cattle.

Calcium sulphide, so-called, is used to prevent and limit pus formation. In crops of boils, scrofulous sores, glandular enlargements, or extensive suppuratinn it is very efficient, especially if given in small, repeated doses.

\section{ALTERATIVES.}

\section{IODINE AND IODIDES.}

Iodum, iodine, is a non-metallic element; symbol, I; atomic weight, 127; quantivalence, 1. It occurs in most marine plants, in sea water, cod-liver oil, also as mineral iodides and iodates. In its crude form it appears as bluish-black rhombic plates, having a metallic lustre, peculiar odor, acrid taste, and neutral reaction. Sparingly soluble in water, very soluble in ether, in 12 parts of alcohol, and in solutions of KI and salt. 
Preparations of Iodine.-Iodum, iodine (in bolus, but rarely given in this form). Dose: $H$. and C., gr. X.-xxx.; D., gr. ij.-vi.

Liquor Iodi Compositus, compound solution of iodine, Lugol's solution (always well diluted). Dose: H. and C., fld 3 ss-iv.; D., $\pi$ i.-x. (Composed of iodine 5, potassium iodide 10 , water 85 .)

Tinctura Iodi, tincture of iodine, eight per cent. Dose: H. and C., fl. 3 i.-iv.; D., m i.-v.

Unguentum Iodi, iodine ointment. Used locally only. (Composed of iodine 4, potassium iodide 1, water 2, lard 93.)

Ferri Iodidum and Syrupus Ferri Iodidi.-See under Ferrum.

Potassii Iodidum, iodide of potassium, colorless, translucent, cubical crystals, slightly deliquescent, having a peculiar, faint oujor, a pungent, saline, afterward somewhat bitter taste, and a neutral reaction; soluble in 0.8 part of water and in 18 parts of alcohol.

Sodii Iodidum, iodide of sodium, minute white or colorless, monoclinic crystals, or a crystalline powder, deliquescent on exposure to air, odorless, having a saline and slightly bitter taste and a neutral or faintly alkaline reaction. Soluble in 0.6 part water and in 1.8 parts alcohol.

Ammonii Iodidum, iodide of ammonium, a white granular salt or minute crystalline cubes, very deliquescent, and soon becoming discolored on exposure to air; odorless when white, but emitting a slight odor of iodine when colored, having a sharp 
saline taste and a neutral reaction. Soluble in 1 part of water and in 9 parts of alcohol.

These iodides should all be kept in well-stoppered glass bottles. Dose of either of them: H., 3 i.-iv.; C., 3 ij.-vi.; D., gr. iij.-x.

Unguentum Potassii Iodidi, ointment of iodide of potassium. Twelve per cent.; for local use only. Iodoformum, Iodoform (iodide of formyl). Shining, lemon-yellow crystals, having a most disagreeable persistent odor, insoluble in cold water, but soluble in 80 parts of alcohol, in 5.2 parts ether, and in chloroform, benzol, benzin, carbon disulphide, and fixed or volatile oils. Its solutions have a neutral reaction. It contains about ninety per cent. of iodine, and its odor may be disguised by balsam of Peru or oil of mirbane. Dose: H. and C., 3 ss.-i.; D., gr. iij.-viij.

Unguentum Iodoformi, ointment of jodoform, U. S. P. Ten per cent; for local use only.

Iodolum, Iodol (tetra-iodo-pyrol), a grayish-yellow powder, tasteless and odorless, containing from eighty-five to ninety per cent. of iodine. Readily soluble in ether, in hot alcohol, and in chloroform. Used as a substitute for iodoform. Dose: H., 3 ss-i. ; D., gr. i.-iij. It is a good form of iodine for internal medication, as it is practically non-irritant.

Other preparations of iodine, all for local use, are:

Iodo-Tannin, a solution of tannin in tincture of iodine.

Iodo-Phenyl, iodized phenol, or carbolized iodine, 
a mixture of carbolic acid and iodine in various proportions.

Glycerinum Tannini Iodo-Carbolizati, glycerite of iodo-carbolate of tannin, a mixture containing carbolic acid, iodine, tannin, and glycerin.

Bismuthi Subiodidum, subiodide of bismuth. See under Bismuthum.

Physiological Action.-Iodine is an irritant, stains the skin yellow, and if used in concentrated form may blister. It is also a disinfectant, deodorizer, and antiseptic, and is eliminated chiefly by the kidneys, the skin, the bronchial muccus membranes, and the salivary glands. If administered for too long: a time it produces poisoning, the animal shuns water, appears drowsy, has increased temperature, quick feeble pulse, temporary impotence, and great depression. The best and most efficient antidote is starch, which is to be followed by evacuents. The iodides all are very soluble, very -diffusible, and are rapidly excreted. Iodine and ozone are set free at the points of elimination, and thus they cause considerable irritation at these parts, especially if large doses have been given. They also occasion a profuse mucous discharge, soreness of throat and eyes, as well as irritation to the kidneys.

Both iodine and iodides induce great waste and rapid elimination of waste products, but if used for too long a time cause anæmia, emaciation, and great depression. They are classed as alteratives, resolvents, and absorbents. Iodoform, on account of the iodine it contains, is a good antiseptic and somewhat 
of a local anæsthetic. Small doses given internally are tonic and alterative. Iodol has the same action as iodoform, but is odorless, non-irritant, and practically non-poisonous to veterinary patients. It is readily soluble in the gastric juice; hence is rapidly absorbed and quickly diffused through the system.

Therapeutics of Iodine.-Catarrh and chronic nasal discharges are effectually treated with inhalations of iodine, with or withoutcarbolic acid. Inflammations of joints, rheumatic or otherwise-the tincture locally. Ringworm-the iodophenol with glycerin locally. Glandular tumors-parenchymatous injections of Lugol's solution or tincture. Bronchial filariæ- the same, with or without turpentine, intratracheally. Enlarged glands, sores, ulcers, etc., are painted with the tincture or rubbed with the ointment. Diabetes insipidus, polyuriasmall doses internally. To absorb products of inflammation in sore throat and bronchitis and to prevent adhesions; to assist in absorbing bony deposits both internally and externally, with or without mercury. Externally, as a stimulant and resolvent to bursal enlargements, in chronic synovitis, muscular pains, strains of tendons, induration of the udder. When internally administered, give one and one-half to two hours after feeding, and discontinue its use for one week when it has been regularly given for from eight to ten days.

Therapeutics OF THE Iodides.-Acute catarrh, asthma, short wind, thick wind - the potassium iodide, with Fowler's solution and belladonna. 
Chronic and capillary bronchitis-ammonium iodide, in small, frequently repeated doses. Catarrhal pneumonia-ammonium iodide and arsenic, to prevent caseation of the inflammatory products. They may be used to promote absorption of waste products, as in lymphangitis, pleuritic or other serous effusions, enlarged glands, duodenal catarrh, bronchitis, pneumonia, chronic cough, actinomycosis in cattle, purpura hemorrhagica, rheumatic affections (with colchicum), chronic lead and mercurial poisoning.

THERAPEUTICS OF IODOFORM AND IODOL.Chronic gastric and intestinal catarrh-iodol between feeding-times. Bronchitis and bronchial catarrhiodol and senega. To foul wounds, ulcers, sores, etc. -iodoform; also made into suitable suppositories and introduced into fistulæ, the rectum, and genito-urinary organs as antiseptics. To open joints, one of the best applications is equal parts of iodoform and powdered alum. Diabetes insipidus has been successfully treated with both iodoform and iodol-preferably the latter.

\section{HYDRARGYRUM-MERCURY.}

Symbol, $\mathrm{Hg}$; atomic weight, 200; quantivalence, 2 and 4 ; sometimes called "quicksilver" because of its extreme motility and metallic lustre. It is the only known liquid element, and its most common source is as a sulphide, from which it is obtained by heat to drive off the sulphur and $\mathrm{Hg}$ distils over.

Hydrargyri Chloridum Corrosivum $\left(\mathrm{HgCl}_{2}\right)$.Corrosive chloride of mercury, mercuric chloride, 
corrosive sublimate, bichloride of mercury. Heavy, colorless, rhombic crystals or crystalline masses, permanent in the air, odorless, having an acrid and persistent metallic taste, acid reaction, soluble in 16 parts of water, in 3 parts of alcohol, and in 4 parts of ether. Its solubility in water is increased by the addition of an equal weight of ammonium or sodium chloride. Four grains are neutralized by the white of one egg. It is incompatible with most substances, and therefore is best given alone. Dose: H. and C., gr. v.-viij.; D., gr. $\frac{1}{20}-\frac{1}{8}$.

ACtions AND Uses.-Alterative, caustic, corrosive, and irritant poison; antiseptic, antiphlogistic, astringent, parasiticide. Its best antidote is the white of eggs. It is used in glanders, farcy, chronic skin eruptions, œedematous swellings of the legs resulting from weed; promotes the absorption of exudates. Small repeated doses for slimy bloody dysentery in cattle. Externally to open joint as it coagulates albumin; also to indolent sores as yellow wash ( 3 ss. to aqua calcis Oi.). Concentrated solutions injected into the pipes of quittor, fistulæ; charges of from fifteen to thirty grains, with or without arsenic, to slough out tumors which cannot well be removed by the knife. Solutions of $1: 1,000$ or 2,000 as antiseptic to wounds, as dressing in foot-rot of sheep, to destroy lice, ringworm, and to allay itching of the skin.

Unguentum Hydrargyri, mercurial ointment (fifty per cent.); commonly known as blue ointment. Applied as a stimulant for indolent sores, 
ulcers, chronic and glandular swellings, mange, scab, scurvy skin diseases; for the latter usually combined with tar and sulphur. Used also to destroy lice. Must be used with care, especially if applied over a large area, as mercurial poisoning might result from absorption.

Unguentum Hydrargyri Ammoniati, ointment of ammoniated mercury or white precipitate ointment (ten per cent.), is used as a parasiticide in various forms of eczema, chronic inflammation of the joints, and conjunctivitis.

Oleatum Hydrargyri (ten to twenty per cent.); yellow oxide mercury 10 , oleic acid 90, U. S. P. Used for same purposes as the ointment, but is probably more easily absorbed; if for painful exostoses, combine with morphine.

Hydrargyrum cum Creta, gray powder, mercury with chalk (triturate thoroughly 1 part mercury with chalk 2 parts, U. S. P.). Used as antacid, laxative, and alterative. For calves or foals, in diarrhœa or bilious indigestion, five to fifteen grains on the tongue,or with gruel and ginger or other aromatic stomachics. Dose D., gr. ss. $-\mathrm{x}$.

Hydrargyri Chloridum Mite, mild chloride of mercury, mercurous chloride, subchloride of mercury, calomel, aquila alba- $\mathrm{Hg}_{2} \mathrm{Cl}_{2}-$ a white, impalpable powder, permanent in the air, odorless and tasteless, insoluble in water, ether, or alcohol. It is blackened when brought in contact with water of ammonia.

ACTIONS AND UsEs.-Irritant, stimulant, altera- 
tive, antiseptic, antiphlogistic, emetic, for dogs and pigs. In passing out of the body calomel stimulates the various excretory channels, thereby acting as a cholagogue, cathartic, diuretic, diaphoretic, and sialagogue. Applied externally as stimulant, desiccant, mild astringent, and antiseptic. It is given at the onset of acute diseases in the horse; in gastric fever with opium; in indigestion and diarrhœa of young animals; in rheumatism in horses and cattle, with quinine and salicylic acid; in jaundice, chronic liver troubles; in congested liver with aloes. Has been given in glanders and chronic skin affections. Like all mercurials it controls inflammation and promotes absorption of waste by stimulating the lymphatics. Applied externally to indolent sores and wounds; also as black wash ( 3 i. to aqua calcis Oi.). Dose: As alterative, H., Эi.-3i.; C., Đi.-3i.; D., gr. ij.-iij. As cathartic, H., 3 ss.-i., with aloes, 3 v.-vi.; C., 3 i.-ij., with Epsom salt, lb. i.; D., gr. iij.-v., with powdered jalap, gr. xx.-xl. As emetic for the dog and pig, two to three grains with same amount of tartar emetic. Has also some vermifuge effect on both the horse and dog, but is seldom given alone for that purpose.

Hydrargyri Iodidum Rubrum, red iodide of mercury, biniodide of mercury, mercuric iodide $\left(\mathrm{HgI}_{2}\right)$ a scarlet-red crystalline powder, permanent in the air, odorless and tasteless, almost insoluble in water, soluble in 130 parts of alcohol, in solution of iodide of potassium, and in solution of mercuric chloride.

ACtIon AND Uses.-Biniodide of mercury is an 
irritant similar to the mercuric chloride, but is more rapidly absorbed and more rapidly eliminated. It is used externally as an antiseptic, stimulant, counterirritant, and absorbent; generally as an ointment with lard, vaseline, or other base, from eight to sixteen per cent., with or without cantharides. The "red ointment" is used for reducing and condensing splints, ringbones, spavins, and other bony exostoses. It penetrates deeply and arrests chronic inflammation; aids in the absorption of chronic enlargements, indurated, strained tendons, bursæ, and joints. Stronger absorbent effects may be produced by combining it with iodine. Strong red ointment must be carefully used, as it causes considerable sloughing of the tissues and sometimes a permanent blemish. Again, when applied over a large surface, there may be caused acute mercurial poisoning through absorption. Symptoms of "mercurialism" are salivation, spongy gums, fetid breath, loosening of the teeth, bluish line along margins of teeth, muscular pains, fever, diarrhœa, marked anæmia, and albuminuria.

\section{Colchicum-Meadow SafFron.}

Colchici Radix, the corm or root of colchicum autumnale; Colchici Semen, the seed of colchicum autumnale. Colchicum, or meadow saffron, belongs to the natural order Melanthaceæ, and contains an alkaloid, colchicine, tannic and gallic acids, sugar, starch, and a resin. The seed gives the most reliable preparations, and is subglobular in shape, about onetwelfth of an inch thick, slightly pointed at the hi- 
lum; reddish-brown, pitted, internally whitish, very hard and tough, bitter and somewhat acrid. The root or corm is about one inch long, ovoid, flattish, and with a groove on one side, externally brown and wrinkled, internally white and solid; often in transverse slices, reniform in shape, and breaking with a short mealy fracture; odorless; taste, sweetishbitter and acrid.

Chief Preparations.-Colchici Seminis Pulvis, powdered colchicum seed; Colchici Radicis Pulvis, powdered colchicum root. Dose: H., 3 i.-ij.; D., gr. i.-v.

Extractum Colchici Seminis Fluidum, fluid extract of colchicum seed. Dose: H., fl. 3 i.-iv.; D., $3 \mathrm{ij} .-\mathrm{xv}$.

Colchicina, colchicine. Dose: H., gr. i.-v.; D., gr. $\frac{1}{120}-\frac{1}{40}$.

ACTron AND UsES.-Emetic in large doses; gastro-intestinal irritant and colicky drastic purgative. In moderate doses, diaphoretic, diuretic, and cardiac depressant while small doses decrease the amount of sweat and urine. Arterial tension is lowered and the heart is depressed by reflex action. Moderate doses increase the excretion of salts by the kidneys, especially of urea, and stimulate the flow of bile. It is used in constitutional ophthalmia, in rheumatism in the subacute or chronic form only, usually with salol or potassium iodide. In azoturea with potassium acetate or lithium citrate. In acute cerebral congestion and in ascites, full doses with opium and strophanthus. 


\section{ASTRINGENTS.}

\section{Tannic And Gallic Acids.}

Preparations.-Acidum Tannicum, tannic acid, gallotannic acid, tannin- $\mathrm{C}_{14} \mathrm{H}_{10} \mathrm{O}_{9}$. Light yellowish scales, permanent in the air, having a faint, peculiar odor, a strongly astringent taste, and acid reaction. Soluble in 6 parts of water, in 0.6 part alcohol, in 6 parts glycerin; almost insoluble in ether and chloroform. It is incompatible with alkaloids and preparations of iron. Dose: H., 3 ss.-ij.; D., gr. $\mathrm{ij} .-\mathrm{x}$.

Glycerinum Acidi Tannici, glycerine of tannin. Twenty per cent. strong; for local use only.

Unguentum Acidi Tannici, ointment of tannic acid. For local use only; made ten per cent. strong.

Acidum Gallicum, gallic acid $-\mathrm{HC}_{7} \mathrm{H}_{5} \mathrm{O}_{6} \cdot \mathrm{H}_{2} \mathrm{O}$. A nearly or quite colorless solid, crystallizing from water in long, silky needles or triclinic prisms; permanent in the air, odorless, having an astringent and slightly acidulous taste and acid reaction. Soluble in 100 parts water, in 4.5 parts alcohol, in 39 parts absolute ether, but only slightly soluble in chloroform. Dose same as tannic acid.

Differences between Tannic and Gallic Acids. -Tannin precipitates alkaloids, gelatin, albumen, gelatinized starch, and tartar emetic, while gallic acid does not, but when taken into the stomach is first converted into gallic acid before absorption. 
The tannin when oxidized being changed into gallic acid makes the difference between them seem simply that of oxidation, while if tannin is considered to be gallic acid anhydride, it would be a matter of hydration. Gallic acid may be prepared from tannin by the action of dilute sulphuric acid, but is usually obtained from nutgalls after exposure to artificial heat for a period of one month. Tannic acid is also obtained from nutgalls.

Other vegetable astringents depending on tannic or gallic acid for their therapeutical value are:

Galla, nutgall, the gall of Quercus lusitanica, caused by the punctures and deposited ova of a certain insect. They are subglobular in shape, about three-fourths of an inch in diameter, somewhat tuberculated above, otherwise smooth, heavy, and hard; sometimes showing a circular hole near the middle, communicating with the central cavity; blackish olive-green or grayish; fracture granular, grayish. In the centre a cavity containing either the partly developed insect, or pulverulent remains of it. Nearly inodorous, taste strongly astringent. Contains tannin, sixty to seventy per cent.; gallic acid, three per cent. ; besides pectin, sugar, starch, etc. Dose of the powdered galls twice that of tannic acid.

Hamamelis, witch-hazel, the leaves of Hamamelis virginica (Linné) of the natural order Hamamelaceæ, collected in autumn. Contains about eight per cent. of tannic acid, and a bitter principle; it is best known as the household preparation, Pond's extract or extract of witch-hazel. 
Quercus Albo Cortex, white-oak bark. Contains quercitannic acid, quercin, but no gallic acid.

Catechu, kutch, cutch, a dry extract from the wood of Acacia catechu, natural order Leguminosæ, a stout, climbing shrub from the islands of the Indian archipelago. Contains catechu-tannic and catechuic acids. Dose: H., 3 i.-iij.; D., gr. v.-xxx.

Tinctura Catechu Composita, U. S. P. (catechu 12, cinnamon 8 , dilute alcohol to make 100 parts of finished tincture). Dose: H., fl. $\frac{3}{\text { ss. }}-$ iij. ; D., $\Pi x x .-$ fl. $3 \mathrm{ij}$.

Kino, a gum resin obtained from the juice of Pterocarpus marsupium, natural order Leguminosæ, very similar to catechu. Contains kinotannic acid, kinoin. Dose same as of catechu.

Physiological Action. - Tannic and gallic acids, although similar in action as well as appearance and properties, differ in the degree of their action, tannin being the more powerful astringent of the two. They are both antiseptic, and their ontinued use disorders digestion, irritates mucous surfaces, and causes emaciation. Tannin enters the blood as gallic and pyrogallic acids, which change takes place in the stomach; precipitates pepsin and coagulates albumin; checks peristalsis and causes constipation. For these reasons gallic acid is preferable to tannin for internal use. Tannin, however, should be used when an antidote for alkaloidal poisoning is required, since it forms practically insoluble compounds with most of the alkaloids as well as with tartar emetic. Catechu and kino are among the best 
of the vegetable astringents, and are used to check chronic mucous discharges from the throat and alimentary canal. Hamamelis is said to have especial value in checking venous hemorrhages.

Therapeutics. - Tannic and gallic acids are useful in hematuria, albuminuria, hemorrhages from the lower bowel, catarrhal affections of mucous surfaces; also locally to ulcers, eczema, impetigo, intertrigo, and otorrhœa. Kino and catechu are very serviceable in diarrhœa and dysentery, and the former is applied locally in powder form as a styptic. Corneal ulcers are successfully treated with a weak solution of tannin; also conjunctivitis after the acute symptoms have yielded to other treatment. A decoction of oak bark is frequently used as a stimulant, antiseptic, and astringent injection to check inordinate discharges from the genito-urinary organs.

\section{Argentum-Silver.}

Chief Preparation employed in veterinary practice is:

Argenti Nitras, nitrate of silver, lapis infernalis; Argenti Nitras Fusus, lunar caustic, fused nitrate of silver. Nitrate of silver- $\mathrm{AgNO}_{3}$-is found in commerce as colorless, transparent, tabular, rhombic crystals, becoming gray or grayish-black on exposure to light in presence of organic matter; odorless, having a bitter, caustic, and strongly metallic taste and neutral reaction. Soluble in 0.8 part of water and in 26 parts of alcohol. It should be kept in dark-col- 
ored glass bottles and protected from the light. The fused nitrate of silver is prepared by melting $\mathrm{AgNO}_{3}$ at a low temperature, treating with $\mathrm{HCl}$, and pouring the melted mass into suitable moulds. Nitrate of silver is incompatible with organic matter, soluble chlorides, most mineral acids and their salts, alkalies and their carbonates, lime-water, and astringent infusions. It is always best given dissolved in distilled water. Dose: H., gr. ij.-X.; D., gr. $\frac{1}{8}-\frac{1}{2}$.

Physiological Action. - Externally, irritant, corrosive, caustic, astringent, antiseptic; internally, astringent and nerve tonic. Its continued internal administration produces gasto-in'cestinal catarrh, uræmia, albuminuria, emaciation, fatty degeneration of heart, liver, and kidneys, hemorrhages, impaired nerve functions, and death by paralysis of respiratory centres. A single large dose causes violent gastro-enteritis and ulceration of the stomach. A course of silver treatment should not exceed four or five weeks, and must be followed by purgatives and diuretics to assist in its elimination, else silver poisoning may result.

Therapeutics.-Chronic nerve troubles of dogs, such as chronic paralysis, epilepsy, chorea; chronic spinal affections causing locomotor ataxia or paraplegia. In diarrhœa and dysentery with or without opium, especially if chronic; also in chronic gastritis. In purpura in the horse it should find universal use. Locally to destroy fungoid growths, warts, to stimulate indolent wounds and ulcers, to slough out fistu$l æ$, as in quittor. In solution to foot-rot of sheep 
and cattle; in erysipelas to edges of affected part; in conjunctivitis, inflamed and suppurating eyelids (gr. ss. to fl. 3 i. of distiiled water); to remove specks and opacity of cornea if of recent origin and due to injury. (Must be carefully used on the eye, else it Lave opacities due to deposits of the metal.) Its aquevis solution as a spray in treatment of laryngeal ulcers, follicular tonsillitis, anả pharyngitis.

\section{CUPRUM-COPPER.}

Chief Preparations. - Cupri Acetas, acetate of copper, verdigris, ærugo $-\mathrm{Cu}\left(\mathrm{C}_{2} \mathrm{H}_{3} \mathrm{O}_{2}\right)_{2} \cdot \mathrm{H}_{2} \mathrm{O}$; 199.2. Deep green, prismatic crystals, yielding a bright green powder, efflorescent on exposure tc air, odorless, having a nauseating, metallic taste and acid reaction. Soluble in 15 parts of water and in 135 parts of alcohol. Dose: H., 3 i.-ij.; D., gr. $\frac{1}{4}$.2.

Cupri Sulphas, sulphate of copper, blue vitriol$\mathrm{CuSO}_{4} .5 \mathrm{H}_{2} \mathrm{O} ; 249.2$. Large, translucent, deep-blue, triclinic crystals, efflorescent, odorless, having a nauseating metallic taste and acid reaction. Soluble in 2.6 parts of water and insoluble in alcohol. Dose same as of the acetate.

Treatment of Copper Poisoning. - The best antidote to the salts of copper are the ferrocyanide of potassium and albumen. Emetics and stomach-pump if practicable; then follow with a course of potassium iodide to assist in its elimination.

Physiological Action.-Given internally, large doses produce fatal gastro-enteritis and diarrhœa; 
repeated full doses cause colicky pains and chronic intestinal irritation. Medicinal doses act as a tonic, antiseptic, astringent, and vermifuge. It is a prompt emetic for the dog. Applied externally as a stimulant, astringent, antiseptic, and caustic to foul wounds, sores, ulcers, fistulæ, farcy buds, exuberant granulations, and to check superficial hemorrhage. Given internally in farcy, glanders, purpura, chronic diarrhœa and dysentery, nasal gleet, and as a nerve tonic in dogs suffering from chorea and epilepsy.

\section{Plumbum-Lead.}

Chief Preparations.-Plumbi Acetas, acetate of lead, sugar of lead- $\mathrm{Pb}\left(\mathrm{C}_{2} \mathrm{H}_{3} \mathrm{O}_{2}\right)_{2} .3 \mathrm{H}_{2} \mathrm{O} ; 378.5$. Colorless, shining, transparent, prismatic crystals or scales, efflorescent, and attracting carbonic acid on exposure to air, having a faintly acetous odor, a sweetish, astringent, afterward metallic taste and faintly acid reaction. Soluble in 1.8 parts of water and in 8 parts of alcohol. Dose: H., 3 ss.-i.; D., gr. i.-iij.

Liquor Plumbi Subacetatis, acetum plumbi, solution of subacetate of lead, Goulard's extract. Prepared by boiling together for a half-hour lead acetate $1 \%$, lead oxide 12 , and distilled water to make 100 parts; to be filtered when cool. This forms a perfectly clear alkaline liquid, which, however, sometimes becomes turbid on standing.

Liquor Plumbi Subacetatis Dilutus, aqua plumbi, diluted solution of subacetate of lead, lead water (Goulard's extract 3, water $9{ }^{\prime y}$ ). 
Liquor Plumbi et Opii, solution of lead and opium. A local sedative, anodyne, and astringent application (Goulard's extract $\xi$ ss., laudanum $\xi$ iss., water इ xiv.).

Chronic lead-poisoning produces a bluish line along the margins of the gums, an unsteady gait, swellings around the articulations (with heat or tenderness), impaired sensibility, difficult breathing, in some animals constipation, in others diarrhœa with colicky pains. It is best treated with sodium iodide to saturation. The antidotes for acute lead-poisoning are Epsom salt or Glauber salt; the stomach-pump if practicable; albuminous drenches and henbane or opium to relieve irritability.

Physiological Action.-Lead salts are astringent and styptic if given internally; externally, astringent, mildly antiseptic, anodyne, and slightly stimulate to absorption of deposits of inflammatory products.

Therapeutics. - In eczema, erythema and impetigo, combined with glycerin, carbolic acid, and water. To burns, the carron oil with Goulard's extract or Goulard's extract and linseed oil. To sprains and strains, also painful swellings, the liquor plumbi et opii ; catarrhal discharges of muco-purulent character; to wounds and abrasions. In internal hemorrhages, the acetate; the same with or without opium in diarrhœa and dysentery. Should not be applied to the eye, as it may leave a deposit which is difficult to remove. 


\section{ZINCUM-Zinc.}

Chief Preparations.-Zinci Acetas, acetate of zinc. Soft, white, micaceous or pearly, six-sided tablets or scales, somewhat efflorescent in dry air, having a faintly acetous odor, a sharp, metallic taste, and a slightly acid reaction. Soluble in 3 parts of water and in 30 parts of alcohol. Is employed mostly externally. Dose, same as of the sulphate.

Zinci Carbonas, carbonate of zinc. A white, impalpable powder, permanent in air, odorless, tasteless, insoluble in water or alcohol. Dose: H., 3 ij.-iv.; D., gr. ij.-vi.

Zinci Chloridum, chloride of zinc. A white, crystalline powder, or white, opaque pieces, very deliquescent, having a very caustic, saline, and metallic taste and acid reaction. Soluble in both alcohol and water. It should be kept in small glass-stoppered bottles. It is used externally only.

Zinci Oxidum, zinc oxide. A soft, pale-yellowish, or nearly white powder, permanent in air, odorless, tasteless, insoluble in water or alcohol. Dose same as of the carbonate.

Zinci Sulphas, sulphate of zinc, white vitriol. Small, colorless, right rhombic prisms or acicular needles, slowly efflorescing in dry air, having a sharp, saline, nauseous, and metallic taste and acid reaction; soluble in 0.6 part of water, insoluble in alcohol. Dose: H., 3 ss.-ij.; D., gr. i.-iij. (as an emetic in water, gr. viij.-xv.). 
Physiological Actions. - The zinc salts are mild astringents, being not so powerful as the corresponding salts of lead, and do not exhibit the tendency to accumulate in the system which is peculiar to most metallic salts. The acetate, chloride, and sulphate are known as the "soluble zinc salts," and if given in overdoses will cause violent gastro-enteritis. The acetate given internally is a nerve tonic and emetic; externally, stimulant and astringent. It is formed in the well-known "white wash" (plumbi acetici, zinci sulphurici, āā $\bar{z}$ i.; aquæ, Oi.). The chloride, internally, is a powerful irritant poison; externally, antiseptic, astringent, stimulant, and caustic. Employed also as disinfectant and deodorizer, and is known by many as "zinc butter." The sulphate, internaily, as a tonic, but is inferior to iron. As an internal astringent it is more powerful than the lead or silver salts; also used as an emetic. Externally, stimulant, astringent, and antiseptic.

The oxide and carbonate are mild astringents, protectives, and desiccants. The long-continued use of the salts of zinc may at times produce symptoms resembling those of lead-poisoning, but not so severe, and yielding more rapidly to the usual treatment.

Therapeutics. - To inflamed and catarrhal conditions of mucous membranes, weak solutions of the acetate or sulphate. In conjunctivitis, a collyrium of the acetate or sulphate, with or without atropine (grs. i.-iv. of the zinc salts to the ounce of distilled water). To relieve the tenderness and itching of skin diseases, the oxide or carbonate made into an oint- 
ment with tar and vaseline; diarrhœea and dysentery, the sulphate together with opium; chorea, if recent, in the dog, the sulphate; if chronic, the iron salts are preferable. A solution of the sulphate with a small amount of sulphuric acid may be used to check local hemorrhage, excessive perspiration, or mucous discharge. To wounds, sores, ulcers-the white wash or the sulphate in solution. The chloride as a caustic to poisoned wounds, unhealthy granulations, footrot in sheep; as a wound dressing, two to three per cent. Epilepsy-the oxide in small doses. As a prompt and efficient emetic in narcotic poisoning, the sulphate dissolved in warm water.

\section{ALUMEN-ALUM.}

The alums are a series of compounds of aluminum with a sulphate of an alkaline metal or group (potassium, sodium, or ammonium). The official preparation is the potassium-alum or potassic-aluminic sulphate, and is a combination of the sulphate of aluminum with the sulphate of potassium; it is therefore called a double sulphate.

Preparations.-Alumen, alum, potassium alum. Occurs as large, colorless, octahedral crystals, sometimes modified by cubes, acquiring a whitish coating on exposure to air, odorless, having a sweetish, astringent taste, and an acid reaction. Soluble in 10.5 parts of. water, insoluble in alcohol. When gradually heated it gives off twenty-four molecules of water, and becomes dried alum. Dose: H., 3 ij.-iv.; D., gr. $\mathbf{x} .-\mathrm{xx}$. 
Alumen Exsiccatum, Alumen Ustum, dried alum. A white granular powder, attracting moisture when exposed to the air, odorless, having a sweetish, astringent taste, and slowly but entirely soluble in 20 parts of water.

Incompatibles are salts of iron, manganese, bismuth, antimony, lead, and salts of most of the alkaloids; it is therefore best given alone.

Physiological Actions.-Alum is a very mild caustic, a good astringent, but is not so active as the copper and zinc salts. It stimulates muscular contraction, coagulates albumin, at first excites the flow of saliva, and then greatly diminishes it. Having the power to coagulate and precipitate pepsin, it arrests digestion, stops peristalsis, and usually causes constipation. Large repeated doses act as a violent gastro-intestinal irritant and frequently cause diarrhœa. Weak solutions coagulate albumin, but enter the blood, constrict the capillaries, check secretions, especially those of mucous surfaces, and arrest capillary hemorrhages. Alum is also given as an emetic, is very efficient, and leaves no depressant after-effects.

Therapeutics. - Small doses are serviceable in gastric catarrh and to check hemorrhage at distant points. Leucorrhœa and gonorrhœa are satisfactorily treated with injections of alum, zinc sulphate, and borax. To ulcers, sores-the dried alum as a stimulant and caustic. Aphthous, ulcerated conditions of the mouth, sore throat-spray, gargle, or wash of alum with carbolic acid. Catarrhal ophthalmia, in the acute stage-a collyrium of alum grs. v. to the 
ounce of distilled water. Open joint, one of the most satisfactory treatments-equal parts of dried alum and iodoform. Uterine hemorrhages, post-partum hemorrhage-douche of alum eight ounces to one gallon water, and ergot and tincture of opium internally. Diarrhœa and dysentery-alum with tincture of opium; but lead salts and vegetable astringents are more effectual. Alum has been given with some success in hæmaturia and polyuria. As a very good emetic for the dog, $3 \mathrm{i}$. in warm water. Pipe-clay or argyl, and fuller's earth, both preparations of aluminum silicate, are mild astringents, and are used as dusting powders for simple wounds, harness galls, etc.

\section{MOTOR EXCITANTS.}

\section{Nux Vomica.}

Source ANd Composition.--The seeds of strychnos nux vomica, an East Indian tree, belonging to the natural order Loganiaceæ. It contains two alkaloids, strychnine and brucine, the former being the more important, which are combined with igasuric acid in the plant. They are also found in lesser quantity in other plants of this order. Brucine, although corresponding physiologically as well as therapeutically with strychnine, is only one-twelfth as strong, while strychnine is fully fifty times more active than the powdered nux vomica. Nux vomica is sometimes known as Quaker button.

Chief PreParations.-Extractum Nucis Vom- 
icce, solid extract of nux vomica. Dose: H., gr. iv.-viij.; C., gr. viij.-xvi.; D., gr. $\frac{1}{8}-\frac{1}{2}$.

Extractum Nucis Vomica Fluidum, fluid extract of nux vomica. Dose: H., 3 ss.-i. ; C., fl. 3 ss.-ii. ; D., $\Pi$ ss. $-\mathrm{iij}$.

Tinctura Nucis Vomica, tincture of nux vomica, twenty per cent. Dose: H., fl. 3 ijss.-v.; C., fl. 3 iij. -f. 亏亏i.; D., Tl ij.-x.

Pulvis Nucis Vomicoe, powdered nux vomica. Dose: H., 3 ss.-i. ; C., 3 i.-ij.; D., gr. ss.-ij.

Strychnince Sulphas, strychnine sulphate. Dose: H., gr. i.-ij.; C., gr. ij.-v.; D., gr. $\frac{1}{60}-\frac{1}{20}$.

If used subcutaneously, one-fourth to one-half of these doses.

Sulphate of strychnine occurs as colorless or whitish, shining, prismatic crystals, efflorescent in dry air, odorless, having an intensely bitter taste and neutral reaction. Soluble in 10 parts of water, in 60 parts of alcohol, and in 26 parts of glycerin, but insoluble in ether.

Antidotes for Strychnine Poisoning.-Tannic acid to form the insoluble tannate; emetics or stomach-pump, if possible; absolute rest. Evacuate bladder frequently to prevent reabsorption. Antagonists are chloral, chloroform, and physostigmine.

Physiological Actions. - Small doses act as a bitter tonic, stimulate respiration, secretion, appetite, and digestion; increase peristalsis, stimulate the heart, respiratory and vasomotor centres, so that arterial tension is raised and the arterioles are contracted. The rapidity and depth of the respirations 
are increased, as is also the body temperature to a slight degree. Full doses dilate the pupils, relax the arterioles, lower blood pressure, produce spasmodic respirations, twitching of the muscles, and jerking of the extremities. Toxic doses, especially if given on an empty stomach, rapidly produce tonic spasms, particularly of the extensor muscles, rapidly succeeding each other on the least irritation, with intervals of repose (differential diagnosis from tetanus, where the spasm is constant).

Death takes place very rapidly in one or two hours, according to the size of the dose. Very large doses kill almost instantly, death taking place by $\mathrm{CO}_{2}$ poisoning, as the respiratory muscles become completely paralyzed.

Strychnine exalts all the functions of the cord. If given in larger doses there is over-stimulation; in further increased doses the entire cord is suddenly paralyzed-i.e., the spinal functions are destroyed at one blow. Strychnine is absorbed more rapidly from the rectum than from the stomach, and still more rapidly from the bronchi and cellular tissues. It is slowly excreted in an unchanged condition in the urine.

TheraPeutics.-Nux vomica and strychnine are among the very best of the respiratory, cardiac, muscular, and nerve stimulants and tonics. Strychnine is a powerful antagonist in poisoning by opium or morphine. In anæmia, chlorosis, and convales cence from debilitating diseases, strychnine combined with iron and quinine; tetanus; atonic 
dyspepsia; gastric catarrh; chronic or habitual constipation, and colic resulting therefrom; to assist in the action of purgatives. For local paralysis, inject strychnine into affected muscles once or twice a week. Post-partum paralysis and chronic paralysis of cattle; dyspnœea from pulmonic affections; also in roaring and broken wind. Cardiac failure from any cause; cerebro-spinal meningitis; stringhalt; chorea, asthma, and chronic bronchitis in dogs. Strychnine stimulates the sexual functions if continued for some weeks. Combined with cantharis in small doses it is an effectual remedy to relieve relaxation of sphincter muscles of the bladder.

\section{Ergota-Ergot.}

Ergota, ergot of rye, Secale cornutum, horned or spurred rye. The sclerotium of Claviceps purpurea, natural order Fungi, replacing the grain of Secale cereale, natural order Graminaceæ. Somewhat fusiform, obtusely triangular, usually curved, about one inch long and one-eighth inch thick, three-furrowed, obtuse at both ends, purplish-black in color, whitish internally with some purplish striæ, breaking with a short fracture; having a peculiar, heavy odor and a disagreeable, oily taste. It contains three distinct active principles: 1 . Ergotic or ergotinic acid, having no power to contract muscular fibre, but acting on the heart, respiration, nerves, and producing convulsions. 2. Sphacelinic or sphacelic acid, soluble in alcohol, which stimulates vasomotor centres, and is 
the chief agent in producing gangrene of the extremities in chronic ergotism. 3. Cornutine, an alkaloid, which produces convulsions, ergotism, and increased peristalsis of the uterus. Ergot also contains a nondrying oil, with trimethylamine, and lactie and phosphoric acids.

Preparations of Ergot.-Ergota Pulvis, powdered ergot. Dose: H., 3 ss. - i. ; D., gr. xv. $-\mathrm{xxx}$.

Extractum Ergotce, solid extract of ergot, ergotin. Dose: H., gr. xx.-xl.; D., gr. i.-v. (prepared by evaporating fluid extract 5 parts to 1 part).

Extractum Ergotoe Fluidum, fluid extract of ergot. Dose: H., fl. 3 iij.-viij.; D., Tl x.-xx.

Ergotole, a very concentrated fluid extract, is manufactured by Messrs. Sharp \& Dohme, of Baltimore, Md. It is two and one-half times stronger than the official fluid extract, and contains in permanent solution all the active principles of ergot in an unchanged condition and permanent form, while the inert and irritating constituents have been removed. It does not produce nausea even in repeated large doses, and being non-irritant is especially valuable for hypodermic medication. Dose: H., 3 ss.-iij.; D., $\pi$ v.xxx.

Medicinal ACtions and Uses.-As a parturient, but should not be used until after the head and neck of foetus have passed the os uteri. Ergot acts as a motor excitant, hæmostatic, stimulating chiefly the spinal cord, and effecting a contraction of the muscular coat of vessels, intestines, and particularly of the uterus; in fact, its action is directed mostly to wher. 
ever we find unstriped muscular tissue. Large doses produce cerebral and spinal anæmia. The main factor in producing the gangrene of ergotism is the contraction of the arterioles. Acute ergotism in the horse manifests itself by salivation and colicky pains; chronic ergotism causes convulsions and gangrene of the extremities. The extremities at first become numb and cold; soon a small bleb or blister is formed an inch or two above the coronet. This blister has a bluish tint, breaks, suppurates, and gangrene is established.

Inflammations of mucous surfaces are well treated with ergot both internally as well as locally; acute dysentery in the congestive stage; arterial hemorrhage-ergot is one of the best remedies. In parturient apoplexy, cerebro-spinal meningitis, phrenitis and encephalitis, aneurism, cardiac hypertrophy without valvular lesion, myelitis, spinal congestion, incontinence of urine, and chronic metritis. Injections of ergotole to reduce aneurismal sacs, fibroid tumors, etc.

\section{Digitalis-Foxglove.}

The leaves of Digitalis purpurea, natural order Scrophularineæ, collected from plants of the second year's growth. They are from four to twelve inches long, of a dull-green color; odor faint and tea-like, taste bitter and nauseating. The plant grows wild in Europe, is cultivated in this country in gardens for its beautiful spike of purple flowers, and largely by the Shakers for the drug market. According to the most accurate analysis available (Schmiedeberg's), digi- 
talis contains five principles-viz. 1. Digitalin, amorphous, soluble in alcohol, insoluble in water, and is the active ingredient of digitalinum, U. S. P. 2. Digitoxin, insoluble in water, slightly soluble in alcohol, but the most active of all. 3. Digitalein, soluble both in water and alcohol. 4th. Digitonin, soluble in water, but only slightly soluble in alcohol. It is quite active, but, like saponin, forms a solution which froths readily and antagonizes the three first mentioned. 5. Digitin, which is apparently inert. The first three are cardiac poisons, the fourth antagonizes them; all five are non-nitrogenous, and, except digitin, are glucosides. Digitalis contains no true alkaloid.

Preparations.-Folia Digitalis Pulvis, powdered digitalis leaf. Dose: H., gr. xv. -3 ss.; D., gr. i.-iij.

Extractum Digitalis, solid extract of digitalis. Dose: H., gr. v.-xv.; D., gr. $\frac{1}{6}-\mathrm{i}$.

Extractum Digitalis Fluidum, fluid extract of

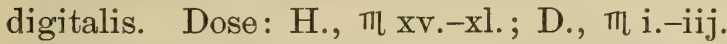

Tinctura Digitalis, tincture of digitalis (fifteen per cent.). Dose: H., fl. 3 ij.-v.; D., Tl iij.-xiij.

Infusum Digitalis, infusion of digitalis. Dose: H., fl. 3 ij.-iv.; fl 3 i.-iv. (digitalis leaf 3, cinnamon 3 , boiling water 185 , alcohol 15 , water to make 200 parts).

Digitalinum, digitalin. Dose: H., gr. $\frac{1}{4}-\frac{1}{2}$; D., gr. $\frac{1}{60} \frac{1}{30}$.

Physiological Action.-Digitalis is a cardiac tonic and vascular stimulant, a motor excitant, para- 
lyzant, anaphrodisiac, diuretic, and emetic. Overdoses irritate the mucous membranes, causing sneezing, severe gastric disturbance, nausea, vomiting in some animals, colicky pains and purgation, the discharges being of a greenish color. It reduces temperature, presumably by decreasing the blood supply to the tissues. Digitalis slows the heart, but increases the force of the heart muscle. It stimulates the cardiac motor ganglia, the inhibitory apparatus, and vasomotor centre, contracting the arterioles, and thereby greatly raising arterial tension. Continued full doses exhaust the irritability of the motor ganglia and paralyze the cardiac muscle itself. It decreases the sexual appetite, lessens the contractile power of striped muscular tissue, causes weakness and languor; the excretion of urea, while at first increased, is very soon much diminished. The diuretic action of digitalis is due to its peculiar influence upon the general and renal circulation. It greatly increases blood pressure in the kidneys, as well as the rapidity of the renal circulation. Toxic doses decrease the reflexes, paralyze the muscles and the peripheral nerves, motor and sensory; respirations become rapid and feeble; cyanosis, coma, and convulsions follow; death takes place by sudden paralysis of the heart, which is arrested in systole (aconite arrests heart in diastole).

The curative effects of digitalis are brought about in some of the following ways: 1st, By strengthening the heart's action; $2 d$, by reducing the strength of the heart beats if that organ is acting too powerfully; 
$3 \mathrm{~d}$, by lessening the frequency of the heart beats; 4th, by correcting irregularity in the heart's action.

Antidotes and Antagonists.-The best chemical antidote is tannin, but the stomach-pump or emetics must be used if possible, as the tannate of digitalin is not entirely inactive. The best antagonist for large doses is aconite, while opium is very serviceable to counteract the effects of its long-continued use.

Incompatibles are tannic acid, most of the iron preparations, and cinchona.

TheraPeUtics.-Enfeebled or irregular heart's action during influenza; mitral disease; in hypertrophy of old horses when overworked, with weak pulse; difficult breathing and dropsical effusions if resulting from weak heart; palpitation from overwork or exertion after a full meal; pericarditis; endocarditis; hemorrhage from lungs, stomach, or from large surfaces. In pneumonia in earlier stage, to help relieve the local engorgement. In treating thick wind or broken wind; coughs; combined with potassium nitrate or acetate as a diuretic; various dropsical affections. The chief indications for the use of digitalis are enfeebled, irregular, or irritable heart, deficient arterial pressure, venous engorgement, .and scanty secretion of urine. Digitalis should not be used in aortic disease or hypertrophy if pulse is strong, firm, and regular. It has been recommended in purpura, combined with copper sulphate in snall doses. According to some authors digitalis is cumulative in action, but personal experience fails to bear this out. 


\section{Strophanthus.}

The seeds of Strophanthus hispidus (natural order Apocynaceæ), an African climbing plant, from which the natives prepare a deadly arrow poison known as kombé. It contains from eight to ten per cent. of strophanthin, a crystalline glucoside, which is its active principle.

Preparations.-Tinctura Strophanti (five per cent.), tincture of strophanthus. Dose: H., fl. 3 i.ij.; D., m i.-v.

Strophanthinum, strophanthin. Dose: H., gr. $\frac{1}{10}-\frac{1}{2} ;$ D., gr. $\frac{1}{120}-\frac{1}{60}$.

Physiological Action.-Strophanthus acts very energetically upon muscular tissues, increasing the contractile power of all striped muscle. Its action is brought about by direct contact through the blood, and consequently, as the heart receives more blood than any other part of the organism, it is rapidly and strongly affected by doses too small to show much effect elsewhere; for this reason small doses stimulate the heart's contractions and lower the rate of the heart beats; at the same time blood pressure is raised and diuresis is produced. Large doses paralyze the heart, and if this has once taken place there is no way of relief, as the heart becomes so contracted as to seem almost of the rigidity of stone. Strophanthus is the most powerful and most rapid heart stimulant known, being much more powerful than digitalis, from which it differs in that it does not cause any 
vasomotor constriction of the arterioles. It reduces the pulse, helps to lower body temperature, does not create any gastro-intestinal disturbance, and is not cumulative in action. It rapidly relieves cardiac dyspnœa, and its influence upon the circulation will last for a long time. According to Professor Frazer its effects have been known to continue for as long as eight days. It may be beneficially given in palpitation or exaggerated cardiac action, as from overwork or weak heart, to relieve dyspnœa of asthma, pulmonary œdema, acute endocarditis, chronic Bright's disease, and valvular lesions of the heart.

\section{Convallaria-Lily of the Valley.}

The well-known perennial plant, "lily of the valley," Convallaria majalis, belonging to the natural order Liliaceæ. All parts of the plant are used, but preparations of the root seem most active. It contains two active glucosides-viz.: Convallamarin, the cardiac-acting principle, soluble both in alcohol and in water; and convallarin, an emeto-cathartic principle, soluble in alcohol but insoluble in water; also an acrid resin resembling convallarin in action. The more important preparations of convallaria are:

Extractum Convallarice Fluidum, fluid extract of convallaria. Dose: H., fl. 3 ss.-ij.; D., m ij.-x.

Convallamarinum, convallamarinum. An amorphous, white, bitter powder, soluble in water and in alcohol, but insoluble in ether. Dose: H., gr. x.xxx.; D., gr. ss.-ij. 
Physiological Action.-Convallaria is a very valuable heart tonic, diminishing the frequency and increasing the strength of the heart beat, raises arterial tension, slows and deepens the respirations, and increases the amount of urine excreted. Unlike digitalis, it has no cumulative action, but very closely resembles that drug in its action as a cardiac tonic and as a diuretic. In over-doses it is an active poison, causing irregular cardiac action, gastro-enteritis, rapid feeble pulse, and greatly lowered blood pressure, slowed and labored respirations, and finally heart arrest in systole, by direct stimulation of its inhibitory apparatus.

Therapeutics.-Convallaria is considered safer than digitalis, and may be employed in all cases where the latter drug is indicated. In cardiac neurosis it frequently gives prompt relief when digitalis has failed.

\section{Belladonna-Deadly Nightshade.}

The leaves and root of Atropa belladonna, a European plant, natural order Solanaceæ, and containing two alkaloids. The more important of these, atropine, the active principle, is in general use; while the other, known as belladonnine, is but seldom used for its therapeutic properties. The plant contains also malic acid and a coloring matter known as atrosin.

Derivatives of Atropine.-Atropine may be resolved into tropin and tropic acid, both of which, as well as atropine, may be produced synthetically. Homatropine is the product resulting from treating 
the amygdalate of tropin with dilute $\mathrm{HCl}$. Its hydrobromate is much used by ophthalmologists, and is a cardiac depressant.

Chief Preparations.-Pulvis Belladonnoe Foliorum, powdered belladonna leaf. Dose: H., 3 iv.亏 i.; D., gr. v.-x.

Tinctura Belladonnce Foliorum, tincture of belladonna leaf (fifteen per cent.). Dose: H., fl. 3 ij.f. 3 i.; D., Mij.-xxx.

Extractum Belladonno Foliorum Alcoholicum, alcoholic extract of belladonna leaves. Dose: H., gr. v. -xv. ; D., gr. $\frac{1}{10-\frac{1}{2}}$.

Extractum Belladonno Radicis Fluidum, fluid

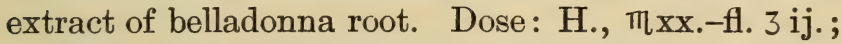
D., Mi. $-\nabla$.

Unguentum Belladonnce, ointment of belladonna. For local use only. (Alcoholic extract of belladonna 10, diluted alcohol 6 , benzoinated lard 84.)

Linimentum Belladonnoe, liniment of belladonna. For local use only. (Fluid extract of belladonna root 95, camphor 5.)

Atropinoe Sulphas, sulphate of atropine. Dose: H., gr. ss.-iss.; D., gr. $\frac{1}{120-\frac{1}{60}}$. Hypodermically, one-fourth to one-half of these doses.

Sulphate of atropine is a white, indistinctly crystalline powder, permanent in the air, odorless, having a very bitter, nauseating taste and a neutral reaction. Soluble in 0.4 part of water and in 6.5 parts of alcohol.

Physiological Actions.-Belladonna is a powerful mydriatic, an irritant narcotic, an antispas- 
modic, and anodyne. It is a stimulant to the heart, respiration, and spinal cord if given in small doses, while large doses paralyze the secretory and motor nerve endings, but stimulate the sympathetic system. Its internal administration produces a dryness of the mucous membranes of the nose, mouth, throat, and larynx; while the gastric and intestinal secretions, although at first decreased, are soon reproduced in increased quantity. The heart rate at first is slowed, but shortly becomes very rapid and vigorous, the pulse being doubled in rapidity, arterial tension is raised, and circulation increased. This is brought about by belladonna directly stimulating the cardiac sympathetic and paralyzing the intracardiac inhibitory ganglia, which, while stimulating the accelerator apparatus, decreases the inhibitory power. Like other members of the same group, belladonna stimulates the vasomotor ganglia over the entire body, with paralysis by over-stimulation; thus the heart weakens, vessels become relaxed, blood pressure is greatly lowered, and if the action of the drug is allowed to continue, complete motor paralysis ensues, with delirium, stupor, and finally death by asphyxia (paralysis of respiration). Belladonna lessens intraocular pressure, and by its systemic as well as local administration dilates the pupil of the eye. The dilatation reaches its maximum in the dog in from twenty to twenty-five minutes, in herbivora in from one-half to three-fourths of an hour, and its effects may continue in a lesser degree for several days. The brain is congested by belladonna and more or 
less delirium is produced, with hallucinations, mental disorder, and a tendency to irregular movements caused by a selective action of the drug upon the cells of the gray matter. The spinal cord is stimulated from the second cervical to almost the last dorsal vertebra, resulting finally in paralysis of both central and peripheral motor nerves, locomotory power being first lost in the posterior extremities. Sensation is somewhat impaired, but muscular irritability is unaffected. By the increased circulation metamorphosis is greatly promoted, which, together with the increased respiration, raises the temperature. A scarlatina-like rash (seldom discernible in the lower animals) with dysphagia and sore throat, is occasionally produced by belladonna on the skin and fauces, and is due to capillary congestion resulting from the increased circulation. Belladonna is rapidly diffused and rapidly eliminated, which process is accomplished chiefly by the kidneys. Large doses diminish the excretion of urine, while small doses seem to be without effect in that particular. Since atropine is eliminated in an almost unchanged form, the urine of an animal under its action will dilate the pupil of another animal. The herbivora are not so profoundly affected by belladonna as the carnivora, the goat being accredited with the ability of consuming quantities of the leaf with impunity. On the dog the cardiac action of the drug is more apparent, while in the horse the cerebral action predominates.

Antidotes and Antagonists.-Belladonna has been thought to have no direct physiological antago- 
nist, but opium counteracts its effects on the cerebrum, heart, respiration, arterial tension, pupil, and kidneys; while aconite, eserine, pilocarpine, and quinine, each antagonize some of its effects. Later investigations, however, have shown that muscarine, an active toxic alkaloid from Agaricus muscarius, the fly-agaric, is a complete, prompt, and perfect physiological antagonist to atropine. In treating belladonna poisoning, tannic acid, emetics, and the stomachpump when practicable, should be employed.

Therapeutics.-Belladonna is used to relieve the pain attendant upon various inflammations, rheumatism, cancer, pelvic affections; to stimulate the weakened heart, as in influenza; in catarrh, pharyngitis, laryngitis, and bronchitis. Very serviceable in the second stage of respiratory diseases in the horse, when secretion is abundant, deglutition difficult, and the throat sore and irritable. Spasmodic cough of bronchitis and distemper is very amenable to belladonna with ammonium carbonate. Spasmodic colic is frequently treated with belladonna and camphor and chloral, or belladonna with aconite and solution of acetate of ammonia. In constipation-combined with nux vomica and aloes; also given to assist in the action of other purgatives and prevent their griping. In tetanus-belladonna with alcohol in large doses; also the extract smeared upon the teeth. In cerebral and spinal hyperæmia, encephalitis, meningitis, and myelitis-with ergot and use of cerebral or spinal ice bag. Given internally or as an injection, belladonna relieves irritation of the bladder and urin- 
ary apparatus, rectum, and uterus. Rigidity of os uteri in retarded or delayed parturition has been successfully treated by direct local application of extract of belladonna. In mammitis, to check the milk secretion, allay inflammation and pain-the fluid extract and camphor liniment, or liniment of belladonna. In treating abscesses, boils, carbuncles, and other superficial inflammations, apply locally; the same when it is desired to determine and increase the action of belladonna given internally-i.e., in tetanus apply belladonna liniment along the spine; in spasmodic cough, the same to the throat; in diseases of the kidneys, over the loins, etc. Glandular swellings may be treated locally with mercurial ointment and extract of belladonna. In diseases of the skin to relieve itching; in erysipelas, locally as well as small frequently repeated doses internally. Epilepsy and convulsions of canine patients may be treated with belladonna and sodium bromide. In applying belladonna over very large surfaces, especially upon the dog, great care must be exercised lest the animal become poisoned by absorption. The attendant when rubbing a patient with belladonna liniment or other powerful preparation of belladonna must be cautioned to thoroughly cleanse his hands after using the preparation, lest he too suffer from the absorption.

\section{Hyoscyamus-Henbane.}

The leaves and tops of Hyoscyamus niger, a biennial plant, natural order Solanaceæ, growing wild in many parts of Great Britain. It is also cultivated 
for the drug market. Its active principle is an alkaloid called hyoscyamine, which is probably identical with atropine. From it is derived hyoscine, a semiliquid alkaloid yielding tropic acid and pseudotropine.

Preparations. - Extractum Hyoscyami, solid extract of henbane. Dose: H., 3 ss.-i.; D., gr. ss.-ij.

Extractum Hyoscyami Fluidum, fluid extract of henbane. Dose: H., fl. 3 iss.-vi.; D., $\pi$ v.-xx.

Tinctura Hyoscyami, tincture of henbane. Dose: H., fl. 亏 i.-iij.; D., fl. 3 ss.-iij.

Hyoscyamince Sulphas, hyoscyamine sulphate. Dose: H., gr. ss.-v.; D., gr. $\frac{1}{40}-\frac{1}{2}$. Hypodermically, one-half of these doses.

Hyoscince Hydrobromas. Dose: H., gr. $\frac{1}{8}-\frac{1}{4}$; D., gr. $\frac{1}{100}-\frac{1}{50}$.

Incompatibles are liquor potassæ and all other fixed caustic alkalies.

Actions and Uses fo Hyoscyamus. - It is similar in action to belladonna, but not so powerful. Is a good calmative and hypnotic, anodyne and antispasmodic, and is sometimes used like atropine as a mydriatic. Full doses stimulate the brain centres and paralyze motor nerve endings; over-doses destroy life by paralyzing the respiratory function. Hyoscyamus does not check peristalsis, and therefore is useful as a hypnotic and anodyne in "irritable conditions of the alimentary canal and urinary apparatus when opium is contraindicated. It is frequently combined with cathartics to enhance their activity and at the same time to prevent griping. It is prescribed as an 
anodyne in colic, to allay irritable cough, in epilepsy and chorea, in locomotor ataxia, and since it is mainly excreted by the kidneys its value to relieve irritability of these organs and of the bladder will be apparent.

\section{CEREBRAL EXCITANTS.}

\section{CAMPHORA-CAMPHOR.}

Camphor is a stearopten (solid volatile oil), obtained from Cinnamomum camphora, natural order Laurineæ, a native tree of Japan, China, Borneo, etc., and is purified by sublimation. Found in the market as white translucent masses of a tough consistence and crystalline structure, readily pulverizable in the presence of a little alcohol, ether, or chloroform. Its specific gravity is 0.99 , it sublimes without residue, burns with a smoky but luminous flame, floats on water, and if exposed to air slowly evaporates. It has a penetrating odor and a pungent taste, is soluble in 1,300 parts of water, readily soluble in alcohol, ether, chloroform, fixed and volatile oils, milk, and acetic acid.

Preparations. - Camphora Pulvis, powdered camphor. Dose: H., 3 i.-iij.; D., gr. v.-x.

Aqua Camphorce, camphor water, used chiefly as a sedative collyrium. (Camphor 8, alcohol 16, water 976.)

Spiritus Camphorce, spirit of camphor, principally for local use. (Camphor 10, water 20, alcohol to make 100.) 
Linimentum Camphorce, Oleum Camphoratum, liniment of camphor or camphorated oil. For local use. (Camphor 1, cotton-seed oil 4.)

Linimentum Saponis, Linimentum Camphorce Compositum, Tinctura Camphorce Composita, Spiritus Saponis Camphoratus, soap liniment, compound camphor liniment, compound tincture of camphor, camphorated soap spirit, opodeldoc. For local use only. (Soap in shavings 10, camphor 5, oil of rosemary 1 , alcohol $\%$, water to make 100.)

Camphora Monobromata, monobromated camphor. Used chiefly in canine practice. Dose for dog, gr. i.-x.

Physiological Action.-Camphor is antispasmodic, antiseptic, anodyne, a stimulant expectorant, diaphoretic, a cerebral excitant, and gastro-intestinal irritant. Externally, counter-irritant, stimulant, antiseptic, parasiticide. It has an acrid, hot taste, irritates the skin and mucous membranes, and if given in large quantity causes severe gastric disturbance with all the effects of an irritant poison. Moderate doses stimulate both the vasomotor system and the cardiac motor ganglia, at the same time decreasing the influence of the pneumogastric, in this way increasing circulation and raising arterial tension; mental activity, respiration, and the sweat glands are stimulated, pain allayed, the menstrual flow and sexual appetite increased; while large or continued doses depress the generative function and occasionally cause dysuria. Its anaphrodisiac effects are not very pronounced in the lower animals. Large doses 
depress the entire central nervous system, depress the heart, lower arterial tension, diminish the reflex functions of the cord, produce coldness of the surface, insensibility, coma, convulsions, and sometimes death. Camphor is eliminated chiefly by the bronchial mucous membrane and skin; also by the kidneys.

Therapeutics. - In diarrhœa-with ether, laudanum, and aromatics. In enteritis-with opium. To counteract cardiac depression and as a febrifugewith sweet spirit of nitre and solution of acetate of ammonia. To relieve vomiting; in nymphomania, typhoid fevers; in bronchitis, spasmodic cough, sore throat, and other affections of the respiratory apparatus. In gangrene internally and locally. The monobromated camphor in chorea of the dog. Externally, as a stimulant, counter-irritant, and to allay itching in skin diseases, as in eczema and urticaria.

\section{ASAFGTIDA-ASAFGeTIDA.}

A gum-resin obtained by incision from the living roots of Ferula narthex and of Ferula scorodosma (natural order Umbelliferæ, Orthospermæ), an Afghan plant. Its principal constituent is a sulphuretted volatile oil, consisting chiefly of allyl sulphide. It also contains a gum and a resin, with feruliac, malic, acetic, formic, and valerianic acids.

Asafcetida occurs in irregular masses, composed of whitish tears, which are embedded in a yellowishgray or brownish-gray sticky mass. The tears, when hard, hreak with a conchoidal fracture, showing a milk-white color, which changes gradually on expo- 
sure to pink, and finally to brown. It has a persistent, alliaceous odor, and bitter, alliaceous, acrid taste; when triturated with water, it yields a milkwhite emulsion. It is partly soluble in ether, and at least sixty per cent. of it should dissolve in alcohol. Dose: H., 3 ij.-iv.; D., gr. x.-xx.

Tinctura Asafoetidce, tincture of asatœtida (twenty per cent.). Dose: H., fl. 3 i.-iij.; D., fl. 3 ss.-ij.

Emulsum Asafoetidoe, emulsion of asafœetida (four per cent. in water). Dose: H., fl. 3 viij.-xx.; D., fl. $\frac{7}{3}$ ss. $-\mathrm{ij}$.

Physiological Action.-Asafœetida is a powerful antispasmodic, a carminative, a stimulating expectorant, a nerve and cerebral stimulant. It is also mildly tonic, laxative, diuretic, diaphoretic, emmenagogue, aphrodisiac, and anthelmintic. It raises arterial tension, stimulates the circulation, secretions, and excretions, the general nervous system, the menstrual flow, and sexual appetite. Its continued use at times causes impaired digestion, flatulency, difficult urination, diarrhœa, tenesmus, and phenomena of general nervousness. It is rapidly absorbed and eliminated by the respiratory mucous membrane, the kidneys, and the skin, gently stimulating their secretions.

TheraPeUtics. - It has long been successfully given in flatulent colic of the lower animals; in constipation-with aloes and nux vomica; in chronic bronchial affections, chronic catarrh-combined with ammonia muriate. Also as a vermifuge, but for this purpose its action is somewhat uncertain. 


\section{Cannabis Indica-Indian Hemp.}

The flowering tops of the female plant of Cannabis sativa (natural order Urticaceæ, grown in the East Indies. Sometimes known as foreign Indian hemp, in contradistinction to Cannabis Americana, the same plant grown in the Southern United States, but not nearly so powerful in action as the East Indian variety. Indian hemp contains a resin named cannabin, and a volatile oil from which are obtained cannabene, a light hydrocarbon, and cannabene hydride, a crystalline body.

Preparations.-Extractum Cannabis Indica, solid extract of Indian hemp. Dose: H., 3 ss.-i.; D., gr. $\frac{1}{4}-1$.

Extractum Cannabis Indicoe Fluidum, fluid extract of Indian hemp. Dose: H., fl. 3 ij.-iv.; D., mi. $-\mathrm{x}$.

Physiological Action.-Antispasmodic, anæs- . thetic, anodyne, analgesic, narcotic, cerebro-spinal stimulant, aphrodisiac. It increases motor and intellectual activity, stimulates vasomotor nerves, raises arterial tension, and depresses sensation. Has been given in exceedingly large doses, and although it produced great temporary mental excitement followed by coma, it is said never to have caused death.

TheraPeUtics.-In colic, to relieve spasm and pain, without interfering with the peristaltic movement; traumatic tetanus-large doses (Dr. R. Rutherford, of Edinburgh, claims to have cured over fifty 
per cent. of all tetanus patients by this method); chorea of the dog, to control the involuntary spasms; to dull sensibility during the performance of minor operations; in uterine affections-as metritis, cystitis, etc. -internally and locally by injection; in retention of urine, spasm of sphincter vesicæ, and dysuria; also in azoturia, to control excitement.

Cocainfe Hydrochloras-Hydrochlorate OF Cocaine.

A crystalline alkaloid, obtained from the leaves of Erythroxylon coca, a small Peruvian shrub (natural order Erythroxylaceæ), in which it is contained to the amount of about twenty-six per cent. "The hydrochlorate occurs as small whitish or almost colorless acicular crystals, or crystalline powder, is readily soluble in 0.5 part water and in $3 \frac{1}{2}$ parts of alcohol, has a bitter taste, producing a tingling sensation on the tongue followed by numbness. It paralyzes the sensory nerves with which it comes in contact, hence is used as a local anæsthetic. It has also slight antiseptic powers. In small doses it is a cerebral, cardiac, respiratory, and nerve stimulant and diuretic, but lessens the quantity of urea excreted, since it partly suspends the processes of waste, thereby acting as an indirect nutrient and enabling the body to maintain its energy on a decreased food supply. It dilates the pupils when used locally as well as systemically. Over-doses destroy life by paralysis of respiration.

TherapeUtics. - It is in general use as a local anæsthetic during minor surgical operations, as on 
the eye, larynx, in neurotomy, etc.; also to detect obscure lameness. As a mydriatic it is much more serviceable than atropine. Has been given in convalescence from fevers and protracted illness, in doses of grs. i.-v. to the horse; for the dog, gr. $\frac{1}{8}-1$. For local anæsthetic effect upon the horse, inject hypodermically from gr. $\mathrm{v} .-\mathrm{xv}$. occasionally more is required. Its effect begins in about twenty minutes and continues for from one-half to two hours; maximum, in about one hour. If kept in solution, add $\frac{1}{200}$ part of boric acid to prevent decomposition.

\section{Caffeina-Caffeine; Theina-Theine.}

Caffeine and theine are now generally conceded to be identical; the former is obtained from the dry seeds of Coffea Arabica (the coffee tree), the latter from the dry leaves of Thea sinensis (the tea plant). They are also found in other plants. Caffeine occurs as white, shining, elastic needles, soluble in 80 parts of water, 50 parts of alcohol, and in 9 parts of chloroform. It is a cardiac tonic and diuretic, and is used as a substitute for digitalis, which it occasionally effectually replaces. It is in medicinal doses also a stimulant to the nerve centres in the cerebrum, medulla, and cord; but over-doses paralyze. It regulates the heart's action, and causes the removal of œdema by increased diuresis. It is rapidly absorbed, rapidly eliminated, and has no cumulative effects. It is best given hypodermically with sodium salicylate, which increases its solubility and activity. Dose: H., gr. v. $-\mathrm{xx}$; D., gr. $\frac{1}{4}-2$. 


\section{CEREBRAL DEPRESSANTS.}

\section{Alcohol and Its Preparations.}

An alcohol may be defined as being a volatile organic compound of an organic radical and hydroxyl ( $\mathrm{HO}$ ), containing no $\mathrm{N}$, having great affinity for water, and reacting with acids to form $\mathrm{H}_{2} \mathrm{O}$ and ethers. An alcohol is obtained by distillation from the fermented product of grape sugar or other substance, such as starch, which may be readily converted into grape sugar. This fermented product, in the presence and by the growth of certain low vegetable organisms (the yeast plant, etc.), splits up into alcohol and $\mathrm{CO}_{2}$. The most important alcohols are:

1. Methylic Alcohol- $\mathrm{CH}_{4} \mathrm{O}-$ methyl hydrate, wood spirit.

2. Ethylic Alcohol- $\mathrm{C}_{2} \mathrm{H}_{6} \mathrm{O}$-ethyl hydrate, grain spirit.

3. Amylic Alcohol- $\mathrm{C}_{5} \mathrm{H}_{12} \mathrm{O}$-amyl bydrate, potato spirit, fusel oil.

When speaking of "alcohol" medicinally or pharmaceutically, the ethyl hydrate or grain spirit is the one referred to (see Alcohol or Rectified Spirit below).

The Official Preparations of Alcohol are: Alcohol Absolutum, spiritus absolutus, absolute alcohol, is ethyl alcohol containing not more than one per cent. by weight of water. It is a mobile, colorless, volatile liquid, having a spirituous odor and an intensely burning taste. Specific gravity, 0.797 at $60^{\circ} \mathrm{F}$. 
Alcohol, alcohol. Contains about ninety-one per cent. by weight, or ninety-four per cent. by volume, of ethylic alcohol, but no fusel oil. It is a transparent, colorless, mobile, and volatile liquid, of a characteristic, pungent, and agreeable odor, a burning taste, a neutral reaction, is very inflammable, burns with a smokeless blue flame, and has a specific gravity of 0.820 at $60^{\circ} \mathrm{F}$. It should be kept in wellstoppered glass vessels remote from lights and fire. Dose: H., fl. z i.-ij.; D., fl. 3 i.-ij.

Alcohol Dilutum, diluted alcohol (45.5 per cent. by weight [fifty-three per cent. by volume] of ethyl alcohol; and 54.5 per cent. by weight [forty-seven per cent. by volume] of water. Specific gravity, 0.928 at $60^{\circ} \mathrm{F}$.

Spiritus Frumenti, whiskey. Obtained by the distillation of the mash of fermented grain; from rye, wheat, or corn (United States), barley (Scotch), and at least two years old. (Irish whiskey is obtained from potatoes.) Contains from forty-four to fifty per cent. by weight of alcohol; also contains ethers developed by the action of butyric and acetic acids on the alcohol, and traces of fusel oil. Dose: H., fl. 亏 ij.-viij.; D., fl. 3 .i-fl. $z$ ij. (always well diluted). Spiritus Vini Gallici, brandy. Obtained by the distillation of fermented grape juice, and at least four years old. Contains from thirty-nine to forty-seven per cent. by weight of alcohol, and certain ethers which are developed by age. If pale it is colored from the cask; if dark it is colored by the addition of caramel or burnt sugar. Brandy is frequently 
prepared artificially by adding to high wine (alcohol) acetic or nitric ether, caramel, and logwood or catechu for astringency. Dose same as of whiskey.

Vinum Album, white wine. Contains ten to fourteen per cent. by weight of alcohol. Prepared by fermenting the unmodified grape juice (freed from skins, seeds, and stems).

Vinum Album Fortius, stronger white wine. Contains twenty to twenty-five per cent. by weight of alcohol. (Vinum album $\%$, alcohol 1).

Vinum Rubrum, red wine. Contains from ten to fourteen per cent. by weight of alcohol. It is made by fermenting the juice of colored grapes with their skins.

Unofficial Alcoholic Preparations.-Proof Spirit. Contains forty-nine per cent. by weight of absolute alcohol, with a peculiar oil and other foreign matters; specific gravity, 0.920.

Rectified Spirit. Contains eighty-five per cent. by weight of absolute alcohol and no fusel oil; specific gravity, 0.835 .

Rum (Jamica and St. Croix). From the distillation of fermented molasses.

Gin. Distilled from rye or barley, and flavored with juniper berries and hops. Cheaper grades are sometimes flavored with oil of turpentine.

Vinum Portense, port wine. Contains from thirty to forty per cent. of alcohol.

Vinum Xericum, sherry, contains from twenty to thirty-five per cent. of alcohol.

Beer contains alcohol two to three per cent.; Ale 
contains alcohol two to six per cent.; Porter and Stout contain alcohol four to six per cent.; also extract of malt- $\mathrm{CO}_{2}$-lactic acid, salts of $\mathrm{K}$ and $\mathrm{Na}$, aromatics, etc.

Physiological Actions of Alcohol.- It is a cerebral excitant and depressant, and a narcotic poison; also anæsthetic, antiseptic, antiparasitic, antispasmodic, antipyretic, a mild counter-irritant; coagulates albumen by abstracting its water; has also slight astringent effects. It is very diffusible, and when ingested becomes partly oxidized by the organism, and is partly excreted. Small doses relax the vessels, stimulate the gastric glands, promote the appetite and digestion, decrease the elimination of waste products (urea and $\mathrm{CO}_{2}$ ), cause a slight sensation of heat, and a slight rise of the body temperature. Being also a diffusible stimulant, it briefly stimulates the heart, increasing the functional activity of all organs, partly the kidneys, which excrete the unassimilated portion of the drug. The longcontinued administration of moderate or even small doses causes congestion of the stomach and liver, impairs the oxidizing power of the blood, and causes indigestion and gastric catarrh. Large doses precipitate pepsin and destroy its activity as a ferment, arrest digestion, produce exhilaration, intoxication, delirium, muscular incoördination, depressed heart, lowered arterial tension and bodily temperature, abolishment of reflexes, and lastly coma. Toxic doses cause a primary period of excitement, then insensibility, stertorous breathing, dilated or contracted pupils, 
complete muscular resolution, and death by paralysis of respiration and heart.

Treatment of Acute Alcoholism.-Evacuate the stomach if possible; inhalation of ammonia vapor; cold affusion to head and neck, warmth and friction to the extremities; faradization of respiratory muscles; mucilaginous drinks. Ammonium chloride given in solution is said to restore the faculties and to antagonize the stupor.

Therapeutics.-Alcohol, besides being used extensively as a solvent for many drugs in pharmacy and the arts, is sometimes given as a food, but only a very small quantity is used up in the system, and its continued use is certainly deleterious. It is given in atonic indigestion, in small doses, to stimulate gastric secretions and improve the appetite; in both flatulent and spasmodic colic, to expel flatus and to relieve spasms. In diarrhœa, combined with sodium benzoate and vegetable astringents; cardiac failure or weak heart from fright, chill, or shock; in milk fever of cows, with strychnine, belladonna, and sweet spirit of nitre; in epizootic catarrh and sore throat, when heart is weak and respirations quick and hurriedcombine with spiritus ætheris nitrosi and camphor, given in linseed tea; in pneumonia and other diseases of the respiratory apparatus, it is frequently conjoined with quinine sulphate and tincture of aconite. It is given in pyæmia, septicæmia, and in convalescence from all debilitating disorders; also immediately before chloroform anæsthesia to sustain the heart and prolong the chloroform narcosis. Exter- 
nally it is rubbed into the skin as a mild counter-irritant, rubefacient, and stimulant. Weak solutions with carbolic or salicylic acids are applied to relieve the itching of various skin diseases, as antiseptics to wounds, bedsores, and to check hemorrhage. An excellent refrigerant lotion is composed of alcohol $\xi$ iv, acetic acid $\xi$ iss., Goulard's extract $\xi$ ss., water $\xi$ xij. As a nutrient tonic and stimulant, hot ale or beer is frequently given in convalescence from exhausting diseases; to the horse and cattle in doses of O i.-ij., three times daily.

\section{Ather-Ether.}

The light compound commonly known as "sulphuric ether," or "ether," is, correctly speaking, ethylic ether or ethyl oxide- $\mathrm{C}_{4} \mathrm{H}_{30} \mathrm{O}$-and is prepared by distilling ethyl hydrate or ethylic alcohol with sulphuric acid. It is purified by treating with quicklime and redistillation.

Ather, ether, commercial ether, is a liquid composed of about seventy-four per cent. of ethyl oxide and about twenty-six per cent. of alcohol, containing a little water. Specific gravity about 0.750 at $59^{\circ} \mathrm{F}$. Must be kept in well-stoppered bottles or in soldered tins in a cool place, remote from lights and fire. For properties of ether, see under Ather Fortior. It is soluble in five times its volume of water. Used chiefly externally.

Ather Fortior, stronger ether, a liquid composed of about ninety-four per cent. of ethyl oxide, 
and about six per cent. of alcohol, containing a little water. Specific gravity not higher than 0.725 at $59^{\circ} \mathrm{F}$. Like ether, it must be carefully preserved. It is a thin, clear, very diffusive and colorless liquid, of a refreshing, characteristic odor, a burning and sweetish taste, with a slightly bitter after-taste and a neutral reaction. It is soluble in all proportions, in alcohol, chloroform, benzol, benzine, fixed and volatile oils, and dissolves in eight times its volume of water at $59^{\circ} \mathrm{F}$. Ether is highly inflammable, and its vapor when mixed with air and ignited explodes violently. It is a solvent for oils, fats, resins, guncotton, gutta-percha, and other substances. Dose: H., fl. z i.-ij.; D., fl. 3 ss.-i. (always well diluted with cold water, gruel, oil, or linseed tea). For general anæsthesia.-Dose (by inhalation): H., fl. z iij.-viij.; D., fl. 3 ij.-vi. This is the ether which should be given internally and by inhalation.

Preparations of Ether.-Spiritus AEtheris Compositus, compound spirit of ether, Hoffmann's anodyne. Contains alcohol $6 \%$, stronger ether 30 , ethereal oil 3 parts.

Collodium, collodion. Contains pyroxylin 4, stronger ether 70, and alcohol 26 parts. Local use.

Collodium Flexile, flexible collodion. Contains collodion 92, Canada turpentine 5, and castor oil 3 parts. Local use.

Collodium Stypticum, styptic collodion. Contains tannic acid 20, alcohol 5, stronger ether 20 , and collodion 55 parts. Local use.

Spiritus AEtheris Nitrosi, Spiritus Nitrico- 
Dulcis, spirit of nitrous ether, sweet spirit of nitre. An alcoholic solution of ethyl nitrite, containing five per cent. of the crude ether. Dose: H., fl. z i.-iij.; D., Mxv.-fl. 3 i.

Physiological Action.-Ether is a cardiac and cerebral stimulant, anodyne, antispasmodic, diaphoretic, anthelmintic, anæsthetic, and a narcotic poison. Given internally, it is one of the most powerful stimulants to the secretions, especially to the salivary glands, stomach, and pancreas. It is quickly absorbed from the stomach and intestines, but still more rapidly if introduced as a vapor into the lungs by inhalation, and acts very quickly upon the central nervous system, nerve centres, and nerve endings. Small doses stimulate; larger doses, after a brief period of stimulation, depress, paralyze, and anæsthetize the centres of the brain and cord. It is eliminated by all the secretory channels, stimulating them, and in this way exerts its expectorant, diaphoretic, and diuretic actions; but the elimination for the most part is accomplished by the lungs. If the vapor is inhaled there is at first a slight irritation to the fauces, sometimes causing cough, a feeling of strangulation or constriction of the throat, and more or less cerebral excitement or intoxication, during which time the pulse and respirations are quickened. This is followed by a period of slight tetanic convulsions, when the muscles become rigid and the breathing stertorous. This condition rapidly subsides, complete insensibility is established, the muscles are relaxed, reflexes are abolished, the cerebral functions are en- 
tirely suspended, and the processes of respiration and circulation are carried on only by the lower centres in the medulla. If the inhalations of the ether vapor are still continued after this, these lower centres also become paralyzed, and death takes place by respiratory arrest, the heart usually continuing its pulsations for a long time after respiration has ceased (chloroform usually paralyzes the heart). In order to produce general anæsthesia, the ether vapor should be inhaled in a fairly concentrated form for from three to ten minutes. Hypodermic injections of atropine antagonize the toxic effects of ether more effectually than any other known drug.

TheraPeUtics.-Ether alone or combined with chloroform and alcohol in varying proportions as a general anæsthetic. Ether spray as a local anæs. thetic; internally - as an antispasmodic in both flatulent and spasmodic colic, combined with other anodynes and carminatives. Enemata of ether to allay abdominal irritation; also to destroy rectal worms. The same treatment is much favored by French veterinarians in intussusception. In hepatic colic-ether combined with turpentine, to act as a solvent for the hepatic calculi; as a prompt and powerful stimulant in syncope or collapse-if from exhausting disease, shock, or hemorrhage. In puerperal apoplexy in cattle it is combined with alcohol or whiskey, and when the patients are unable to swallow it may be given subcutaneously. In convalescence from exhausting disease-combined with aromatics and bitters, improves the appetite, strengthens the enfeebled 
pulse, and also tends to relieve cough and other irritability.

For special instructions on the use of ether as a general anæsthetic, see under Chloroform. Local anæsthesia, sufficient for performing short operations, such as inserting setons, opening abscesses or fistulæ, tendenotomy, neurotomy, etc., may be produced by ether spray to the part, the amount required being from one to one and one-half ounces; but for these purposes cocaine is now generally preferred. It may also be injected hypodermically when immediate effects are required. Externally ether is sometimes combined with liniments as an anodyne and stimulant, but must be carefully employed on account of its highly inflammable nature.

Spiritus ætheris nitrosi is a carminative, antispasmodic, general stimulant, a very good diaphoretic and diuretic, and is extensively used in influenza, various fevers, pneumonia, bronchitis, asthma, colic. It is excreted chiefly by the kidneys and skin; hence its diaphoretic and diuretic actions are very marked. It should be given always well diluted.

\section{ChLoroformuM-ChLOROFORM.}

Chloroform, or methyl terchloride- $\mathrm{CHCl}_{3}$-is prepared by the action of chlorinated lime upon alcohol, is purified (1) by repeated agitation with water, which washes away saline, acid, and some organic impurities; (2) shaking with sulphuric acid (free from nitric acid), which chars and removes the last 
trace of organic oils; (3) admixture with slaked lime and calcium chloride to neutralize acidity and take up the water; (4) by very careful distillation. Its discovery is claimed by Samuel Guthrie, of Sacketts Harbor, 1831, and also jointly by Liebig and Soubeiran, 1832.

Chloroformum Purificatum, purified chloroform, is a heavy, clear, colorless, diffusive, practically noninflammable liquid, of a characteristic, pleasant, ethereal odor, a burning, sweet taste, and a neutral reaction. Soluble in 200 parts of water and in all proportions in alcohol or ether; also in benzol, benzin, fixed or volatile oils. Specific gravity, 1.485 . Its vapor is four times heavier than air, and it is a solvent for caoutchouc, gutta-percha, fats, resins, balsams, oils, wax, iodine bromine, and most of the alkaloids. Dose: H., fl. 3 i.-ij.; D., $m$ v.-x.-for internal medication. Inhalation for anæsthesia-Dose: H., fl. 亏亏 i.-ij.; D., fl. 3 i.-iv.

Cloroformum Venale, commercial chloroform. A liquid containing not less than ninety-eight per cent. of chloroform, having similar properties to the former preparation. Specific gravity, 1.4\%. For local use only, as it is not pure enough for any other purpose.

Linimentum Chloroformi, chloroform liniment. For local use only, is composed of chloroformum venale 40 and linimentum saponis 60 parts.

Linimentum Chloroformi Compositum, compound chloroform liniment, a very excellent anodyne application, is composed of chloroform venale 1 , tur- 
pentine 1 , laudanum $\frac{1}{2}$, tincture aconite root $\frac{1}{4}$, and soap liniment 2 parts by measure.

Spiritus Chloroformi, spirit of chloroform, socalled chloric ether (æther chloricus). Contains purified chloroform 10 and alcohol 90 parts. Dose: H., fl. 3 i.-ij.; D., fl. 3 i.-iss.

Ancesthetic Mixtures contain chloroform, ether, with or without alcohol, in varying proportions. The best known and most serviceable are:

A. C. E., 1, 2, 3 mixture, containing alcohol 1 , chloroform purified 2 , ether 3 .

E. C. A., 3, 1, 1, Nussbaum's mixture, containing ether 3, chloroform 1, and alcohol 1 part.

Physiological Actions of Chloroform.-Small doses given internally are carminative, antispasmodic, and analgesic. Large doses cause muscular relaxation and paralyze the cerebro-spinal nervous system. Death takes place by paralysis of both heart and respiration, similar to ether, but its toxic action is very much more rapid than that of ether; hence death by chloroform is more sudden. Applied locally, chloroform is a rubefacient, a refrigerant, anodyne, and local anæsthetic.

General anæsthesia is produced by an inhalation of the vapor, and shows four distinct stages, viz.: (1) Stimulant-some excitement and struggling; (2) narcotic-muscular relaxation, quietude, and stupor; (3) anæsthetic-complete anæsthesia, insensibility; (4) paralytic-death ensuing if the action of the drug is continued still further.

In order to avoid heart failure or depression, it is 
a very good plan to inject hypodermically a small dose of morphine and atropine fifteen to twenty minutes prior to placing the animal under the action of an anæsthetic. Chloroform as a rule is given with the admission of a considerable volume of air, but some prefer to allow it to be inhaled without air, claiming that under these circumstances a much less quantity is required, and hence all dangers are correspondingly decreased. During anæsthesia the operator must carefully watch both respirations and pulse, allowing full draughts of pure air as soon as complete anæsthesia is produced; occasionally gently touching the cornea with the finger to note if reflex excitabil. ity is returning; if so, administer more chloroform. Never operate on any animal unless under complete anæsthesia, as the incomplete stage is very dangerous, since in this condition reflex inhibition over the pneumogastric upon the heart may be the means of arresting the action of the heart's motor ganglia.

Contra-Indications for Ancesthesia are dilatation of the heart, fatty degeneration of the heart, kidney disease, emphysema of the lungs, and brain tumors.

Treatment of dangerous symptoms while under the action of chloroform anæsthesia are: Artificial respiration if possible, draw tongue forward and clear away mucus from mouth, warm applications and friction to body and limbs, and hypodermic injection of atropine.

Since chloroform in its purity is irritant to the 
mucous membranes, it must always be given well diluted, else it may cause a violent gastro-enteritis.

Chloroform is much used in obstetrical practice to control spasms and violent uterine throes when trying to rectify false presentations; also to relax rigidity of the os in delayed parturition, and to relieve after-pains.

Inhalations of chloroform are frequently giren to relax and relieve intestinal spasms; also in the reduction of intestinal hernia; to relieve the spasm of tetanus; to check the fits of chorea and epilepsy in dogs; to relieve spasmodic cough. Externally - as a local anæsthetic, the same as cocaine; in liniments, to rheumatic and painful swellings and joints.

\section{Chloral-Chloral (Chloral Hydrate).}

Chloral itself is trichloraldehyde- $\left(\mathrm{C}_{2} \mathrm{HCl}_{3} \mathrm{O}\right)$-an unstable, oily, colorless fluid, formed by the action of chlorine upon alcohol. Its hydrate, the official chloral- $\left(\mathrm{C}_{2} \mathrm{HCl}_{3} \mathrm{O}+\mathrm{H}_{2} \mathrm{O}\right)$-is seen as separate, rhomboidal, colorless, transparent crystals, slowly evaporating on exposure to air, having an aromatic, penetrating, and slightly acrid odor, a bitterish, caustic taste, soluble in less than its own weight of water, in 4 parts of chloroform; also in alcohol, ether, glycerin, fixed and volatile oils. Its aqueous solution soon acquires an acid reaction, but its alcoholic solution remains neutral. It liquefies when rubbed with carbolic acid or with camphor, and is readily decomposed by alkalies into chloroform and a formiate of the alkaline 
base. Chloral was discovered by Baron Liebig in 1832, but was not employed in medicine till 1869 . It is prepared by passing chlorine gas through alcohol until saturated, then purified by distillation with $\mathrm{H}_{2} \mathrm{SO}_{4}$, and finally mixed with $\mathrm{H}_{2} \mathrm{O}$ to form the solid chloral hydrate. Dose: H., z i.-ij.; D., gr. v.-xx.

Physiological ACtion.-Chloral is a powerful depressant of the cerebro-spinal centres, a good hypnotic and calmative, an antispasmodic, antiseptic, antiferment, and counter-irritant. Compared with chloroform it is more hypnotic but less anæsthetic. It cannot be used subcutaneously, as it is too irritant and would cause extensive sloughing of the skin at the point of injection. Large or concentrated doses may cause severe gastritis, while in the smaller animals nausea and vomiting are frequently produced. Chloral has a selective action on the brain, causing a cerebral anæmia, which produces a very normal-like sleep, and from which the animal may be readily awakened, but shortly after falls into, until the hypnotic action of the chloral is exhausted. An important point in chloral narcosis is that it but seldom leaves any bad after-effects or serious depression, although this is more noticeable in animals in which instead of producing sleep it has caused delirium and excitement. Full doses, when first given, briefly stimulate the heart, but soon this vital organ becomes depressed, arterial tension is lowered, oxidation is diminished, and bodily temperature lowered. Large doses rapidly cause a profound narcotism, abolishment of the reflexes and sensibility, complete muscu- 
lar relaxation, and a very great fall in temperature. Toxic doses cause death from paralysis of the cardiae motor ganglia and the respiratory centre, or by sudden heart failure in fatty degeneration, the heart being arrested in diastole with the right cavities distended; hence it must be very carefully used on patients affected with heart trouble of any kind or advanced pulmonary disease.

Chloral is not a true anodyne, as it neither interrupts the transmission of pain nor does it affect the conductivity of the sensory nerves, but overwhelms the brain centres, thus rendering the animal unconscious of pain; and hence it may be called an indirect anæsthetic. Chloral is rapidly diffused in the blood, being supposed to set free chloroform due to its decomposition by the blood, which is an alkaline fluid. It increases the fluidity of that tissue, crenates the red corpuscles, in large quantity destroys the leucocytes, prevents the coagulation of fibrin, and produces a general anæmic condition. It is excreted, partly unchanged, by the kidneys, causing some diuresis, and also by the skin.

Treatment of Chloral Poisoning.-Atropine in small frequently repeated doses is the best antagonist, as it counteracts the depression of the chloral on the heart, respiration, and cerebro-spinal system. Morphine is sometimes given with chloral to prevent the tendency to cardiac failure.

Therapeutics. - Chloral is given to quiet irritability and to cause sleep, to relieve gastro-intestinal irritation and spasm-as in colic. But as it is a topi- 
cal irritant, it should not be given if there is congestion or inflammation; in fevers attended with excitement and restlessness; in spasmodic cough in all animals; in canine asthma and distemper; conjoined with bromides and cannabis indica in tetanus; as an antagonist in strychnine poisoning; in parturient apoplexy of cattle, attended with nervous excitement; in phrenitis, chorea, and epilepsy. In obstetrics-to relieve pain, to relax the os uteri, to palliate puerperal convulsions, and to relieve after-pains. Externallya twenty-five per cent. solution is used locally as an antiseptic and anodyne to cancers and ulcers; applications of chloral-camphor to relieve pain and irritability of neuralgic affections, and the itching attendant upon many skin troubles. Chloral is always best given per os and in well-diluted solution.

\section{OPIUM-OPIUM.}

The concrete, milky exudation, obtained by incising the unripe capsules of Papaver somniferum, or white poppy, an annual herb, natural order Papaveraceæ, habitat Asia Minor. In its normal, moist condition it should yield not less than nine per cent. of morphine, when assayed by the official process. It contains seventeen alkaloids, two neutral bodies, two organic acids; also wax, gum, sugar, resin, extractives, odorous principles, etc. The six principal alkaloids of opium are:

1. Morphina, morphine-hypnotic, anodyne, and narcotic. 
2. Codeina, codeine-calmative and less constipating.

3. Thebaina, thebaine-a tetanizer and not used medicinally.

4. Narceina, narceine-regarded as the most hypnotic of the six.

5. Papaverina, papaverine-of doubtful action; supposedly narcotic and convulsant.

6. Narcotina, narcotine-wrongly named, as it is a tetanizer and highly antiperiodic.

These principles are combined in the plant with meconic and lactic acids.

A derivative of morphine, obtained by the action of $\mathrm{HCl}$, is-

Apomorphina, apomorphine-an artificial alkaloid and a powerful emetic. Its hydrochlorate, which is official, is given to the dog in doses of gr. $\frac{1}{8}$ per os, or gr. $\frac{1}{20} \frac{1}{16}$ subcutaneously.

Chief Preparations of Opium used in veterinary practice are:

Opii Pulvis, powdered opium. Dose: H., 3 i.-ij. ; D., gr. $\frac{1}{4}$-iij.

Extractum Opii, extract of opium (aqueous). Dose: H., 3 ss.-i. ; D., gr. $\frac{1}{8}$-iss.

Tinctura Opii, U.S. P. (ten per cent.), tincture of opium, laudanum. Dose: H., fl. z i.-ij.; D., Tl viij. $-\mathrm{xx}$.

Tinctura Opii Camphorata, Elixir Paregoric, camphorated tincture of opium, paregoric, elixir paregoric. Contains powdered opium 4, benzoic acid 4, camphor 4, oil of anise 4, glycerin 40, and diluted al- 
cohol enough to make 1,000 parts. Dose: D., fl. 3 ss.iv.

Pulvis Ipecacuanhoe et Opii, Dover's powder. Contains ipecac 1, opium 1, sugar of milk 8 parts, triturated to a fine powder. Dose: H., 3 i.-iij.; D., gr. v. - $\mathrm{xv}$.

Preparations of Morphine, ETC.-Morphince Acetas, acetate of morphine; Morphince Hydrochloras, hydrochlorate or muriate of morphine; Morphince Sulphas, sulphate of morphine. Dose: H., gr. iij.-X.; D., gr. $\frac{1}{15} \frac{1}{4}$; subcutaneously one-half of these doses.

Morphina sulphas occurs as white, feathery, acicular crystals, of a silky lustre, permanent in the air, odorless, having a bitter taste and a neutral reaction. Soluble in 24 parts of water, in 702 parts of alcohol, and in 0.75 part of boiling water.

Liquor Morphince Sulphatis Magendi, Magendie's solution of morphine. Has morphine sulphate grs. xvi. dissolved in distilled water 1 ounce.

Liquor Morphince Sulphatis, U. S. P., 1870. Has morphine sulphate gr. i. to the ounce of distilled water. (Special care must be taken to distinguish between these two solutions.)

Codeina, codeine. White or yellowish-white, more or less translucent, rhombic prisms, somewhat efflorescent in warm air, odorless, having a slightly bitter taste and an alkaline reaction. Soluble in 80 parts of water, in 17 parts of boiling water, in 6 parts of ether, and very soluble in alcohol and in chloroform. Dose: H., gr. v.-xx.; D., gr. $\frac{1}{4}-\mathrm{ij}$. 
Codeince Phosphas, phosphate of codeine. Is given in the same doses as the preceding, but is much more soluble (1:20 of water).

Tests for Morphine. - Nitric acid produces a bloodred, turning orange, then yellow, then disappearing. Ferric chloride gives a rich blue with morphine; a dark-brown with meconic acid or any preparation of opium. Iodic acid liberates iodine, which may be tested for with starch.

Physiological Action.-Opium and its preparations are stimulant to the heart and brain, hypnotic, antispasmodic, deliriant, narcotic, sedative, myotic, and diaphoretic. These last two are not so prominent in the lower animals as in man. Small doses increase the heart's action, raise arterial tension, stimulate respiration, contract the pupils, and while at first slightly stimulating the glandular intestinal apparatus, soon check all secretions except sweat and milk. Full doses intensify the heart's action, cause more or less stertorous and shallow respiration, itchiness of the nose, retention of urine, pulse becoming gradually irregular; some diaphoresis. In some animals a deep sleep, in others delirium and excitement. Larger doses cause a weak and slow pulse, very irregular and stertorous respirations, a cold clammy sweat, and coma.

ToXICOLOGY.-Death takes place from paralysis of the respiratory centre. Experiments on the lower animals by the writer showed that they are relatively not as much affected by opium and its preparations as man. The horse more usually becomes restless 
excited, and delirious than relapsing into a sleep or coma; the same is true of the bovine species. Again, in both of these animals the pupil does not always respond to the action of the drug, neither is diaphoresis so readily induced by it as in man. The dog, however, is more susceptible to this drug than the equine or bovine, and although after a brief period of excitement these animals are overcome by its action, they are quite readily awakened. Particular individuals of all these species, however, will be found quite susceptible to the action of opium and its preparations. The writer injected subcutaneously morphine sulphate $3 \mathrm{i}$. in a mare of one thousand pounds at 9 P.M. In fifteen minutes the animal appeared slightly drowsy, but rapidly became nervous and excited; by $9: 30$ P.M. the pulse was increased both in force and volume, respirations slightly stertorous. 10 P.M : Forty-five grains more were injected; pulse continued, strong and bounding, increased nervous excitement, pupils somewhat dilated. 12 P.M.: The animal appeared still in about the same condition, and at 3 A.M. gr. 1x. more were injected; the pulse was now somewhat weaker than before and respirations irregular and laborious. 6 A.M. : The last symptoms aggravated, pulse very weak and irregular, nervous excitement much abated, insensibility to pain. 8.30 A.M. : Great dulness, cold sweat, pulse very small and weak, respiration jerky and irregular, pupils contracted. Death took place at 9 A.M. Post-mortem examination showed a wet brain, congested lungs, blood fluid and dark-colored, engorgement of the 
venous trunks and right side of heart, bladder distended with a dark-colored urine.

Treatment of Opium Poisoning.-The stomach should be evacuated whenever practicable, respiration and circulation maintained; intratracheal or subcutaneous injections of atropine in small doses (carefully, lest atropine narcosis be substituted for the opium narcosis). This is one of the most complete antagonists known. The animal should if possible be kept in motion, and the bladder frequently evacuated to prevent reabsorption.

Permanganate of potash is also a very excellent antidote. The writer found that an aged gelding poisoned by morphine sulphate $3 \mathrm{i}$. made a complete recovery in four hours, after receiving drachm doses of the permanganate every half-hour for six consecutive doses.

Caffeine, cocaine, and strychnine are also antagonistic to some of the effects of opium.

THERAPEUTICS.-The chief indications for the use of opium or morphine are: (1) To relieve pain; (2) to produce sleep; (3) to allay irritation; (4) to check excessive secretions; (5) to support the system; (6) as a sudorific. It should be carefully used in very young animals or in old animals; also in advanced disease of the kidneys, respiratory apparatus, cardiac troubles, acute fevers, and obstinate constipation.

Opium and its preparations are given to relieve. pain from any cause except acute inflammation of the brain; in irritation of the bronchi, irritable cough, irritation of the bladder, stomach, intestinal appara- 
tus; in persistent vomiting due to irritation of the vomiting centre; in diarrhœa and dysentery-usually with tincture kino or other vegetable astringent; gastritis, enteritis, peritonitis; muscular rheumatism; spasms, spasmodic colic; in cerebro-spinal meningitis-to relieve pain and irritability; tetanus; in diabetes mellitus - to reduce the quantity of sugar; to relieve post-partum irritability and straining in mares, cows, and bitches; also to check premature labor pains. Externally-as a local anodyne and sedative, to relieve pain and irritability of wounds, bruises, sores, etc., see Liquor Plumbi et Opii and Plumbum; applications of laudanum and soap liniment to chest walls in pleurisy; painful uterine affections-douches of hot water and laudanum.

\section{Bromine AND Bromides.}

Bromum, bromine, is a liquid, non-metallic element, having the symbol $\mathrm{Br}$, and is obtained from sea-water and certain saline springs. It is a dark, reddish-brown, mobile liquid, evolving, even at ordinary temperature, a yellowish-red vapor highly irritating to the eyes and lungs, and having a peculiar, suffocating odor, resembling that of chlorine. It is soluble in 33 parts of water and very soluble in both ether and alcohol. It is rarely used medicinally except by inhalation and as an escharotic.

The Chief Preparations of Bromine used in veterinary practice are:

Potassii Bromidum, potassium bromide- $\mathrm{KBr}$. Colorless, translucent, cubical crystals, permanent in 
dry air, odorless, having a pungent, saline taste and a neutral reaction. Soluble in 1.6 parts of water and in 200 parts of alcohol.

Sodii Bromidum, sodium bromide-NaBr. Small, colorless or white, monoclinic prisms, or a crystalline powder, permanent in dry air, odorless, having a saline, slightly bitter taste and a neutral or faintly alkaline reaction. Soluble in 1.2 parts of water and in 13 parts of alcohol.

Lithii Bromidum, lithium bromide-LiBr. A white, granular salt, very deliquescent, odorless, having a very sharp, somewhat bitter taste and a neutral reaction. Very soluble both in water and in alcohol.

Ammoni Bromidum, ammonium bromide$\mathrm{NH}_{4} \mathrm{Br}$. Colorless, transparent, prismatic crystals, or a white granular salt, becoming yellow on long exposure to air, odorless, having a pungent, saline taste and a neutral reaction. Soluble in 1.5 parts of water and in 150 parts of alcohol.

Calcii Bromidum, calcium bromide-- $\mathrm{CaBr}_{2}$. A white, granular salt, very deliquescent, odorless, having a pungent, saline, and bitter taste and a neutral reaction. Soluble in 0.7 part of water and in 1 part of alcohol.

The actions of these bromides are very nearly identical, and their dose is as follows: H. and C., 3 iv.亏i.; D., gr. v. -xxx.

Camphora Monobromata.-See Camphor. Acidum Hydrobromicum Dilutum, diluted hydrobromic acid. A clear, colorless, aqueous liquid, con- 
taining ten per cent. of absolute $\mathrm{HBr}$ acid, and used as a substitute for the bromides. Dose: H. and C., fl. 3 ss.-ij.; D., $7 l$ xx.-fl. 3 ij.

Physiological Action of Bromine.-It is an active and painful escharotic, a deodorant and antiseptic, setting free ozone, while its vapor is a powerful irritant to the eyes and respiratory mucous membranes, causing cough, sneezing, and dyspnœa. Internally-it is an active, corrosive poison, causing violent gastritis, depression, and collapse.

Physiological action of the Bromides. - The bromides all have a very salty taste, are very diffusible, and are very slowly eliminated. They become decomposed in the blood, but are reformed at the points of elimination-the fauces, intestines, skin, and kidneys-where they sometimes cause much irritation, and if continued for some time indigestion and even gastric catarrh may result. The bromides are depressants of the cerebral and spinal functions; they are alterative, antispasmodic, and hypnotic, the potassium salt particularly in toxic doses being a cardiac and muscular paralyzant. They all reduce the number of the respirations, lower the number and force of the heart beats, and lower arterial tension by diminishing the calibre of the arterioles. The activity of the brain cells is diminished, producing a state of somnolence or drowsiness, and the sensibility of the peripheral nerves is so diminished that a species of anæsthesia of the skin and mucous membranes is produced. Motility and sexual functions are somewhat impaired. There is more or less emaciation, 
subnormal body-temperature, fetid breath, sluggish reflexes, and a lack of coordination of movement. These last symptoms, taken collectively, are termed "bromism," and are probably due to the sedative action of the bromides upon the sympathetic system, causing anæmia of the brain, spinal cord, sexual organs, and skin. Toxic doses destroy life by asphyxia.

Differences IN ACtion of the Bromides.Potassium Bromide (containing sixty-six per cent. of bromine) is the most toxic to the heart and muscular apparatus, and the least hypnotic.

Sodium Bromide (containing seventy-eight per cent. of bromine) is the least toxic, but the most hypnotic. It acts strongly upon the circulation, but is more readily borne by the stomach than the others.

Ammonium Bromide, very much resembling the potassium salt, has less influence upon the heart and muscular apparatus, but partakes of the stimulating properties of its base.

Lithium Bromide (containing ninety-two per cent. of bromine) is quite similar to the sodium salt, and is a very powerful hypnotic.

Calcium Bromide is not very active except as a hypnotic.

Incompatibles and Antagonists.-Incompatibles are acids, metallic salts, and nitrous ether. Vasomotor stimulants-as digitalis, ergot, and belladonna -antagonize many effects of the bromides, but morphine is the most complete antagonist.

Contra-Indications for the Use of the Bromides. -Anæmic conditions and weak or fatty heart. 
Therapeutics.-Cardiac excitement-the potassium bromide with digitalis. In epilepsy and chorea of dogs; in acute rheumatism-the $\mathrm{NH}_{4} \mathrm{Br}$ as an alkali; in muscular rheumatism-the LiBr. The bromides are given to relieve spasms of various kinds, to produce rest, quietude, and sleep-as in tetanus, colic, cerebral and spinal congestion, etc. In diabetes mellitus-the $\mathrm{NH}_{4} \mathrm{Br}$ with $\mathrm{As}_{2} \mathrm{O}_{3}$. The $\mathrm{HBr}$ acid is given in irritable cough, etc., and in all cases in which the bromides are indicated, except epilepsy.

\section{MOTOR DEPRESSANTS.}

\section{GeLsemium-Yellow JAsmine.}

The rhizome and rootlets of Gelsemium sempervirens, a climbing plant indigenous in the Southern United States, of the natural order Loganiaceæ, and containing an alkaloid known as gelsemine, in combination with gelsemic acid; also a resinous substance and a volatile oil.

Preparations.-Tinctura Gelsemii, tincture of yellow jasmine. Dose: H., fl. 3 ij.-viij.; D., $\pi$ x.fl. 3 i.

Extractum Gelsemii Fluidum, fluid extract of yellow jasmine. Dose: H., fl. 3 ss.+ij.; D., Tl ij.-xx.

Gelsemina, gelsemine. Dose: H., gr. $\frac{1}{4}-\frac{3}{4} ;$ D., gr. $\frac{1}{60}-\frac{T}{20}$.

This alkaloid is soluble in alcohol, ether, and chloroform, while its salts-the sulphate, nitrate, hydro- 
chlorate, and hydrobromate-are soluble in water and are given in the same doses.

Prysiological Actions.-Antispasmodic, diaphoretic, anaphrodisiac, and nervous depressant. In large doses paralyzing motility primarily, and sensibility by central action on the spinal cord, and frequently causing convulsions. Medium doses slow the heart rate and cause impaired sensibility, enfeebled muscular power, dilated pupils, and some diaphoresis. While gelsemium lowers the heart rate, it is not an arterial depressant and should not be given if the heart is weak. It does not irritate the gastrointestinal canal, and if given in small repeated doses lowers bodily temperature; the effect of a single medium dose continuing for about three hours. It is excreted chiefly by the kidneys and skin.

TheraPeUtICS.-In pneumonia and pleurisy, if heart is not yet weakened; in spasmodic cough; in remittent fever; in cerebro-spinal meningitis; in vesicular irritability, incontinence of urine, and dysmenorrhœa.

Antagonists are morphine, digitalis, ammonia, and alcohol, ranking in efficacy in the order named; but the application of heat to the body, artificial respiration, and emetics when possible, are of vital importance to counteract the toxic effects of the drug.

\section{Pilocarpus-J Jaborandi.}

Tie leaflets of Pilocarpus selloanus and of Pilocarpus jaborandi, Brazilian plants of the natural order Rutaceæ, containing an amorphous liquid alkaloid, 10 
pilocarpine, which is soluble in alcohol, ammonia, ether, chloroform, and dilute acids, forming crystallizable salts-the nitrate and hydrochlorate being chiefly used. Another alkaloid-jaborine-also occurs, but only in small quantity, and physiologically it is antagonistic to pilocarpine.

Preparations.-Extractum Pilocarpi Fluidum, fluid extract of pilocarpus. Dose: H., fl. 3 jj.-viij.; D., Tl x.-fl. 3 i.

Pilocarpince Hydrochloras, hydrochlorate of pilocarpine; Pilocarpince Nitras, nitrate of pilocarpine. Dose: H., gr. ij.-v.; D., gr. $\frac{1}{10-\frac{1}{6}}$; for subcutaneous injection.

Physiological Action.-Jaborandi stimulates peripheral nerve endings supplying involuntary muscular fibre and glands; is also a paralyzer of the vasomotor system, a diaphoretic, a sialogogue, and depresses the heart by stimulation of the vagus ends. Its myotic and emetic properties are not well marked on the lower animals, excepting the canine species. The same is true of its diaphoretic action, which in the dog is quite prominent, but on the horse and ox is quite uncertain and can only be induced if the animal be heavily clothed and given warm drinks along with full doses of the drug. The nasal, buccal, bronshial, and lachrymal secretions are very much increased, and sometimes a watery diarrhœa accompanies the administration of the drug, due to its powerful stimulant effect upon the muscular coat of the intestines as well as on the intestinal glandular apparatus. When first given, the cardiac action is 
increased, but afterward decreased, arterial tension is lowered, and bodily temperature falls from $1^{\circ}$ to $4^{\circ} \mathrm{F}$. The elimination of urea is greatly increased, but the amount of urine voided is not increased; the respiratory power is lowered, and apnœa may occur from accumulation of the increased mucous secretion. Pilocarpus is rapidly diffused and is eliminated by the skin and salivary glands, its effects continuing for from three to six hours. It causes contractions of the bladder, spleen, and uterus; hence must be cautiously used in pregnant animals.

Antagonists.-Morphine and strychnine counteract some of its effects, but atropine is the most complete antagonist.

TheraPeUtics.-Pilocarpus assists in the absorption of pleuritic and other serous effusions, is used in rheumatism, chronic eczema, and with other expectorants in catarrhal pneumonia; in inflammation of the brain, meningitis, laminitis, azoturia, erysipelas, and various dropsical affections; in torpidity of the bowel, obstinate constipation, and colic-combined with physostigmine; in diabetes insipidus-to reduce the quantity of the urine by throwing the eliminative work upon the skin; it also gives prompt relief in parotitis.

\section{Physostigma-Calabar Bean.}

(Faba Calabarica.)

The seeds of Physostigma venenosum, or ordeal plant, natural order Leguminosæ, a woody creeper of Calabar, West Africa. It contains, besides albu- 
minous matters, starch, oil, and an alkaloid, known as eserine, physostigmine, or calabrine (some writers claim a second alkaloid for the Calabar bean, and designate this as calabarine).

Chief Preparations.-Faba Calabarica, Calabar bean (powdered). Dose: H. and C., gr. xv.xxx. ; D., gr. $\frac{1}{4-\frac{1}{2}}$.

Physostigmince Salicylas, physostigmine (eserine) salicylate. Colorless, shining, acicular, or short columnar crystals, gradually turning reddish when long exposed to light and air, odorless, having a bitter taste and a neutral reaction. Soluble in 130 parts of water and in 12 parts of alcohol. The aqueous, also alcoholic solution, when exposed to light for a short time, turns reddish and should be discarded.

Physostigmince Sulphas, physostigmine (eserine) sulphate. In appearance and properties resembles the salicylate. Dose of either of these salts, subcutaneously or intratracheally: Dose: H., gr. i.-iss.; D., gr. $\frac{1}{40}-\frac{1}{20}$; per os, H., gr. ij.-iij.; D., gr. $\frac{1}{20-\frac{1}{10}}$.

Lamelloe Physostigmoe, disks of physostigmine, medicated disks. Used locally on the eye, each containing gr. $\frac{1}{1000}$.

Physiological Action.-Physostigma is a powerful muscular stimulant and direct spinal paralyzer, capable of producing a complete general paralysis and abolishment of the reflexes without affecting muscular irritability or the brain. Secretions are stimulated, nausea and vomiting are induced, particularly in carnivora. Its cathartic properties are 
brought about by its powerful stimulation of the intestinal walls, as well as by increasing the amount of intestinal secretion, thus rapidly causing the expulsion of gas and fæces (in the horse within fifteen to thirty minutes after intratracheal injection). The frequency of the heart beats are increased, but decreased in force, while arterial tension, though at first lowered, is raised. Over-doses cause dyspnœa by a tetanic action on the respiratory muscles, death taking place from $\mathrm{CO}_{2}$ poisoning induced by the paralysis of respiration. Physostigmine contracts the pupil and the ciliary muscle, producing a marked myosis and reducing intraocular pressure. Its myotic effects are noticeable in the horse thirty minutes after administration; in carnivora, in from fifteen to twenty minutes. The elimination of physostigmine, while executed to some extent by bile, saliva, and gastric fluids, is accomplished chiefly by the kidneys, the urine of an animal under its action being capable of poisoning another.

Antagonists, for its effect on the heart, respiration, and pupil, are atropine and chloral hydrate.

Therapeutics. - In colic due to intestinal obstruction, etc., constipation, intestinal torpor-physostigmine combined with equal quantity of pilocarpine. In tetanus-to diminish reflex excitability; in chorea, epilepsy, and as antagonists in strychnine and atropine poisoning; in eye diseases, as a myotic, to relieve congestion and inflammation of the eyeball, to reduce intraocular tension, and, alternated with atropine, to reduce adhesions of the iris; also to re- 
lieve pain and to prevent photophobia. Chronic dropsical conditions of articulations and bursæ of tendons of the horse, after removing the fluid by the aspirator-the cavity is injected with a one-per-cent. solution of physostigmine, and ice or refrigerant lotions applied for several days to combat the resulting inflammation (Stottmeister).

Caution.-Preparations of Calabar bean should never be used in pregnant animals, as the drug stimulates the muscular fibre of the uterus, thus sometimes causing abortion or miscarriage.

\section{Aconitum-ACONIte.}

(Syn.-Wolfsbane, Monkshood, Blue Rocket.)

The tuberous root of Aconitum napellus, natural order Ranunculaceæ, a perennial plant found in mountainous regions, and containing about .03 per cent. of an active alkaloid known as aconitine, in combination with aconitic acid. Other varieties of aconite are said to contain sereral other principles, concerning which there is much division of opinion--viz.: napelline, aconine, pseudaconitine, lyctonine, etc. But it is generally conceded that Aconitum ferox, from Nepaul, contains pseudaconitine, which is much more active than aconitine. Also that a Japanese variety contains an alkaloid known as japaconitine, which is said to be even stronger yet than pseudaconitine.

Preparations.-Extractum Aconiti, extract of aconite. Dose: H., gr. i.-v.; D., gr. $\frac{1}{8-\frac{1}{4}}$. 
Extractum Aconiti Fluidum, fluid extract of aconite. Dose: H., ml v.-xx.; D., $\pi \frac{1}{4}-\mathrm{i}$.

Tinctura Aconiti, U.S. P., tincture of aconite (thirty-five per cent.). Dose: H., $\pi l \mathrm{xv} .-\mathrm{xl}$.; D., m ss.-iij.

Tinctura Aconiti, Fleming's, Fleming's tincture of aconite (seventy-nine per cent.). Dose: H., m viij. $-\mathrm{xv} . ; \mathrm{D} ., \pi \frac{1}{8}-\frac{3}{4}$. Now almost obsolete and not so reliable as the fluid extract.

Aconitina, aconitine. White, flat crystals, of a feebly bitter taste; soluble in alcohol, ether, and chloroform; incompatible with alkalies, tannin, and mercurial salts. Difficult to obtain of a constant and reliable strength. The most reliable are Squibb's nitrate of aconitine and aconitinum purum, Merck. Used hypodermically on the horse in doses of gr. $\frac{1}{30}-\frac{1}{20}$.

Oleatum Aconitince, oleate of aconite (two per cent.). For local use only.

Physiological Action.-Aconite and its preparations are sensory, cardiac, respiratory, and spinal depressants of great activity and power; also antipyretics, diaphoretics, and diuretics, its antipyretic action being due to its sedative action upon the heart and respiration; hence its use is indicated at the offset of acute inflammatory diseases. Small doses, given upon the tongue, soon cause a sensation of numbness and a persistent tingling of the lips and tongue. Full doses cause a feeling of constriction of the fauces, irritation of the gastro-intestinal mucous membrane, with increased secretion, sometimes with 
a slight diarrhœa. In the horse-movements as of deglutition ; in the dog-nausea and sometimes vomiting. Always more or less salivation, diaphoresis, and diuresis; reduced respiratory power, due to depression of respiratory centre, as well as the reduced circulation; decreased heart rate and force; lowered arterial tension and temperature. Toxic doses produce great muscular weakness, impairment of vision, pupils sometimes dilated, occasionally contracted; shallow, irregular, and labored respiration; a slow, weak pulse; coldness and numbness of body and extremities; clammy sweat; abolishment of reflexes, sensation, and motility. The temperature is lowered from $2^{\circ}$ to $3^{\circ} \mathrm{F}$., and death takes place from paralysis of heart and respiration, with or without convulsions, consciousness being preserved until near the end, when $\mathrm{CO}_{2}$ poisoning sets in. Postmortem examination shows shrunken lungs, containing but little blood; trachea and bronchi containing much frothy mucus; right side of heart greatly distended with blood, while left side is almost empty; ecchymotic spots found in lungs, pleura, and endocardium; digestive organs do not appear seriously affected.

Aconite acts chiefly upon the peripheral ends of the sensory nerves, but paralyzes both motor as well as sensory nerves, the latter being affected first and from the periphery inward, while the motor nerves are affected from the centres outward. While not affecting the brain, it relaxes the cardiac inhibitory apparatus, paralyzes the heart muscle and its contained 
ganglia, the respiratory centres, and the spinal cord in all its functions (motor, sensory, and reflex). Aconite is slowly eliminated but rapidly absorbed, and its action continues for from three to four hours; the elimination being accomplished chiefly by the kidneys, increasing both the solid as well as fluid constituents of the urine. Applied externally, it paralyzes the sensory nerve endings of the part; hence acts as a local anodyne and relieving pain.

Aconite Compared with Digitalis.-Aconite at first stimulates, but soon relaxes inhibition and depresses the cardiac motor ganglia. Digitalis increases inhibition and stimulates the heart muscle. Both drugs finally paralyze the heart-aconite by direct depression, digitalis by over-stimulation. Aconite arrests the heart in diastole, digitalis in systole. Aconite lowers arterial tension, digitalis raises it. Aconite acts quickly, digitalis very slowly. Both slow the heart, but otherwise antagonize each other in their cardiac actions.

Antagonists and Antidotes.-Caffeine, atropine, morphine, ether, ammonia, and amyl nitrite antagonize its action on the heart and respiration. Digitalis counteracts its heart action and the relaxation of cardiac inhibition, but as it acts only slowly is not of much service in aconite poisoning. Evacuate stomach if possible, apply warmth and friction to extremities, stimulants; in small patients-artificial respiration; always recumbent position.

Therapeutics.-Aconite is given in carefully regulated doses in pyrexia; in enteritis, peritonitis, 
laminitis, acute rheumatism, acute inflammations of the respiratory apparatus, and acute throat affections; in simple and eruptive fevers, puerperal and surgical fever, diarrhœa and dysentery from cold or exposure; in colic-combined with purgatives; in constipation of plethoric animals, with hot, dry, feverish skin. Aconite liniments are used externally to relieve pain and irritability, but must not be applied over too large a surface, as there might be absorption enough to poison the animal.

\section{VERATRUM-HELLEBORE.}

Varieties of Veratrum.-Veratrum viride, American hellebore or poke root (natural order Liliaceæ); Veratrum album, European hellebore; and Veratrum sabadilla, or Asagroea officinalis, the Mexican variety. Of these the first only is official in the U. S. P., but an alkaloid or mixture of alkaloids from the seeds of the Mexican variety is also recognized under the name of veratrine.

The alkaloids contained in each plant are as follows: In veratrum viride-jervine, pseudo-jervine, cevadine, rubijervine. In veratrum album-jervine, pseudo-jervine, veratralbine, rubijervine. In veratrum sabadilla-veratrine, cevadine, cevadilline.

Veratroidine is now considered a mixture of rubijervine and a toxic resin.

Preparations.-Extractum Veratri. Viridis Fluidum, fluid extract of American hellebore root. Dose: H., fl. 3 ss.-i.; D., $7 \frac{1}{10}-\frac{1}{2}$.

Tinctura Veratri Viridis, tincture of American 
hellebore root (fifty per cent.). Dose: H., fl. 3 ss.ij.; D., Tl $\frac{1}{6}-\mathrm{i}$.

Veratrum Viride (Pulvis), powdered poke root. Dose: H., 3 ss.-i.; D., gr. $\frac{1}{10}-\frac{1}{2}$.

Veratrina, veratrine. An alkaloid or mixture of alkaloids obtained from the seeds of Asagrœa officinalis, natural order Melanthaceæ. A white or grayish-white, amorphous, rarely crystalline powder, permanent in the air, odorless, of a distinctive acrid taste, leaving a sensation of tingling and numbness upon the tongue, producing constriction of the fauces, and highly irritant to the mucous membranes of the upper air passages. It is very slightly soluble in cold or hot water, imparting to it an acrid taste and a feebly alkaline reaction. It is soluble in 3 parts of alcohol, in 6 parts of ether, in 2 of chloroform, in 96 of glycerin, and in 56 parts of olive oil. Dose: H., gr. $\frac{1}{4}$-ij.; D., gr. $\frac{1}{50}-\frac{1}{10}$; hypodermically, one-half of these doses.

Oleatum Veratrince, oleate of veratrine (two per cent.); Unguentum Veratrince, ointment of veratrine (four per cent.). For lucal use only.

Physiological ACtion.-Veratrum viride is a powerful depressant to the heart and spinal functions, a spinal paralyzant, and closely resembles aconite, from which it differs in being a systemic emetocathartic, in paralyzing the motor system centrally, impairing the reflexes, but in having but slight action upon sensation, and in being practically devoid of diaphoretic and diuretic action. Small doses reduce the force of the pulse, and only affect the fre- 
quency of the pulse when continued for some time. It at times causes considerable depression, muscular weakness, nausea, and vomiting. Large doses make the pulse very small, rapid, and almost imperceptible, and frequently cause gastro-enteritis. Respiration is not nearly so much affected as by aconite. Toxic doses cause a fatal cardiac paralysis, preceded by coldness of surface, impaired vision, constant vomiting, and sometimes partial unconsciousness.

Jervine partly represents the depressant action on the circulation and on the central nervous system, while it does not affect the vagus, and but very slightly the voluntary muscles or motor nerves. It irritates the motor centres in the brain, causing convulsions; it lowers the functions of the cord, of the centres in the medulla, especially the vasomotor, and those of the cardiac ganglia, slowing the heart by direct depression either of the cardiac muscle or its motor apparatus. It always causes salivation, but never vomiting or purging; it kills by asphyxia, the heart beating after respiration has ceased.

Veratroidine always causes emeto-catharsis, produces less violent convulsions, stimulates the vagus centre, and paralyzes the vagus ends; depresses the cord and paralyzes the respiratory centre, but at the same time increases the excitability of the vasomotor centre. The result is a great slowing of the pulse rate and of respiration, lowering of the blood pressure, until the carbonized blood irritates the vasomotor centre, when blood pressure greatly rises.

Veratrum album contains a very powerful alkaloid, 
veratralbine, and also an irritant poisonous resin. In action it greatly resembles its congener, but is much more irritant to the gastro-intestinal mucous membrane, causes violent vomiting and purging, intense abdominal and œsophageal pain, greatly reduced temperature and pulse, collapse, and death from paralysis of heart and respiration.

Veratrine causes free salivation, affects the heart and circulation similarly to the other veratriæ, besides being a direct poison to muscular tissue. The alkaloids jervine and rubijervine both depress the circulation, while the latter produces the emeto-catharsis.

Veratrum poisoning should be treated in the same manner as aconite poisoning.

Therapeutics. - Veratrum, although inferior to aconite in fevers and inflammations, because of its lack of power over excretions, is an excellent sedative in the treatment of aneurism, simple hypertrophy, and irritable heart; in puerperal convulsions and fevers; in the earlier stages of parenchymatous and serous inflammations, particularly in pneumonia and enteritis; in rheumatism, laminitis; hypodermic injections of veratrine in the affected muscles in rheumatism and muscular atony; also externally to relieve neuralgic pains, and as an insecticide.

Flores Arnic

The flowering heads of Arnica Montana, natural order Compositæ, a perennial plant of the mountainous regions of the northern hemisphere. It is sometimes known as leopard's bane, and contains a gluco- 
side-like principle-arnicin-which is insoluble in water; also about one per cent. of an essential oil, with inulin, tannin, mucilage, resins, capronic and caprylic acids, and trimethylamine, which is thought to be the active principle, and which is yielded by the essential oil.

Tinctura Arnica Florum (twenty per cent.), tincture of arnica flowers, is the chief preparation. Dose: H., fl. 3 ss.-i.; D., Mlv.-x. This preparation is used mostly externally, more or less diluted. The bruised and moistened flowers are also sometimes used as a poultice. Internally its action is said to be uncertain, but it has been used with good results in pulmonary congestion and lymphangitis by Professor Williams and others, who claim that it quickens the cutaneous circulation. It is an irritant, stimulant, depressant, antipyretic, diuretic, and vulnerary. Small doses increase the heart's action and stimulate the action of the skin and kidneys. Large doses depress the heart and respiration, and reduce temperature. Toxic doses paralyze the entire nervous system, causing collapse and death. Internally-it is employed in rheumatism, lymphangitis, inflammations of serous membranes, in chronic dysentery, typhoid fevers, paralysis of bladder, chorea, concussion of brain, etc. Externally-to bruises, contusions, sprains, wounds, cuts, harness and saddle galls.

\section{The Nitrites.}

Amyl Nitris, amyl nitrite- $\mathrm{C}_{5} \mathrm{H}_{11} \mathrm{NO}_{2}$-is a clear, yellowish, oily liquid, of a powerful and ethereal 
odor, extremely volatile, insoluble in water, but soluble in alcohol, ether, and chloroform. It is obtained by the reaction of nitrous or nitric acid upon amylic alcohol. Dose: H., fl. 3 ss.-ij.; D., Mi.-v.

Glonoinum, glonoin, glyceryl trinitrate, trinitrin, nitroglycerin $-\mathrm{C}_{3} \mathrm{H}_{5}\left(\mathrm{NO}_{3}\right)_{3}$-is a clear, oily liquid, insoluble in water, but soluble in alcohol, and is produced by the reaction of $\mathrm{HNO}_{3}$ and $\mathrm{H}_{2} \mathrm{SO}_{4}$ upon glycerin. It is a very explosive compound, and is therefore usually kept only in one-per-cent. solution in alcohol, which is known as-

Tinctura Trinitrini, one per cent.; Spiritus Glonoini, one per cent. Dose: H., fl. 3 ss.-ij.; D., mlss. $-\mathrm{x}$.

Sodii Nitris, sodium nitrite; Potassce Nitris, potassium nitrite. Dose: H., gr. xv. -3 iss.; D., gr. ss.-iij.

Ethyli Nitris, ethyl nitrite- $\mathrm{C}_{2} \mathrm{H}_{5} \mathrm{NO}_{2}$ - constitutes about five per cent. of spiritus ætheris nitrosi. (See under Ather).

Physiological Action.-The nitrites are said to produce great vascular dilatation, and consequently lower blood pressure. They are all muscle poisons in large doses. They create a tumultuous action of the heart by relaxing its inhibitory apparatus; they diminish sensation, motion, and reflexes; they lower body temperature and respiration.

The amyl nitrite is the most prompt but less enduring in action, and is best given by inhalation. The nitroglycerin is less prompt, less violent, but more lasting in action, and most suitable for internal use. 
Therapeutics. - The nitrites are employed in angina pectoris, asthma, epilepsy, spasmodic cough, vomiting and nausea, puerperal convulsions, chronic interstitial nephritis, tetanus.

\section{ANTIZYMOTICS, ANTIPYRETICS, ANTI- PERIODICS.}

The cinchona alkaloids are the most powerful of these, and have already been described.

\section{ANTIPYRINUM-ANTIPYRIN.}

(Syn.-Phenazone, Methozine, Analgesine, Dimethylphenylpyrazolon.)

A synthetical base, which forms salts analogous to the ammonia compounds, and is a product of the destructive distillation of coal tar. It may also be prepared synthetically. It occurs as a whitish, crystalline, odorless powder, which combines with acids to form salts; slightly bitter taste, soluble in its own weight of water, in alcohol, and chloroform, but not so readily soluble in ether. It is best given alone, as it is incompatible with many drugs, the more important of which are tannic and prussic acids, chloral hydrate, preparations of cinchona bark, ferric salts in solution, infusions and tinctures containing tannin and quinine, tincture of iodine, and corrosive sublimate.

In action it is a powerful antipyretic, antirheumatic, antineuralgic, antiseptic, and hæmostatic. Large doses are apt to irritate the gastro-intestinal mucous membranes, and in the dog to cause vomit- 
ing. This may be avoided by giving the drug in aërated water. It is rapidly absorbed and but slowly eliminated, which last process is carried on chiefly by the kidneys. It does not lower the body temperature in health, but has great power to do so in abnormal body temperature, whether given internally, subcutaneously, or intratracheally. Its effects as a febrifuge are due to diminished heat-production. Occasionally it will produce a profuse perspiration, vertigo, collapse, and in the dog at times a rash resembling that of measles. Antipyrine may be given in all febrile and inflammatory diseases, influenza, acute muscular and articular rheumatism, and diabetes insipidus, in doses for the horse of 3 i.-iv.; for the dog, gr. v. $-\mathrm{xx}$. Externally-it is employed in five- to ten-per-cent. solutions as a hæmostatic; subcutaneous injections of the drug are used to relieve pain and nervous irritability in the treatment of colic (dose about one-third of that per os).

\section{ACETANILIDUM-ACETANILID.}

(Known also as Phenyl Acetamide, Antifebrin [a trade name].)

Acetanilid is obtained by the reaction of glacial acetic acid on anilin. It occurs as colorless or whitish, shining, odorless crystals, having a slightly burning taste and a neutral reaction. Soluble in 190 parts of cold water, in 18 parts of boiling water, and in $3 \frac{1}{2}$ parts of alcohol; also soluble in chloroform and ether. Dose: H., 3 i.-ij.; D., gr. v. $-x v$. 
Physiological Action.-Acetanilid is a powerful antipyretic, analgesic, and antispasmodic, antirheumatic, antineuralgic, diaphoretic, and slight antiseptic. Its action somewhat resembles that of antipyrine, but while this is manifested more slowly it continues for a much longer period-i.e., about six hours. It is a cerebral, muscular, and vasomotor stimulant, and leaves no ill after-effects, such as sometimes follow the administration of antipyrine.

Therapeutics. - It is used in the same diseases in which antipyrine is administered, and also externally as an antiseptic to wounds slow in healing.

\section{Carbolic Acid-Phenol.}

Carbolic acid- $\mathrm{C}_{6} \mathrm{H}_{5} \mathrm{HO}$ or $\mathrm{C}_{6} \mathrm{H}_{6} \mathrm{O}$-also known as phenyl hydrate, phenyl alcohol, phenic acid, and phenol, was discovered in 1848 by Runge, and is obtained from coal tar by fractional distillation and subsequently purified. It occurs as colorless, interlaced, needle-shaped crystals, sometimes acquiring a pinkish tint, deliquescent on exposure, having a distinctive, slightly aromatic odor, resembling creosote; when diluted a sweetish taste, with a slightly burning after-taste and a neutral reaction. It produces a benumbing, blanching, and caustic effect upon the skin, coagulates albumen (difference from Creosote, U. S. P.), is soluble in 20 parts of water, very soluble in alcohol, ether, chloroform, glycerin, fixed and volatile oils.

Acidum Carbolicum Purum Liquefactum, pure 
carbolic acid liquefied. Is given internally in doses of: H., Mx.-xl.; D., Mlss.-ij. Always well diluted in glycerin or oil, or in bolus.

Acidum Carbolicum Crudum, crude carbolic acid. Consists of various constituents of coal tar, chiefly cresol and phenol, and is also obtained by fractional distillation. Used only as a disinfectant and deodorant.

Preparations.-Unguentum Acidi Carbolici (ten per cent.), ointment of carbolic acid. Used locally.

Glycerinum Acidi Carbolici, glycerine of carbolic acid, 1 to 4 of glycerin.

Liquor Acidi Carbolici, solution of carbolic acid. For local use in antiseptic surgery, of various proportions in water; two and a half per cent. for sponges, hands, etc. ; five per cent. as a spray.

Oleum Carbolizatum, carbolized oil. Used locally, contains of carbolic acid 1 part and cotton-seed oil 7 parts.

Derivatives.-Sodii Sulphocarbolas, sodium sulpho-carbolate, prepared by dissolving carbolic acid in an equal part of sulphuric acid, to form sulphocarbolic acid, and neutralizing with barium and sodium carbonates. Occurs in rhombic prisms, soluble in 5 parts of water. Dose: H., 3 ij.-vi.; D., gr. v. $-\mathrm{xx}$. Used internally as an antiseptic and antizymotic, but clinical observation has failed to substantiate the high claims made for it some years ago.

Salol, phenyl salicylate, the salicylic ether of phenyl (see under Salicylic Acid), combines the ac- 
tions of the drugs from which it is derived, and is at once the best and safest manner of administering carbolic acid internally.

Campho-Phenique, a saturated solution of camphor in liquefied carbolic acid, is a most excellent antiseptic, especially adapted to local use. It is markedly anæsthetic and anodyne, and can be applied pure or diluted with oil or glycerin to the most painful and sensitive wound or burn.

Physiological Action.-Carbolic acid is the best known and most commonly used disinfectant and antiseptic, although not the most active. It is an antiferment, a deodorizer, and prevents the decomposition of albuminous fluids by bacteria. Applied locally it is a styptic, anæsthetic, and superficially escharotic, coagulating the albumin of the part; it is powerfully poisonous to the tissues, and when applied directly to muscle or nerve paralyzes these at once without previous stimulation. When given undiluted and in large doses carbolic acid causes violent gastro-enteritis with vomiting and purging, followed by collapse, delirium, and often convulsions and death. After absorption it acts by selection upon the medulla, especially upon the respiratory and vasomotor centres therein, which it first briefly stimulates and then completely paralyzes. It stimulates cardiac inhibition, first slowing the heart, then depressing, and finally paralyzing it. Respiration, at first increased, is soon depressed, the pupils become contracted, the brain and spinal cord are directly affected; stupor, coma, suspended reflexes, impaired motility and sen- 
sibility are produced. It is rapidly absorbed and also rapidly diffused, and fatal cases have occurred from its local use in full strength. The dog and cat are especially sensitive to carbolic acid; hence it must be very carefully used on these animals. It is partly oxidized in the body and partly eliminated by the lungs, skin, and kidneys, imparting to the urine an olive-brown color, and giving this excretion the power of resisting putrefaction for a considerable time. Death from a medium dose occurs by paralysis of respiration; from a large dose by paralysis of the heart. The blood after death is very dark in color and almost non-coagulable.

Antidotes and Antagonists.-Magnesium or sodium sulphate in large doses at once to form a sulphocarbolate; aqua calcis in copious doses, but is not so efficient as the sulphates; atropine is a complete physiological antagonist, maintaining the heart and respiration. Vegetable demulcents-as oatmeal gruel, linseed tea, etc. (but no oils or glycerin) - to protect the mucous surfaces.

ThERAPEUTICS. - As an antiseptic carbolic acid may be applied in surgery and in the treatment of wounds of all kinds, excepting those of the eye. As a wash in footrot among sheep, fistulæ, burns, scalds, injuries of vagina or uterus, resulting from parturition, leucorrhoal and other discharges. Internally -in Texas fever, anthrax, foot-and-mouth diseases catarrhal influenza of horses, strangles, typhoid complaints. As a mouth-wash in stomatitis and glossitis; as an intratracheal injection in calves suffering 
from stranguli (usually combined with oil of turpentine). Externally - as an ointment in grease, scratches, eczema, scab, mange, and to destroy lice and fleas. The crude acid is used as a disinfectant and deodorant in stables, kennels, etc.

\section{Acidum Salicylicum-Salicylic Acid.}

Acidum Salicylicum, Salicylic Acid, an organic acid, which was originally prepared from salicin, a crystalline glucoside obtained from several species of salix (willow) and populus (poplar). Salicylic acid is now prepared synthetically from carbolic acid by the reaction of sodium and carbonic anhydride. It occurs in fine, white, light, prismatic, needle-shaped crystals, permanent in the air, free from odor of carbolic acid, but sometimes having a slight, aromatic odor, of a sweetish and slightly acrid taste and an acid reaction. Soluble in 450 parts of water and in 2.5 parts of alcohol, in 2 parts of ether, and in 80 parts of chloroform. Dose: H., 3 ij.-vi.; D., gr. v.-xv.

Preparations.-Sodii Salicylas, sodium salicylate. Small, white, crystalline plates, or a crystalline powder, permanent in the air, odorless, having a sweetish, saline, and mildly alkaline taste and a feebly acid reaction. Soluble in 1.5 parts of water and in 6 parts of alcohol.

Lithii Salicylas, lithium salicylate. A white powder, deliquescent on exposure to air, odorless or nearly so, having a sweetish taste and a faintly acid reaction. Very soluble in water and in alcohol.

Salol, salicylate of phenyl. A fine, crystalline, 
white powder, having an aromatic odor and insipid taste. Soluble in 10 parts of alcohol; also in chloroform, ether, and fixed oils; almost insoluble in water. When heated with an alkali, salol splits up into salicylic acid 60 parts, and carbolic acid 40 parts.

The doses of these three preparations of salicylic acid are: H., 3 iv. -3 i.; D., gr. v. -3 ss.

Physiólogical ACtion.-Salicylic acid is an antipyretic, antiseptic, antiperiodic, antirheumatic, and antiferment. Small doses stimulate the stomach, heart, and respiration. Large doses cause gastric derangement, nausea, vomiting, and sometimes diarrhœa in all animals; depress the heart and respiration, lower arterial tension, and reduce temperature in fever. Continued large doses cause symptoms resembling cinchonism (see Quinine). Toxic doses produce death by paralysis of respiration. It does not stimulate the flow of bile, but renders this secretion more watery, and is excreted by the skin, salivary glands, and kidneys, the latter organs being sometimes so irritated by the drug as to cause albuminuria. Sodium salicylate, although much resembling salicylic acid in action, is devoid of the antiseptic properties of the latter. It is also a diaphoretic and cholagogue, being supposed to possess the property of increasing the fluidity of the bile, at the same time promoting the secretion of the latter, while other cholagogues increase the proportion of solids in the bile. The corresponding lithium salt closely resembles this, but has more power over excretion.

Salol is antiseptic, analgesic, a good antipyretic, 
diaphoretic, and antiferment, of more potency than either of its constituents; is not so toxic, nor so irritant and depressant. It is also sedative to the cerebro-spinal system.

Therapeutics.-Salicylic acid is employed in acute catarrhal conditions of the respiratory apparatus, in influenza, rheumatism, purpura, peritonitis, in fevers, especially of the septicæmic kind; as an antiferment in colic, tympanites, etc., in the horse, cattle, and sheep. Externally-in eczema, parasitic skin affections, to gangrenous wounds, etc.

Sodium and lithium salicylate-for the same purposes as the acid, but are preferable in rheumatic disorders.

Salol, besides the uses already mentioned, is one of the best disinfectants for the gut, being an efficient remedy for duodenal catarrh, vesical catarrh, etc., - and as an antiseptic for the urinary apparatus. As a febrifuge it supersedes salicylic acid and its other preparations. It is much employed in infectious diarrhœa in calves, sheep, and dogs.

\section{Thymol and Menthol.}

Thymol- $\mathrm{C}_{10} \mathrm{H}_{14} \mathrm{O}$-is a phenol contained in oil of thyme and in the volatile oils of several other plants. It occurs as large hexagonal crystals, nearly or quite colorless, having an aromatic, thyme-like odor, a pungent, aromatic taste, with a very slight caustic effect upon the lips, and a neutral reaction. Soluble in 1,200 parts of water and in 1 part of alcohol; also in ether, chloroform, glacial acetic acid, fixed and 
volatile oils. It liquefies when triturated with equal quantities of camphor, menthol, or chloral.

Thymol resembles both carbolic acid and oil of turpentine in its action, being a powerful antiseptic and germicide like the former, and like the latter an irritant to the organs of elimination and a paralyzant. Toxic doses, when absorbed, paralyze the nerve centres in the spinal cord and medulla from the first, with no preliminary stimulation-slowing respiration, lowering arterial tension and body-temperature, as well as lessening reflex excitability. It is eliminated by the kidneys and respiratory organs, causing more or less irritation at the points of elimination. Autopsies on animals poisoned by it show fatty degeneration of the liver (as with phosphorus), and great congestion of the bronchial and pulmonary mucous membranes and of the kidneys.

Thymol is employed as an antiseptic application to wounds and skin diseases, and as a spray or inhalation in chronic affections of the lungs and bronchi. It has considerable repute in vesical catarrh. Dose: H., gr. X.-xxx. ; D., gr. ss.-v.

Menthol, peppermint camphor- $\mathrm{C}_{10} \mathrm{H}_{19} \mathrm{OH}$-is a stearopten, isomeric with thymol, and is obtained from oil of peppermint by deposit on exposure to cold. It occurs in colorless crystals or masses, having the characteristic taste and odor of peppermint oil. Soluble in alcohol, ether, and chloroform, but only sparingly soluble in water. Dose: H., gr.xv.-3 ss.; D., gr. ss.-iij.

Oleum Menthce Piperitoe, oil of peppermint, is the 
volatile oil obtained by distillation from the fresh herb, and contains menthol and a liquid terpene. Dose: H., fl. 3 ss.-i. ; D., Tliij.-v.

Aqua Menthoe Piperitce, peppermint water, containing one drop of the oil to the fluidounce of water. Used as a vehicle, chiefly in canine practice.

Spiritus Menthoe Piperito, essence of peppermint (containing ten per cent. of the oil and one per cent. of the powdered herb). Dose: H., fl. 3 ss.-i.; D., mxv. $-3 \mathrm{i}$.

Menthol is a local vascular stimulant when applied to the skin, a local anæsthetic and parasticide, and is non-corrosive. Internally - carminative, artispasmodic, and somewhat antifermentative. It stimulates the secretory nerves and cardiac muscle, and like camphor causes a periodic increase in arterial tension, but does not affect the pulse rate. It inereases the number of the respirations but lessens their depth. It lessens sensation and reflex sensibility, in large doses destroying both, and paralyzing the cerebro-spinal system. Menthol and peppermint preparations are used internally as carminatives, antispasmodics, and stimulants, to relieve colic and flatulence; as corrigents to purgatives to prevent griping. Menthol combined with ether and chloroform is much used as a local anæsthetic.

\section{Chlorum-Chlorine.}

Chlorine- $\mathrm{Cl}$-is a greenish-yellow gas, belonging to the halogen group of elements. Some of the chlorine compounds have been already described under 
Ammonium, Potassium, Sodium, Hydrochloric Acid, etc.

Chlorine is the most powerful of all the disinfectants and deodorants, an antiseptic and antiferment of great activity, its power being due to its affinity for $\mathrm{H}$, decomposing all bodies which contain this element as a molecular constituent, forming $\mathrm{HCl}$, and setting $\mathrm{O}$ free in its nascent form of ozone. Locally chlorine is highly irritant to the skin and mucous membranes. When inhaled in quantity it causes cough, sneezing, spasm of the glottis, and inflammation of the respiratory mucous membranes and of the lungs. For actions and uses of its various compounds, see under their respective headings.

\section{EVACUENTS.}

This division of materia medica includes all remedies which promote some evacuation from the body -as the emetics, cathartics, anthelmintics, diuretics, etc.

\section{EMETICS.}

Cupri Sulphas, copper sulphate. See under Copper.

Zinci Sulphas, zinc sulphate. See under Zinc.

Hydrargyri Subsulphas Flavus, yellow mercuric subsulphate, turpeth mineral. For the dog, gr. iij.v., with sugar of milk. One of the best and very prompt. 
Alumen, alum. See under Alum. Safe, but slow in action.

Sinapis Alba, white or yellow mustard; Sinapis Nigra, black mustard. See under Sinapis (topical agents).

Apomorphina, apomorphine. See under Opium. Very depressant and convulsant.

Antimonii et Potassce Tartras, Tartarus Stibiatus, tartrate of antimony and potassium, tartar emetic. Given to the dog in doses of gr. i.-iv. dissolved in tepid water.

Emetics are given to cause expulsion of foreign bodies from any part of the œsophageal tract oi from the air passages, to remove or evacuate the contents of the stomach, and to remove an obstruction from the gall duct. Contra-indications for their use areaneurism, atheroma, hemorrhagic diathesis, hernia, prolapsus uteri, and pregnancy.

\section{IPECACUANHA-IPECAC.}

The dried root of Cephaëlis ipecacuanha, a Brazilian shrub of the natural order Rubiaceæ, collected during the first three months of the year. The shrub grows about three feet high; the root has a twisted knotty appearance, of about the thickness of a quill, and is from two to four inches long. The active principle is contained in the outside brownish bark, while the inner part of the root is white and nearly inactive, medicinally. The powdered root is of a grayish-brown color, of a bitter, acrid taste, and has 
a faint nauseating odor. The root contains a crystalline alkaloid, emetine; a glucoside, ipecacuanhic acid; also gum, starch, etc., and a trace of a volatile oil.

Preparations.-Pulvis Ipecacuanhoe, powdered ipecac root. Dose: H., 3 ss.-ij.; D., gr. ss.-ij.; as an emetic, gr. $\mathrm{x} .-\mathrm{xxx}$.

Extractum Ipecacuanhoe Fluidum, fluid extract of ipecac. Dose: H., fl. 3 ss.-ij.; D., Mss.-ij.; as emetic, $\Pi \times x v .-x x x$.

Syrupus Ipecacuanho, syrup of ipecac (seven per cent.). Dose: D., fl. 3 i.-iv.

Pulvis Ipecacuanhoe et Opii, powder of ipecac and opium, Dover's powder. Contains ipecac 10, opium 10, sugar of milk 80. Dose: H., 3 i.-iij. ; D., gr. v. $-\mathrm{xv}$.

Physiological Action and Uses.-Ipecac is a cholagogue, diaphoretic, expectorant, and emetic for dogs, cats, and pigs. Full doses, sufficient to cause emesis, when given to animals which do not vomit, produce an increased amount of bronchial secretion; hence are given in catarrh and bronchitis when cough is "hard and fast." The powder when inhaled causes sneezing, and is sometimes used as a sternutatory. Medicinal doses increase all the mucous secretions, the hepatic secretions, and the sweat glands. Continued use begets tolerance. Small doses act as a stomachic tonic and stimulant to the gastric secretions. As a diaphoretic the Dover's powder is much used in rheumatism, diarrhœa, and dysentery, in the latter troubles being given in large doses up to tolerance. As an expectorant in bronchitis, spasmodic 
cough, and other affections of the respiratory apparatus. In hepatic congestion, jaundice, etc., with calomel and aloes. As an emetic to relieve the stomach in acute indigestion of the dog; in small doses to relieve vomiting due to nervous excitement.

\section{CATHARTICS.}

Cathartics are divided into laxatives, and simple, saline, drastic, hydragogue, and cholagogue purgatives.

\section{LAXATIVES.}

Sulphur Lotum, Sulphur Procipitatum. See under Sulphur.

Magnesii Carbonas. See under Magnesium.

Oleum Ricini, castor oil, the fixed oil expressed from the seeds of Ricinus communis. The seeds contain about fifty per cent. of the oil, which is obtained by expression, without the aid of heat. The oil is purified by rest, is sometimes filtered, and even bleached. It is a mild laxative; becomes partly emulsified, and this part of it is absorbed; but the greater part passes on through the intestines, stimulating secretion and peristalsis without griping, and frequently may be detected in the frces. It is given in diarrhœa and dysentery, and constipation during pregnancy, etc. Dose: H., 亏 fl.xij.-xvi.; D., fl. 亏 ss. -ij.

Fruits, roots, green food, bran mashes, etc., also act as laxatives. 


\section{Simple Purgatives.}

Rheum, rhubarb (Radix rhei), the dried and sliced root of Rheum palmatum and other rhubarb species. Obtained chiefly from China and Thibet. It is stomachic, tonic, astringent, a mild cathartic and cholagogue; increases the secretion of bile. Small repeated doses improve digestion and assist to correct slight gastric derangement; large doses are mild cathartics in the dog and cat. Dose as stomachic and tonic for the dog, gr. x.-xx.; as cathartic, 3 i.-iij.

Aloë, aloes, the inspissated juice of the leaves of Aloë vera (Barbadoes aloes) and other aloes species. Contains aloin, a neutral principle common to all varieties of the plant, of which three are found in the market.

1. Aloë Barbadensis, Barbadoes aloes, obtained from the West Indies, is of a liver-brown color, an opaque, earthy fracture, a persistent, disagreeably bitter taste, a strong, disagreeable odor. When breathed upon, it gives off an odor resembling that of the human axilla. It is tough and hard to pulverize, and its powder is a dull olive yellow and darker than that of the other varieties. It is almost entirely soluble in proof spirit.

2. Aloë Socotrince, socotrine aloes, from Fast India, Bombay, and Zanzibar. Reddish-brown pieces of various sizes, breaking with a smooth, resinous fracture. Darkens on exposure to air; odor, although strong, is somewhat agreeable; taste, very bitter. 
3. Aloë Capiensis, Cape aloes, from Cape Town, Natal, etc. The better qualities are equal almost to the Barbadoes and socotrine aloes, but give less watery discharges, and the action does not continue for so long a time. Olive-green, resinous appearance; strong, disagreeable, sour odor; bitter taste. The pieces are very brittle, are easily powdered, and the powder has the appearance of chrome jellow or gamboge.

Physiological Action.-Aloes is chiefly a pur. gative, stimulant, and bitter tonic; also anthelmintic. Externally-a stimulant and desiccant. When given internally aloes is dissolved by the gastric juice, saponified by the pancreatic juice and bile, enters the circulation, and communicates its bitterness and purgative qualities to milk and other secretions. It is excreted by the intestinal glandular apparatus and kidneys. Large doses cause hyperæmia of the uterus, kidneys, and other pelvic organs. It increases peristalsis, also the amount of secretion in the intestines; but its action is confined chiefly to the large gut. The secretion of bile is also stimulated. It requires from twelve to twenty-four hours to operate. It is the most suitable purgative for the equine species, and should be given in bolus or drench, the latter acting somewhat more rapidly. When given to cattle it is usually more active when conjoined with salines.

Therapeutics. - Aloes is given in small doses as a bitter tonic in dyspepsia, irregularity of the bowel; as a purgative in constipation; in torpidity of the 
bowel and habitual constipation-combined with nux vomica; in enfeebled and relaxed conditions of the alimentary canal-combined with iron and in small doses; in spasmodic and flatulent colic-with anodynes and carminatives; in acute indigestion, to carry off the contents of the stomach and intestines; in azoturia in very large doses, usually as a drench, with other depletives; in lymphangitis - combined with digitalis; as an anthelmintic-combined with santonin, ether, oil of male fern, oil of turpentine, etc.; in torpidity of the liver-with calomel. As a purgative for the horse, in order to insure a free action, the animal should be first prepared with bran mashes for two meals; an hour or two after the last mash the ball should be given, and ten to twelve hours later the animal should be walked about for a short time. A very good and efficient bolus is composed of Aloes 3 vi., fluid extract belladonna $3 \mathrm{i}$, powdered capsicum 3 i., powdered nux vomica $3 i$. The ingredients may be increased in dose to suit the requirements of any particular case. Aloes should not be given if the alimentary canal is seriously inflamed or irritated; nor in severe respiratory diseases, pregnancy, hernia, rectal hemorrhage, or prolapsus ani. A purgative dose of aloes should not be repeated until at least thirty-six hours after the first has been given, as superpurgation is readily induced. Dose: as a purgative, H., 3 ss.-i.; C., $\xi$ i.-iij.; D., gr. xx.- $3 \mathrm{i}$.; as a tonic, one-sixth to one-eighth of these doses suffice.

Aloes has been used externally as tinctura aloës et 
myrrhæ, a stimulant to wounds, etc. The powder is mixed with plaster of Paris when applying the latter to fractured limbs of dogs, etc., to prevent these animals from tearing off their bandages.

Oleum Lini Seminis, linseed or flaxseed oil, the fixed oil expressed from the seeds of Linum usitatissimum, without heat (see also under oils and fats). A yellowish, oily liquid, having a slight, peculiar odor, a bland taste, and a neutral reaction. When exposed to air it gradually thickens, acquires a strong odor and taste, and finally solidifies. Specific gravity about 0.936 . It is soluble in 5 parts of absolute alcohol and in 1.5 parts of ether. The oil is given as a cathartic to all animals, and as it acts without griping is especially useful in irritable and inflamed conditions of the alimentary canal and urinary apparatus, and when the use of other and more active purgatives is excluded. For cattle it is combined w1th oleum tiglii to increase its activity. Small doses are given with mashes as a food to animals recovering from exhausting diseases. Laxative enemata are composed of the oil with a small quantity of turpentine. Dose: H., O ss.-i.; D., fl. 3 i.-ij.

Oleum Ricini, castor oil. In large doses is quite active, particularly in the dog.

The Buckthorns include: Buckthorn berries--of the Rhamnus catharticus or purging buckthorn; buckthorn bark-of the Rhamnus frangula or black alder; and the dried bark of the Rhamnus purshiana, Cascara sagrada (chittem or sacred bark), sometimes known as California buckthorn. 
They are all cathartics for the dog and cat, but are not so active in the larger animals. The cascara sagrada is particularly useful in the habitual constipation of house-raised and pampered animals. The fluid extract of the first two is frequently combined with ammonia muriate and given to horses suffering frum congested and inactive liver, after having first given an aloes purge. Dose of the fluid extract of any of the Buckthorns: H., fl. 3 ss.-ij.; D., 3 ss.-ij.

\section{Saline Purgatives.}

Magnesii Carbonas, carbonate of magnesium. Light, white friable masses, or a light, white powder, odorless and tasteless. Insoluble in alcohol, and almost insoluble in water, to which it imparts a feebly alkaline reaction; soluble in dilute $\mathrm{HCl}$ with copious effervescence. Dose: D., gr. v.-xxx.

Pulvis Magnesii cum Rheo, Pulvis Rhei Compositus, rhubarb and magnesia, or compound powder of rhubarb. Contains rhubarb 25 , magnesia 65 , and ginger 10. Dose: D., gr. x. $-\mathrm{xxx}$.

Magnesii Sulphas, sulphate of magnesia, Epsom salt. Found in various rocks, soil, in sea-water, and some mineral springs (it is named after the mineral springs of Epsom). Small, colorless, right-rhombic prisms, or acicular needles, slowly efflorescent in dry air, odorless, having a cooling, saline, and bitter taste and a neutral reaction. Soluble in 0.8 part of water, insoluble in alcohol. Somewhat resembles sulphate of zinc and also oxalic acid. It is distinguished 
from the zinc compound by its taste, absence of metallic astringency, and by its neutral solution, giving no precipitate with hydrogen sulphide; distinguished from oxalic acid by its finer and more needle-shaped crystals, its bitter taste, its lack of acid reaction, and its property of precipitating alkaline carbonates without effervescence. Dose: cathartic, H., lb. ss.-i.; C., lb. i.-iss.; D., 3 ij.-iv. As a febrifuge and alterative, one-eighth to one-fifth of these doses suffice. Its activity as a purgative may be somewhat increased by the addition of diluted sulphuric acid.

The magnesia salts are non-poisonous; when given internally they are slowly absorbed. The carbonate is used as a laxative and antacid. Its laxative properties are somewhat increased when the contents of the intestine and stomach are acid; hence it is given to calves and foals suffering from acidity and flatulence; also to dogs and cats. The sulphate is purgative, alterative, febrifuge, and also slightly diaphoretic and diuretic, being excreted by the kidneys and skin. For ruminants it is a good purgative, but on the horse its action is rather uncertain. It is administered in indigestion, constipation, etc., and is usually given with some carminatives; as a febrifuge to horses affected with influenza, pneumonia, and other febrile disorders, when there is any tendency to constipation; also as an antidote in lead-poisoning.

Potassii Bitartras, potassium bitartrate, cream of tartar. Colorless or slightly opaque, rhombic crystals, or a white, somewhat gritty powder, permanent in the air, odorless, having a pleasant, acidulous 
taste and an acid reaction. Soluble in 210 parts of water, slightly soluble in alcohol. In action somewhat resembles the acetate and nitrate of potassium. In small doses it is diuretic, and larger doses purgative. Dose: H., 亏 iv.-viij.; D., 3 i.-iv.

Sodii Sulphas, sulphate of sodium, Glauber's salt. See under Sodium.

\section{Drastic Purgatives.}

Jalapa, jalap, the tuberous root of Ipomca jalapa, a Mexican plant, of the natural order Convolvulaceæ. Contains fifteen to twenty per cent. of the official resin, which is composed of two resins-jalapin and convolvulin - the latter being the more active of the two. It is a hydragogue cathartic, a vermifuge, and cholagogue. It is neither so powerful nor so irritant as gamboge, colocynth, or elaterium. Its cathartic action upon horses and cattle is very slight, two ounces having been given to the horse with but a gentle cathartic effect, but marked diuresis. For dogs and pigs it is a good purgative, although at times causing nausea and vomiting. Dose: D., 3 ss.-ij., of the powdered root.

Oleum Tiglii, oleum crotonis, croton oil. A fixed oil obtained by expression from the seeds of Croton tiglium, an East Indian tree. The seeds contain about fifty per cent. of the oil, which is of a brownish-yellow color, somewhat viscid, and slightly fluorescent; a slight, fatty odor, a mild, oily, afterward acrid and burning taste, and a slightly acid reaction. 
Specific gravity, 0.940 to 0.955 . When fresh it is soluble in about 60 parts of alcohol, the solubility and therapeutic activity increasing with age. It is freely soluble in ether, chloroform, and other oils. Contains several fatty and volatile acids, one of which is known as tiglinic acid. Dose: H., $\Pi \times x v .-x x x$; C., fl. 3 ss.i.; D., mi.-iij.

Croton oil is a drastic purgative, producing copious watery evacuations. It is somewhat too irritant for general use on the horse, and is more often employed in cattle practice, since it acts quicker than aloes. Large doses act as a corrosive poison. Externally it is a powerful irritant, causing a special vesicular eruption, which gradually becomes pustular. On account of its liability of leaving a permanent blemish, it is but little used externally. On the dog and pig its purgative qualities are not accompanied by as much irritation as with the horse.

Gambogia, gamboge, gum gutti, a gum resin obtained from Garcinia hanburii, a medium-sized tropical tree of Siam, Burmah, and Ceylon. It is a very powerful irritant and drastic, hydragogue cathartic, given chiefly to cattle in obstinate constipation, brain diseases, parturient apoplexy, etc., and is second only to croton oil for activity. It is usually conjoined in smaller doses with other cathartics-as Epsom or Glauber's salt. Dose: C., 3 ss.-i.; S., gr. xx.-xxx.

Eserince Salicylas, Chrysostigminoe Salicylas.See under Calabar Bean.

Barii Chloridum, barium chloride- $\left(\mathrm{BaCl}_{2}+\right.$ $\left.2 \mathrm{H}_{2} \mathrm{O}\right)$-colorless, flat, four-sided crystals, of a bitter, 
salty taste. Soluble in $2 \frac{1}{2}$ parts of water, almost insoluble in alcohol. Used as a cardiac tonic and alterative in weakened heart's action. Externally-as collyrium in conjunctivitis and wound dressing; $\frac{1}{2}$ to 2 parts to 100 of water. Professor Dieckerhoff's recent experiments on horses, cattle, and sheep show that it is a powerful and rapid purgative, but which must be cautiously employed since clinical experience and observation of its actions are still incomplete. It is said not seriously to affect either respiration or circulation when given in doses large enough to cause catharsis. The impure chloride of barium has long been used as a "rat poison." Dose: H., 3 ss.-iij., per os; if intravenously, gr. v.-xx., dissolved in distilled water. N.B.: Intravenous injections require great care!

\section{Cholagogue Purgatives.}

Podophyllum, May-apple, the rhizome and rootlets of Podophyllum peltatum or mandrake (natural order Berberideæ). Its active principle is:

Resina Podophylli, resin of podophyllum. Dose: H., 3 i.-ij.; D., gr. ss.-ij.

On horses and cattle its cathartic action is inferior to that of other purgatives, but its cholagogue effects are undoubted; anthelmintic properties are also attributed to it, and in the dog it sometimes causes nausea and vomiting.

Hydrargyri Chloridum Mite, calomel; Hydrargyrum cum creta, gray powder; Massa Hydrargyri, blue mass. See under Hydrargyrum. 


\section{ANTHELMINTICS.}

Santonica, Levant wormseed, the unexpanded flower-heads of Artemisia pauciflora. Contains santonin, a crystalline neutral principle.

Santoninum, santonin. Colorless, shining, flattened, prismatic crystals, permanent in air, but turning yellow on exposure to light; odorless and nearly tasteless when first placed in the mouth, but afterward bitter and having a neutral reaction. Nearly insoluble in cold water, soluble in 40 parts of alcohol, in 160 parts of ether, in 4 parts of chloroform, and in solutions of the alkalies. The alcoholic and ethereal solutions have an intensely bitter taste. It should be kept in dark, amber-colored phials, and protected from light. Dose: H., gr. xv.-xl.; D., gr. ij.-iv. Large doses cause giddiness, vomiting, and convulsions in dogs; medicinal doses are destructive to round- and threadworms, particularly in the dog, the horse being not so much affected. It imparts a blood-red color to the urine.

Arecce Semina, seeds of Areca catechu, areca nut, Betel nut; natural order Palmaceæ. The tree is a native of Asia, and the nut or seed contains an active alkaloid-arecoline.

Pulvis Arecoe Seminis, powdered areca nut, is an active vermifuge, destructive alike to tape- and roundworms, and is most effectual in the dog. Must be given with a cathartic, as it has some astringent properties. Dose: H., 3 iv.-vi.; D., gr. xv. $-3 \mathrm{ij}$. 
(Two grains may be given for each pound of the animal's weight.-Mayhew.)

Chenopodium, American wormseed, the fruit of Chenopodium ambrosiodes.

Oleum Chenopodii, oil of wormseed. A volatile oil, obtained from the American wormseed; destructive to roundworms. Dose: D., Mv. $-\mathrm{xv}$., thrice daily, followed by a cathartic.

Aspidium, filix mas, male fern. The rhizome of Dryopteris filix mas and Dryopteris marginalis. Contains filicic acid, a green, fatty oil, a volatile oil, etc.

Oleoresina Aspidii, oleoresin of male fern, extract of male fern. Dose: H. and C., fl. 3 ij.-viij.; D. and C., Mx.-fl. 3 i.; Sheep and Pigs, fl. 3 i.-ij. The most efficient remedy for tapeworm in all of the dornesticated animals; should be given with a purgative. Large doses are gastro-intestinal irritants.

Oleum Terebinthino, oil of turpentine. See under Turpentine.

The anthelmintics used against the ascarides (roundworms) are: For horses and cattle-aloes, oil of turpentine, bitters, creolin, calx sulphurata, ferric chloride in solution, copper sulphate, arsenic, sulphur, salt in manger; for dogs and cats-santonin, wormseed, oil of male fern, followed by a laxative.

Against the oxyures (threadworms)-enemata of alum, aqua calcis, carbolic acid, vinegar, turpentine, sodium chloride, sulphur, and soft soap.

Against strongyli: In the digestive tract-oil of turpentine, decoction of aloes, and cathartics; in the 
air passages-intratracheal injections of oil of turpentine with carbolic acid and chloroform; sulphurous or chlorine inhalations.

Against tapeworms: For horses and cattle-oil of turpentine and oil of male fern with chloroform; follow with a cathartic. For dogs and cats-areca nut, chloroform, male fern, or calx sulphurata; follow with a cathartic.

\section{DIURETICS.}

\section{Refrigerant Diuretics.}

Potassium Salts, particularly the acetate, bitartrate, carbonate, and nitrate. They should be given largely diluted with water, as that of itself assists to increase diuresis. See under Potassium.

\section{Hydragogue Diuretics.}

Spiritus Atheris Nitrosi, sweet spirit of nitre. See under Ether.

Digitalis, foxglove. See under Digitalis.

Strophanthus and Convallaria. See under their respective headings.

Cocaine. Small doses of the hydrochlorate. See under Cocaine.

Caffeine and Calomel. See under Caffeine and Theine, and under Mercury.

\section{Stimulant Diuretics.}

Buchu Folia, buchu leaves. The leaves of Barosma betelina and other species of Barosma. 
Extractum Buchu Fluidum, fluid extract of buchu. Dose: H., fl. 3 ij.-vi.; D., Tlx.-fl. 3 ss.

Capsicum, Cayenne pepper, the fruit of Capsicum fastigatum. Contains capsicin, a peculiar liquid principle; also a volatile alkaloid.

Oleoresina Capsici, oleoresin of capsicum. Dose: H., Mxv.-fl. 3 i.; D., Mi.--v.

Extractum Capsici Fluidum, fluid extract of capsicum. Dose: H., fl. 3 ij.-vi.; D., Tlv.-fl. 3 ss.

Tinctura Capsici, tincture of capsicum (five per cent.). Dose: H., fl. 3 ss.-iss.; D., Mxv.-fl. 3 i.

Capsicum Pulvis, powdered capsicum. Dose: H., 3 i.-ij.; D., gr. v. $-\mathrm{x}$.

Capsicum in properly regulated doses is stomachic, carminative, sialogogue, and diuretic; large doses are gastro-intestinal irritants. Externally it is used as a stimulant and counter-irritant.

Juniperus, juniper, the fruit of Juniperus communis. Contains juniperin, a non-crystalline principle, and a volatile oil.

Oleum Juniperi, oil of juniper (berries). Dose: H., fl. 3 i.-ij.; D., 卂lv.-xx.

The refrigerant diuretics modify rather than increase the urine, exercising a sedative action upon the heart and circulation. Hydragogue diuretics increase the water of the urine largely, and in general act by raising arterial pressure, either throughout the entire body or locally in the kidneys. The stimulant diuretics are largely eliminated by the kidneys, and act upon the entire genito-urinary mucous membrane by local irritation, which in excess causes in- 
flammation and symptoms of a violent character, as strangury, bloody urine, etc., and therefore should be used with caution. Diuretics are employed to remove fluid from the tissues and cavities of the body in cases of dropsy; to promote the elimination of waste products and other poisons from the blood; to maintain the action of the kidneys; and to dilute the urine and alter morbid conditions of that excretion.

\section{PITCH, TAR, TURPENTINE, ETC.}

The turpentines are liquid or concrete oleoresinous exudations, obtained from various species of the conebearers or Coniferæ, and consist of a resin combined with an oily substance, known as the oil of turpentine.

The three chief turpentines are:

Terebinthina, turpentine, a concrete oleoresin, obtained from Pinus australis or yellow pine, and other species of pinus (natural order Coniferæ), in yellowish, tough masses, brittle in the cold, crummy, crystalline in the interior, of a terebinthinate odor and taste. Dose: as stimulant, antispasmodic, and diuretic, H. and C., ss.-iss. ; D., gr. 3 x.-xxx. ; as anthelmintic, H. and C., 亏 i.-iij.; D., gr. xx.-3i.

Terebinthina Canadensis, Canada turpentine, balsam of fir. A liquid oleoresin, obtained from Abies balsamea, the "silver fir" or "balm of Gilead," natural order Coniferæ. A yellowish or faintly greenish, transparent, viscid liquid, of an agreeable terebinthinate odor, and a bitterish, slightly acrid 
taste; slowly drying on exposure, and then forming a transparent mass; completely soluble in ether, chloroform, or benzol. Dose same as terebinthina.

Terebinthina Veneta, Venice turpentine, from the common larch-Larix Europœa. Is a tenacious, slightly opaque, thick, pale-yellow liquid, of an acrid, bitter taste, and a disagreeable terebinthinate odor. Dose same as terebinthina.

Preparations of Turpentine.-Oleum Terebinthince, oil of turpentine, commonly known as spirits of turpentine, a volatile oil, distilled from the different varieties of turpentine. A thin, colorless fluid, of a characteristic odor and taste, becoming stronger and less pleasant by age and exposure to air, and of a neutral or faintly acid reaction. Specific gravity 0.855 to $0.8 \%$. Soluble in 6 parts of alcohol, miscible with fixed and volatile oils, dissolves resins, wax, sulphur, and phosphorus. Bromine and iodine act violently upon it, and it takes fire when in contact with a mixture of $\mathrm{HNO}_{3}$ and $\mathrm{H}_{2} \mathrm{SO}_{4}$.

Oleum Terebinthince Rectificatum, rectified oil of turpentine, is the preparation of the oil suitable for internal use. Dose: as stimulant and antispasmodic, H. and C., fl. 3 ss. - ij. ; D., $\Pi$ xx. - fl. 3 i.; as diuretic, H. and C., fl. 3 ij.-viij.; D., חlx.-xv.; as cathartic and anthelmintic, H. and C., fl. $\zeta \mathrm{ij}$., with aloes in solution, linseed oil, gentian, or quassia.

Oil of turpentine must be given always diluted with some bland liquid, such as linseed tea or oil, milk, gruel, etc.

Oleum Nigrum, black oil. Consists of oil of tur- 
pentine $\xi \mathrm{iv}$., added to linseed oil $\mathrm{O} \mathrm{i}$; to this add slowly $\mathrm{H}_{2} \mathrm{SO}_{4} z \mathrm{i}$. This mixture must be cautiously prepared, as it evolves great heat.

Derivatives of Turpentine.-Resina, resin, colophony. The residue left after distilling of the volatile oil from turpentine; it is that portion of turpentine which is fixed by oxidation. A transparent, amber-colored substance, hard, brittle, with a glossy and shallow conchoidal fracture, and having a faintly terebinthinate odor and taste. Specific gravity 1.070 to 1.080. It is soluble in alcohol, ether, fixed and volatile oils. Dose same as terebinthina.

Ceratum Resince, unguentum basiliconis, resin cerate, basilicon ointment. Composed of resin 35, yellow wax 15 , lard 50 parts.

Terebeneum, terebene- $\mathrm{C}_{10} \mathrm{H}_{16}$-is a hydrocarbon obtained by the oxidation of oil of turpentine by means of sulphuric acid. A colorless liquid, of a hot taste, soluble in alcohol. Dose, one-half to threefourths of that of oil of turpentine.

Analogues of Turpentine.-Pix, pitch, is a resinous exudation from the stems of certain trees of the genera Pinus (pine) and Abies (fir and spruce). It may also be obtained by the evaporation of woodtar. Three varieties of pitch are in general use:

1. Pix Burgundica, Burgundy pitch, the prepared resinous exudation of Abies excelsa, Norway spruce, natural order Coniferæ. Hard, brittle, opaque masses, very fusible, readily soluble in glacial acetic acid.

2. Pix Canadensis, Canada or hemlock pitch, the 
prepared resinous exudation of Abies Canadensis, the hemlock spruce of the United States and Canada; natural order Coniferæ.

These two varieties are used chiefly in making plasters.

3. Pix Liquida, tar, an empyreumatic oleoresin, obtained by the destructive distillation of the wood of Pinus pallustris and other species of Pinus, natural order Coniferæ. A thick, viscid, semi-fluid, of a brownish-black color, heavier than water, transparent in thin layers, becoming granular and opaque by age; having an acid reaction, an empyreumatic, terebinthinate odor, and a sharp, empyreumatic taste. Slightly soluble in water, soluble in alcohol, fixed or volatile oils, and in solution of potassa or of soda. It consists of pyroligneous acid, methyl alcohol, acetic acid, creosote, toluine, xylene, and other hydrocarbons; also oily bodies, especially the oil of tar and pyrocatechin, a crystalline principle, which gives it its granular appearance. Dose: H., 亏ss.-ij.; D., gr. $\mathrm{x} . \mathrm{xx}$.

Preparations of Tar.-Oleum Picis Liquidoe, oil of tar. A volatile oil distilled from tar, and containing a great variety of compounds, including hydrocarbons, phenols, paraffin, etc., among the phenols being creosote and carbolic acid. Dose: H., fl. 3 ss. -iv.; D., Mij.-x.

Unguentum Picis Liquidoe, tar ointment, containing fifty per cent. of tar.

Physiological Action of the Turpentines. - They are topical irritants, general stimulants, anti- 
septics, anthelmintics, and diuretics, when given internally. They become rapidly absorbed, and are eliminated chiefly by the kidneys, the skin, and bronchial mucous membranes, stimulating at the points of elimination. The oil of turpentine is the most active of the lot, being also expectorant, hæmostatic, antiferment, antiparasitic, and diaphoretic. Applied to the skin it is an irritant, varying in intensity with the manner in which it is applied; if with severe friction and its evaporation prevented, it may vesicate and produce a serious blemish. Small doses stimulate the heart and respiration, while large doses are gastro-intestinal irritants and depressants; toxic doses paralyze the nerve centres in the cerebrum, cord, and medulla. During the excretion of large doses the urinary apparatus becomes irritated and congested, sometimes accompanied by strangury and even hæmaturia.

Tar resembles the turpentines in its action, and, as it contains both creosote and carbolic acid, has some of the qualities of these.

Pitch is a cutaneous stimulant.

Therapeutics of the Turpentines.-They are given as stimulants, diuretics, and anthelmintics, in combination with linseed oil in colic. The Canada balsam is an ingredient of flexible collodion, and is itself applied to wounds, particularly of the coronet. They are given in conjunction with camphor, extract of belladonna, and ammonium carbonate, in bronchitis and other pulmonary affections; are also contained in many hoof ointments. 
Resin is used as a diuretic in both horses and cattle, usually combined with saltpetre. Externally it is a stimulant, and as resin cerate is applied to wounds, sores, etc. This last preparation is much used as a base for blistering ointments.

Oil of turpentine is given in indigestion, atonic diarrhœa, to relieve flatulence, in colic, in nasal gleet, catarrh, bronchitis, scarlatina, purpura, pulmonary hemorrhage; in cattle practice, in hoven, chronic diarrhœea and dysentery; in puerperal apoplexy - with carbonate of ammonia; in puerperal peritonitis; also in post-partum hemorrhage and "red water." As an anthelmintic, combined with tincture of chloride of iron, gentian or quassia, and linseed oil, after first clearing the bowel by a cathartic. For destroying the strongylus micrurus and strongylus filaria in the air passages of calves and lambs, it is injected intratracheally, together with $m_{x} .-x x$. each of carbolic acid, glycerin, and chloroform. It is also used as an antidote to phosphorus poisoning, but for this purpose must be old so as to contain ozone; the new oil is useless. Externally, the oil of turpentine is used as a counter-irritant for the horse; it does not react so powerfully upon the skin of cattle. It is applied to the abdomen in colic, enteritis, peritonitis, and other painful abdominal affections; to rheumatic swellings of joints, to sprains and strains of tendons, etc., after the acute symptoms have been relieved by hot fomentation. It is also used in erysipelas, ringworm, dry gangrene of dogs' ears, and in foot-rot of sheep and cattle; as an antiseptic to poisoned wounds, bites, and 13 
stings. For rectal enemata (against worms, in colic, etc.), several ounces in one quart of starch-water or linseed oil.

Turpentine vapors are prescribed in pulmonary affections, usually oil of turpentine, oil of tar, and compound tincture of benzoin, of each fl. $z$ ss., to each steaming.

Black oil is a favorite application to punctures of the horse's foot, poisoned wounds, etc.; also in footrot.

Terebene is the form in which turpentine is best used as a stimulant expectorant, in chronic cough, bronchitis, and other throat and lung diseases.

Tar and oil of tar are given in respiratory diseases (particularly chronic) in all animals. They are used externally in grease heels, eczema, scaly skin diseases, thrush, canker, foot-rot, etc.

\section{EMMENAGOGUES.}

Ferrum, iron, is used as a tonic emmenagogue. See under Ferrum.

Cantharis, cantharides, Spanish fly. See under Cantharides.

Quinine has considerable emmenagogue power, by direct action as a stimulant upon the uterus itself. Large doses as an ecbolic; smaller doses, frequently repeated, as an emmenagogue.

Acidum Boricum, boric acid, boracic acid, boric anhydride. Obtained from the action of $\mathrm{H}_{2} \mathrm{SO}_{4}$ upon 
borax. Colorless, pearly, lamellar crystals, of a feebly acid reaction, a bitter taste, and a sweetish aftertaste. Soluble in 25 parts of cold water, in 3 parts of boiling water, in 15 parts of alcohol, and in 5 parts of glycerin. It is a non-volatile antiseptic, equal in power almost to carbolic acid, and because of its nonirritant property may be frequently employed where other antiseptics are excluded. It is but seldom used internally, and then only in fermentative diarrhœa of young animals; occasionally as an emmenagogue. It is excreted by the kidneys, and hence may be used to exercise its antiseptic action on the urinary apparatus. Conjoined with starch it is used externally as a disinfectant powder, or as an ointment (ten per cent.), or saturated solution to foul wounds, ulcers, burns, sores; as a spray or gargle in sore throat, to check excessive salivary or pharyngeal secretion, to relieve the itching of erythema and eczema; also in catarrhal and purulent conjunctivitis. Lint and cotton are sometimes rendered antiseptic by it. See also "Borax," under Sodium. Dose: H. and C., 3 ij.-v.; D., gr. v.-xx.

Boroglyceride, made by heating 92 parts of glycerin with 62 parts of boric acid, is used as an antiseptic dressing for wounds, etc., same as boric acid.

Strychnia, strychnine. See under Nux Vomica.

Purgatives, particularly aloes, croton oil, etc., act by determining the blood to the pelvic viscera.

Sinapis, mustard, in poultices to lumbar and pelvic region. 


\section{TOPICAL AGENTS.}

Rubefacients, Vesicants, and Pustulants.

Sinapis, Mustard (syn.-Semen Erucæ). The seeds of Sinapis alba-English or yellow mustard; and of Sinapis nigra-black or German mustard; natural order Cruciferæ. Are annual plants. Different varieties grow wild in Europe and North America, and are frequently used to adulterate the better sorts.

Composition of Mustard.

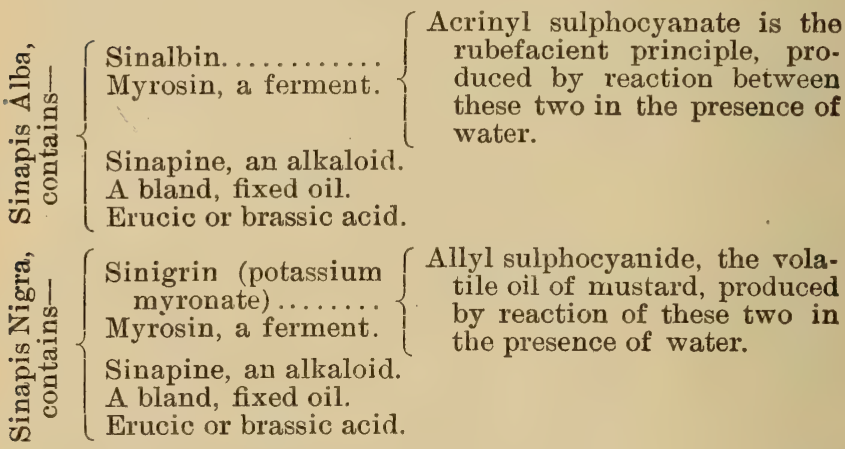

Mustard is used internally as a stomachic, carminative, stimulant, and emetic; slightly diuretic and laxative. Large doses are irritant. Externally as a rubefacient, vesicant, and suppurant. If mustard paste is rubbed upon the skin of a horse it causes redness, heat, tenderness, and some swelling in about twenty minutes. If allowed to remain on, vesication 
occurs in from two to six hours, and later these vesicles rupture and coalesce. If pure mustard has been used pustules may form, causing a sloughing of the skin and destruction of the hair roots. Mustard acts more promptly than cantharides, but its effects are not so permanent. It is the most suitable blister for cattle, but should not be used full strength for the dog or sheep. Mustard is used in catarrh, sore throat, laryngitis and pharyngitis, bronchitis, pneumonia, pleurisy, indigestion, colic, chronic rheumatic affections of the joints in cattle. In parturient apoplexy in the cow; sometimes applied over the kidneys to promote diuresis by counter-irritation. Its effects may be increased by adding a small amount of turpentine. The volatile oil of mustard is sometimes used to sharpen liniments, but must be cautiously used, not only on account of its great activity, but also because of its toxic properties.

Pulvis Sinapis Seminis, powdered mustard seed. Dose: H. and C., $3 \mathrm{ij}$ :-vi.; D., gr. x.-xx.; as an emetic, gr. $\mathrm{xxx} .-3 \mathrm{i}$, in warm water.

Pix Liquida and Oleum Picis Liquidoe. See under Turpentines, etc.

Camphora. See under Camphor.

Terebinthina. See under Turpentine.

Capsicum. See under Capsicum.

Cantharis, cantharides, Spanish fly. The dried insect, Cantharis vesicatoria, found in southern $\mathrm{Eu}$ rope. The insect has a predilection for lilac, ash, elder, honeysuckle, and rose bushes. It is gathered during the months of May and June, being destroyed 
at night by turpentine fumes. The flies are six to ten lines in length, two to three lines broad, and weigh one and one-half to two grains. The parts of their greenish, bronze-like wings are discernible in the powdered drug. Cantharides may be extracted by maceration in alcohol, ether, acetic acid, fixed and volatile oils. Its active principle-cantharidin-of which it contains about two per cent., occurs as colorless scales or crystals, insoluble in $\mathrm{H}_{2} \mathrm{O}$, but soluble in alcohol, ether, acetic acid, chloroform, and oils. The activity of Spanish fly is impaired by age. It must be kept in well-stoppered bottles.

Physiological Actions. - Cantharides is a powerful irritant, and produces its effect upon any part of the animal organism with which the free cantharidin is brought in contact. Externally applied it stimulates and vesicates, and is used as a counter-irritant. Taken internally it irritates the digestive mucous membranes, large doses producing violent gastro-enteritis, strangury, and hæmaturia.

Cantharides produce redness, vesication, and sloughing of the skin or mucous surfaces, in accordance to the strength of the preparation used and the length of time it is left on. The usual "cantharides blister," vesicates in from four to twelve hours; the vesicles soon burst and discharge a yellowish serous fluid, which must be carefully wiped away, as in many instances this also will vesicate. The surrounding parts should always, therefore, be protected by the use of lard, vaseline, etc. Cantharides is given internally in chronic cystitis and to prevent the invol- 
untary escape of urine from the bladder, due to relaxation of the sphincter vesicæ. It is also recommended for mares and cows slow in coming to service, but its aphrodisiac effects are uncertain. Externally, cantharidal ointments are applied to stimulate indolent wounds and ulcers, and to develop and determine slow-forming abscesses-as in strangles; also to help in the absorption and liquefaction of products of inflammatory processes; to open joint-the resulting inflammation and swelling sometimes check the escape of synovia. Similarly applied in umbilical hernia of foals and calves; as a counter-irritant to relieve irritability of larynx, chronic cough, and inflammation of deeper-seated structures; mild preparations to the chest walls in pleurodynia and pleurisy; along the spine in spinitis, chronic paralysis, encephalitis, puerperal apoplexy; also to rheumatic swellings of the articulations, especially if chronic. Inflammation of bursæ, tendons, articulations, ligaments, and bones are treated with cantharides preparations of varying strength. The usual ointment is twelve per cent., but much stronger may be employed if required.

Cantharides liniment (cantharides 3 iss., extracted by alcohol $\xi$ xiv., and oil of turpentine $\xi \mathrm{ij}$.), so-called "sweating blister," may be repeatedly used without blemishing.

A very good stimulant and absorbent ointment is composed of powdered cantharides $\xi$ iss., powdered camphor $\xi$ ss., oil of turpentine $\xi$ iss., biniodide of mercury $\xi$ i., and resin cerate $\xi$ xij.; to be made 
without tho aid of heat. Is ready for use in about ten to twelve days after preparation.

Euphorbium, an acrid resin obtained from the Euphorbia resinifera, natural order Euphorbiaceæ. Is a very energetic irritant, vesicant, and pustulant, but for horses and dogs is rather too unmanageable. An alcoholic solution (six per cent.) is said to be the better way of using it as a vesicant. 'It is, however, sometimes added to blistering ointments to increase their activity.

Oleum Tiglii. See under Croton Oil.

\section{Emollients AND Demulcents.}

Petrolatum, petroleum jelly, commonly called vaseline, cosmoline, etc. Is a jelly-like preparation, obtained from the residuum of petroleum and afterward purified. It is soluble in ether, chloroform, fixed and volatile oils; insoluble in alcohol and water. It is tasteless or nearly so, odorless, and of a neutral reaction. It is non-oxidizable, and hence does not become rancid, and therefore makes a most excellent basis for ointments. It is used as an emollient and lubricant; also as an excipient in making boluses and electuaries.

Mel, honey. A saccharine secretion deposited in the honeycomb by Apis mellifica, the honey-bee, an insect of the order Hymenoptera.

Mel Despumatum, clarified honey, is honey warmed, skimmed, and strained, with five per cent. of glycerin added.

Oxymel consists of honey 40, acetic acid 5, and water 5 parts. 
Linum, flaxseed or linseed.

Semen Lini, the dried seeds of Linum usitatissimum.

Oleum Lini, the oil expressed from the seeds. See under Oleum Lini.

Farina Lini, Semen Lini Pulvis, flaxseed meal, linseed meal. The ground seeds.

Linseed cake, the residue left after the oil has been expressed from the seeds, is used as a food for cattle and sheep in somelocalities, and in smaller quantities is fed also to horses. Linseed gruel or tea is nutritious as well as demulcent and lubricant; hence is given to animals recovering from debilitating diseases; also as a vehicle for medicines which might irritate the alimentary canal during their administration or action. Linseed meal is used as a poultice, retaining both heat and moisture very well. The oil is given for its cathartic qualities as well as its lubricant and demulcent action. See under Oleum Lini.

Adeps Suilli, lard; Adeps Lance, wool fat or lanolin, as well as most fixed oils (see under Oils and Fats), also answer the same purposes.

Glycerinum, glycerin, glycerol, prophenyl hydrate. A liquid obtained by the decomposition of fats or fixed oils, and containing not less than ninety-five per cent. of absolute glycerin $\left(\mathrm{C}_{3} \mathrm{H}_{5}[\mathrm{HO}]_{3}\right)$. It is a clear, colorless liquid, of syrupy consistence; oily to the touch, hygroscopic, odorless, very sweet and slightly warm to the taste, and neutral in reaction. It is soluble in all proportions in water and in alcohol, 
in a mixture of alcohol 3 with ether 1 , but insoluble in ether, chloroform, benzol, or fixed oils. Specific gravity not less than 1.250.

ACTIONS AND Uses.-Glycerin is chiefly demulcent and emollient; in large doses, undiluted, somewhat irritant to the local mucous membranes whether given per os or per rectum. It has some antiseptic and antiparasitic properties, but these are only slight. Enemata of fl. $\overline{3} \mathrm{ij} .-\mathrm{vi}$. are laxative for the horse; fl. $\overline{\text { ss. }}-\mathrm{i}$. for dogs. It forms the bases for many antiseptic and astringent preparations, and is added to liniments and lotions to prevent these from drying too rapidly. The chief glycerites are:

Glycerinum Acidi Carbolici, glycerin of carbolic acid, containing twenty per cent. of the acid.

Glycerinum Aluminis, glycerin of alum, contains fifteen per cent. of alum.

Glycerinum Tannini, glycerin of tannin, contains twenty per cent. of tannin.

Glycerinum Plumbi, glycerin of lead, contains fifty per cent. of Goulard's extract.

Glycerinum Amyli, glycerin of starch, contains starch 1, water 3 , and glycerin 5 .

The last of these, glycerin of starch, is of the consistence of petrolatum, and like the latter is used as an ointment base, being for this purpose cleaner than fatty bases. It can be readily washed off, but the therapeutist must not forget the incompatibility of starch with preparations of iodine. It is also a most excellent excipient in making pills or balls of quinine, etc. 


\section{MEMORANDA ON NEW REMEDIES.}

Aristol (syns.-Dithymoldiodide, Annidalin) is a somewhat unstable, reddish-brown, amorphous powder, insoluble in water and glycerin, slightly soluble in alcohol, very soluble in ether and in collodion. It is also taken up by fatty oils and vaseline when rubbed with these. It is prepared by the decomposition of a solution of iodine in iodide of potassium by means of an alcoholic solution of thymol, and contains about forty-six per cent. of iodine. Aristol was introduced as a substitute for iodoform, over which it has the advantage of being odorless. It is used in the same manner and for the same purposes as iodoform.

Formalin (formaldehyde, formic aldehyde, oxymethylene, formol), an aqueous solution of formaldehyde gas (from the oxidation of methyl alcohol), concentrated to forty per cent. $-\mathrm{CH}_{2} \mathrm{O}=\mathrm{H}$.CO.H. A clear, colorless liquid, having a peculiar pungent odor, non-irritant, non-toxic, non-corrosive; a powerful surgical and general antiseptic; used also as a food preservative $(1-1,000,000: 5,000)$. For hardening anatomical specimens, four to ten per cent. solution; in surgery, one-fourth to one-half per cent. solution.

Hydrogen dioxide $-\mathrm{H}_{2} \mathrm{O}_{2}$-a clear, colorless liquid, resembling in every way water, but differing from this in that it contains one more atom of O. Should be kept in well-corked bottles and in a cool place. Its strength is designated by volume-i.e., when vol- 
ume is spoken of in connection with $\mathrm{H}_{2} \mathrm{O}_{2}$ it means capacity for holding $\mathrm{O}$. One pint or unit of $\mathrm{H}_{2} \mathrm{O}$ one volume strong, contains one pint or unit of $\mathrm{O}$; while a fifteen-volume solution contains fifteen units of $\mathrm{O}$. When fresh and in good condition $\mathrm{H}_{2} \mathrm{O}_{2}$ is one of the most powerful oxidizing agents known, and in contact with pus forms a thick, white foam. Dose: H., fl. 3 ss.-ij.; D., fl. 3 ss.-i. Should be well diluted with water, milk, or gruel. Small repeated doses are preferable to a larger single dose.

It is given in choleraic complaints, dyspepsia, diarrhœa, and intestinal disorders accompanied by a tendency to fermentation and flatulence. It chccks fermentation and stimulates the gastric and intestinal fluids to a normal action. Diseases of the throat, nasal passages, and lungs may be treated with a spray of $\mathrm{H}_{2} \mathrm{O}_{2}, 1$ part in 5 to 15 of water. It is used also locally to wounds, sores, ulcers, fistulæ, punctured wounds of the feet, abscesses, and also in dentistry.

Piperazinum (syns. - Pyrazine hexahydride, di-ethylenediamine, piperazidine, ethylene-imine) $-\mathrm{C}_{4} \mathrm{H}_{10} \mathrm{~N}_{2}$-is a synthetical basic compound, formed by the action of ammonia upon ethylene bromide, and occurs as a white, crystalline powder, readily soluble in water. It liquefies when exposed to air, from which it greedily absorbs $\mathrm{H}_{2} \mathrm{O}$ and $\mathrm{CO}_{2}$. With uric acid it forms the most soluble known urate, requiring but 50 parts of water for its solution, while lithium urate requires 368 parts of water to dissolve it. Piperazin is non-toxic, non-irritant to mucous membranes, is readily absorbed from the stomach, and 
circulates in the blood unchanged. It has been recommended in rheumatism and azoturia. Dose: H., 3 i.-iij.; D., gr. v. -xv.

Barii Chloridum. See under Barium Chloride.

\section{PRESCRIPTION WRITING.}

Extemporaneous prescriptions are formulæ written by the veterinarian to meet the requirements of an individual case. They are divided into four parts ; viz. :

1. Superscription-consisting of a specification of the specie of the animal (i.e., whether horse, cow, or dog, etc.), and the owner's name, the date, and the sign $\mathrm{R}$ or Rp.-meaning Take! or Take thou!

2. Inscription-which is the real body of the prescription itself, and consisting of one or more of the following subdivisions: $(a)$ Basis or chief ingredient; (b) adjuvant, to assist the action of the basis; (c) corrective, to correct some injurious or undesirable quality of the other ingredients; $(d)$ excipient or vehicle, which give it form or bulk.

3. Subscription - the directions to the compounder, usually expressed in Latin.

4. Signature-the directions for the administration of the medicine, written in English or Latin, and the signature of the prescriber.

A prescription may contain but one or two ingredients, but nevertheless should be written out in the same manner. Simplicity is a very good point in prescribing, but a judicious blending of the actions of several drugs frequently gives a more decided and 
rapid curative effect. The above order should be followed out as nearly as possible, writing out first the names of all the ingredients, and then their quantity to make the correct dose. An example will be the following prescription:

$\left.\begin{array}{c}\text { New York, February 1st, 1896. } \\ \text { For Mr. Smith's Mare. }\end{array}\right\}$ Superscription.

R Aloes barbadensis .... 3 vij. - Basis.

Pulv.nucis vomicæ.... 3 i. -Adjuvant.

Pulv.capsici ........ 3 i

Fld. extr. belladonnæ.. 3 i.

Saponis moll.

Ut fiat bol. No. 1.

Sig. Purgative ball.

Give at once.

A. C. Hassloch, V. S. $\}$ Signature.

Words and Phrases in General Use in Prescription Writing.-The names of drug's should be written out as fully as possible, to avoid errors on the part of the compounder:

Adde, add.

Ana or āā, of each.

Bis, twice.

Cum, with.

Cola, strain.

Coletur, let be strained.

Dein, thereupon.

Detur, let be given.

Divide, divide.

Dividatur, let be divided.

Et, and.

Fac, make.

Filtra, filter.

Fiat or fiant, let be made.

Misce, mix.

Solve, dissolve.

Non, not.
In dies, daily.

Da, give.

Non-repetatur, not to be repeated.

Macera, macerate.

Ad saturandem, to saturation.

Quantum sufficiat (q. s.), as much as necessary.

In partes æquales, in equal parts.

Secundem artem, according to art.

Recipe $\mathrm{R}$, take.

Signa, write.

Pro re nata, according to need.

Ter, thrice.

Quartet, four times. 


\section{INCOMPATIBILITY.}

This may be either chemical, pharmaceutical, or therapeutical, according as the result is chemical decomposition, physical disassociation, or antagonistic physiological action. Simplicity in prescribing will to a great extent circumvent all of this.

The following are a few facts which may well be borne in mind so as to avoid incompatibility: Strong acids decompose salts of weaker acids, form ethers when combined with alcohol, and should not be combined with hydrates or carbonates. Potassium iodide decomposes metallic salts and is best given alone. Silver nitrate and the acetate and subacetate of lead, although incompatible with almost everything, combine well with opium. Tannin and gallic acid, and substances containing them, precipitate albumin, alkaloids, and most soluble metallic salts and gelatin. Iodine and iodides are incompatible with alkaloids and substances containing them.

Alkalies neutralize free acids; poisonous compounds are formed by the admixture of several substances-such as potassium iodide or syrup of iodide of iron, with potassium chlorate. Potassium cyanide or hydrocyanic acid dilute with calomel, bismuth salts, metallic hydrates, carbonates, subnitrates, or subchlorides, forming poisonous cyanides.

Explosive compounds result from mixing powerful oxidizing agents with others which are readily oxidizable. The chief members of these two classes are: 
Oxidizers. - Nitric acid, free hydrochloric acid, chromic acid, nitrohydrochloric acid, potassium chlorate, potassium permanganate.

Oxidizable or Combustible.-Glycerin, sugar, alcohols, oils and ethers, sulphur and sulphides, dry organic substances, phosphorus.

Pharmaceutical incompatibility differs from chemical incompatibility in the absence of chemical action, and is generally caused by adding one substance to another, which, through differences in solubility, cause a precipitation of solid matter or a separation of part of the liquid. Examples of pharmaceutical incompatibility are:

Resinous tinctures or fluid extracts with aqueous solutions. Essential oil with aqueous liquids in quantities exceeding 1 drop to $\xi i$. Tinctures made with alcohol with those made with diluted alcohol. Alcoholic tinctures and fluid extracts with aqueous preparations. Infusions generally, with metallic salts.

Therapeutical incompatibility arises when two agents are administered together which oppose each other in action on the system-as physostigmine with belladonna; morphine with atropine. The latter, however, are frequently used together, the one to act as a guard against excessive action of the other. See also under Antagonists of the various drugs, under their respective titles. 


\section{INDEX.}

PAGE.

Absolute Alcohol.............. 118

A. C. E. Mixture.................. 129

Acetanilid ...................... 161

Acetanilidum.................. 161

Acetate of Ammonia, solution of.

Acetate of Copper.

Acetate of Lead.................

Acetate of Morphine..............

Acetate of Potassium............

Acetate of Zinc ...................

Acetic Acid, Diliuted..............

Acetic Acid,' Pure..................

Acetum

Acetum Pịmbi................

Acid, Acetic, Diluted.............

Acid, Acetic, Pure................

Acid, Arsenious..................

Acid, Boracic ....................

Acid, Boric ......................

Acid Carbolic, Crude............

Acid, Carbolic, Glycerin of....163-202

Acid, Carbolic, Ointment of.... 163

Acid, Carbolic, Pure............ 162

Acid, Carbolic, solution of...... 163

Acid, Gallic..................... 81

Acid, Gallotannic................ 81

Acid, Hydrobromic, Diluted... 141

Acid, Hydrochloric............. 35

Acid, Hydrochloric, Dilutëd... 34

Aci 1, Muriatic ................ 35

Acid, Muriatic, Diluted.......... 34

Acid, Nitric.................... .

Acid, Nitric, Diluted............. 34

Acid, Nitro-Hydrochlorie....... 35

Acid, Nitro-Hydrochloric, Diluted

Acid, Phosphoric ..................

Acid, Phosphoric, Diluted......

Acids.

Acids, Mine.......................

Acid, Salieylic.......................

Acid, Sulphuric....................

Acid, Sulphuric, Aromatic......

Acid, Sulphuric, Diluted........

Acid, Tannic ...................

Acidum Aceticum Dilutum....

Acidum Aceticum Purum.......

Acidum Arseniosum ............

Acidum Boracicum.
Acidum Boricum

PAGE.

cidum Carbolicum Crudum.. 163

Acidum Carbolicum Purum

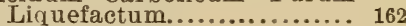

Acidum Gallicum............. 81

Acidum Hydrobromicum $\mathrm{Di}^{-}$ lutum................... 141

Acidum Hydrochloricum...... 35

Acidum Hydrochloricum Dilü-

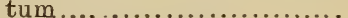

Acidum iuriäicum................

Acidum Muriaticum Dilutum. 34

Acidum Nitricum............ $\quad 35$

Acidum Nitricum Dilutum.... 34

Acidum Nitr o-Hydrochloricum..................... 35

Acidum Nitro-Hydrochloricum Dilutnm.............. $3 t$

Acidum Nitro-Muriaticum..... 35

Acidum Nitro-Muriaticum Di: lutum.

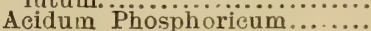

Acidum Phosphoricum Dilutum ...................... 35

Acidum Salieylicum............ 166

Acidum Sulphuricum........ 35

Acidum Sulphuricum Aroma-

ticum .....................

Acidum "Sulphurieum 'Dilütum ....................... 34

Acidum Tannicum............ 81

Acidifiers, Urinary.............. 24

Aconite........................ 150

Aconite, Fiuid Extract of...... 151

Aconite, Solid Extract of...... 150

Aconite, Tincture of........... 151

Aconite, Tincture of, Flem-

ing's ........................

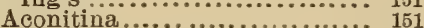

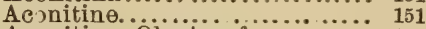

Aconitine, Oleate of ............. 151

Aconitum ..................... 150

Adeps .............................. 38

Adeps Lanä ..................... 201

Adeps Suilli...................... 201

Administration of Medicines.. $\quad 9$

Aerugo ...................... 86

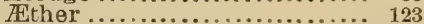

Atther Chioricus................... 129

Fther Fortior.................. 123 
Agents acting on Excretion... Agents acting on Microbes, etc. Ageuts acting on Parasites.... Agents acting on the Circulation

Agents acting on the Digestive Apparatus................... Agents acting on the Generative Apparatus...............

Agents acting on the Nervous system

Agents acting on the Respiratory Apparatus.............

Agents acting on the Skin ....... Agents asting upon Each Other ...................... Agents affecting Metabolism.. Agents affecting the Organs of Special Sense.

A pents Topical.................

19

Alcohol.....................118-119

Alcohol, Absolute............. 118

Alcohol Absolutum............. 118

Alcohol, Amylic................... 118

Alcohol, Diluted................ 119

Alcohol Dilutum ............... 119

Alcohol, Ethylic................. 118

Alcohol Methylic.............. 118

Alcoholic Extract of Belladonna Leaf.

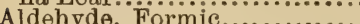

Alder, Black....................

A] $\theta$.

Alkalies .....................................

Alkalizers, Urinary..............

Alkaloids .......................

Aloë Barbadensis................

Aloë Capiensis..................

Aloë Socotrinae..................

Aloes, Barbadoes................. 175

Aloes, Cape................... 176

Aloes, Socotrine.............. 175

Alteratives.....................23, 70

Alteratives, Uterine............... 27

Alum.......................91, 172

Alum, Dried..................... ${ }_{92}$

Alum, Exsiccated............... 92

Alum, Glycerin of........... 202

Alumen ......................91, 172

Alumen Exsiceatum........... 92

Alumen Ustum.................. 92

American Hellebore........... 154

American Hellebore Root, fiuid extract of .....................

American Hellebore Root.

Tincture of................... 15t

American Wormseed............ 185

Ammonia, Aromatic, Spirit of. 62

Ammonia, Carbonate of........

Ammonia, Caustic...............

Ammonia, Concentrated........
Ammonia Liniment........... 62

Ammonia, Muriate of......... 62

Ammonia, Solution of Acetate

ef..... 62

Ammonia, stronger water of.. 61

Ammonia, Water of............ 61

Ammonii Bromidum............ 141

Ammonii Carbonas............ 62

Ammonii Chloridum............ 62

Ammonii Iodidum............. $7_{1}$

Ammonii Murias................ 62

Ammonium................... 61

Ammonium, Bromide of...... 141

Ammonium, Chloride of...... 62

Ammonium, Iodide of......... 71

Amvl Hydrate................. $11 \%$

Amyl Nitris ................. 158

Amyl, Nitrite of ............. 158

Amylic, Alcohol............. 118

Anaesthesia, Contra-indica-

tions for ................... 130

Anaesthetic Mixtures..........., 129

Anaestheties.................... 13

Anaesthetics, General ............ 13

Anaesth etics, Jocal ............13-14

Analgesies ................... 13

Analgesine ${ }^{\prime} . \ldots \ldots \ldots \ldots \ldots \ldots \ldots, 160$

Anaphrodisiacs............... 26

Anhidrotics................ 25

Anhydride, Boric............... 194

Annidalin ................... 203

Anodyne, Hoffmann's ......... 124

Anodynes..................... 13

Anodynes, General............ 13

Anodynes, Local................ 13

Antacids....................... 18

Antacids, Direct.............. 19

Antacids, Indirect.... . ..... 19

Antacids, Remo ${ }^{+} e . . . . . . . . . . \quad 19$

Antagonists................... 30

Anthelmintics.............22, 184

Antidotes..................... ${ }_{29}$

Antidotes for arsenical poison-

ing ....................... 47

Anti-emetics................ 19

Antifebrin .................. 151

Antilithics.................. 25

Antimonii et Potassae Tar-

tras......................... 172

Antimony and Potassium, Tar-

trate of ..................... 172

Antiperiodics.................24, 160

Antiphlogistics................ ${ }_{24}$

Antipyretics. $\quad . . . \ldots \ldots \ldots . .28,160$

Antipyrin.................... 160

Antipyrinum ................. 160

Antiseptics.................... ${ }_{29}$

Antisialics................... 18

Antispasmodies ............... 14

Antizymotics.................29, 160

A perients..................... 20 
Aphrodisiacs................ ${ }_{26}$

A pomorphina.............. 135, 172

A pomorphine.............. 135, 172

Aqua Ammoniae............. 61

Aqua Ammoniae forius...... 61

Aqua Calcis.................. 59

Aqua Camphora $0 . . . . . . . . . .111$

Aqua Fortis................. 35

Aqua Mentha Piperitae...... 170

Aqua Plumbi.................. 87

Aqua Regia................... $\quad 36$

Aquae .................... 3

Aqueous Extract of Opium.... 135

Aquila Alba................. 77

Areca Nut................... 184

Areca Nut, Powdered..... ..... 184

Arecae Semina............... 184

Argenti Nitras............... 84

Argenti Nitras Fusus......... 84

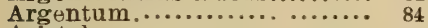

Aristol...................... 203

Arnica Flowers.............. 157

Arnica Flowers, tiucture of.... 158

Aromatic Spirit of Ammonia.. 62

Aromatic Sulphuric Acid..... 34

Arsenic.................... 45

Arsenic, White............... 45

Arsenical Poisoning, antidotes

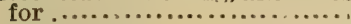

Arsenicum ................. 45

Arsenious Acid.............. 45

Arsenite of Potassium, Solu-

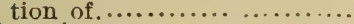

Arsenium ................... 45

Asafoetida .................. 113

Asafoetida, Emulsion of....... 114

Asafoetida, Tincture of....... 114

Asagroea Officinalis............ 154

Aspidium .................... 185

Astringents......................... 81

A stringents, Locail.................. 28

Astringents, Systemic......... 28

Atropina Sulphas............ 10.5

Atropine................... 104

Atropine, Derivatives of...... 104

Atropine, Sulphate of .......... 105

Auditory Nerve. Excitability

Baking sö........................... $\quad 15$

Ball........................

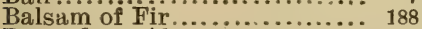

Barbadoes Aloes................ 175

Barii Chloridum............. . 182-205

Barium, Chloride of.........182, 205

Bark, Buckthorn............. 178

Bark, Calisaya............... 48

Bark, Cinchona, Powdered.... 49

Bark, Pale.................. 48

Bark. Peruvian................. 48

Bark, Red..................... 48

Bark, Sacred................. 178
Bark, White-Oak............ 83

Bark, Yellow.................. 48

Basilicon Ointmeut............ 190

Bean, Calabar.................147-148

Bean, Calabar, powdered...... 148

Beer........................ 120

Belladonna..................... 104

Belladonna Lèaf, powdered... 105

Belladonna Leaf, tincture of.. 105

Belladonna, Liniment of....... 105

Belladonna, Ointment of........ 105

Belladonna Root, fluid extract of ....................... 105

Belladonnine ..................... 104

Berries, Buckthorn............ 178

Biborate of Sodium........... 57

Bicarbonate of Sodium........... 56

Bicarbonate of Potassium ..... 53

Bichloride of Mercury......... 75

Biniodide of Mercury.......... 78

Bismuth.................... 45

Bismuth, Subearbonate........ 45

Bismuth, Subiodide........... 45

Bismuth, Subnitrate... ....... 45

Bismuthi Subnitras........... 45

Bismuthi Subcarbonas........ 54

Bismuthi Subiodidum........ 54

Bismuthum................. 45

Bisulphate of Quinine.......... 50

Bitartrate of Potassium........ 180

Bitter Tonics................. 47

Black Alder.................. 178

Black Mustard ................. 172

Black Uil...................... 180

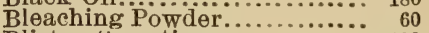

Blister, sweating................ 199

Blisters........................ ${ }^{27}$

Blue Mass....................... 783

Blue Ointment..................... 76

Blue Rocket..................... 150

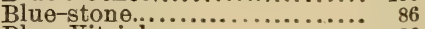

Blue Vitriol...................... 86

Bolus........................ 7

Boracic Acid...................... 194

Borate of Sodium.............. 57

Borax..................... 57

Boric Acid..................... 194

Boric Anhydride.............. 194

Boroglyceride................... 195

Bougia....................... 7

Bougies......................... 7

Brandy ........................ 11y

Brimstone................... 67

Bromide of Ammonium........ 141

Bromide of Calcium.......... 141

Bromide of Lithium............ 141

Bromide of Potassium .......53, 140

Bromide of Sodium ............ 141

Bromides.................. 140

Bromine and Bromides. . .... 140

Bromum ..................... 140 


\begin{tabular}{|c|c|c|}
\hline & PAGE. & \\
\hline Buchu, fluid extract of. & 187 & Capsulae \\
\hline $\begin{array}{l}\text { Foli: } \\
\text { Lea }\end{array}$ & $\begin{array}{l}186 \\
186\end{array}$ & $\begin{array}{l}\text { Capsules. } \\
\text { Carbolic Acid }\end{array}$ \\
\hline Buckthorn Bark & 178 & Carbolic Acid, C̈r \\
\hline thorn Berri & 178 & Carbolic Acid, Glycerir \\
\hline arn, Californ & 178 & Carbolic Acid, Ointmen \\
\hline lorns........ & 178 & Carbolic Acid, Pure.. \\
\hline gundy. & 190 & Carbolic Acid, Solution of ...... \\
\hline $\begin{array}{l}\ldots \ldots \\
\ldots \ldots \\
\ldots \ldots\end{array}$ & 39 & Carbolized Glycerite of Iodo- \\
\hline $\begin{array}{l}\text { acao Butt } \\
\text { affeina... }\end{array}$ & $\begin{array}{r}39 \\
186\end{array}$ & $\begin{array}{l}\text { Tannin. } \\
\text { Carbolized Iodine }\end{array}$ \\
\hline & & Car \\
\hline Bean. & 147 & Carbonate of Ammonia \\
\hline & 148 & te of Cal \\
\hline & 141 & oonate of Iron, $\mathrm{Ma}$ \\
\hline i Carb & 59 & bonate of Iron, $\mathrm{S}$ \\
\hline as. & & \\
\hline & 59 & ....... \\
\hline 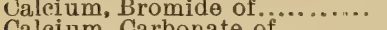 & 141 & $\ldots \ldots$ \\
\hline$f \ldots \ldots \ldots$ & 59 & of Soc \\
\hline , Oxide of............. Precipitated Phos- & 59 & $\begin{array}{l}\text { ate of Zir } \\
\text { Sedative }\end{array}$ \\
\hline & 59 & Stimular \\
\hline & 66 & hies.. \\
\hline ......... & 178 & ves ... \\
\hline & 48 & \\
\hline . 77, 183, & 186 & ada..... \\
\hline & 69 & ...........38, 174, \\
\hline$\cdots \cdots$ & 60 & $\mathrm{Ca}$ \\
\hline$\ldots \ldots \ldots \ldots \ldots$ & 66 & pound Tincture \\
\hline …........111, & 164 & apound Tincture \\
\hline d Tincture & & Cathartic \\
\hline & 11 & … 61 \\
\hline r, Liniment of ......... & 11 & tic, Lunar................... \\
\hline & 11 & \\
\hline le $\cdots 112$, & & $10 \mathrm{P}$ \\
\hline & 11 & $\mathrm{Ce}$ \\
\hline & 11 & \\
\hline$\ldots$ & 111 & \\
\hline $\begin{array}{l}\text {...111, } \\
\text {-..112, }\end{array}$ & & ants............12, 118 \\
\hline & & $\ldots \ldots \ldots \ldots \ldots 12,11$ \\
\hline & & \\
\hline ....... & 112 & \\
\hline$r \theta$ of & & ated.. \\
\hline & & 185 \\
\hline & 19 & \\
\hline & & 13 \\
\hline & & \\
\hline & & f Potassium..... \\
\hline & 19 & Chl \\
\hline & & m..........182, \\
\hline .....194, & 19 & Tincture of.. \\
\hline & & de of $\operatorname{Lim} \theta . . . .$. \\
\hline & & de of Mereury, Corros \\
\hline n or.......... & & um................... \\
\hline n......... & 187 & . $\ldots \ldots \ldots \ldots \ldots \ldots$ \\
\hline & & \\
\hline
\end{tabular}


Chlorine.................... 170

Chloroform................ 127

Chloroform, Commercial....... 128

Chloroform Liniment... ...... 128

Chloroform Liniment, Compound ......................

Chloroform, Purified ..........

Chloroform, Spirit of ...........

Chloroformum................

Chloroformum Purificatum...

Chloroformum Venale....... 12

Chlorum.................... 170

Cholagogue Purgatives.......21, 183

Cholagogues................ 21

Ciliary Excitants. ........... 16

Cinchona.................. 48

Cinchona Bark, Compound Tincture of ................

Cinchona Bark, Powdered.....

Cinchona, Composition of......

Cinchona, Compound Tincture

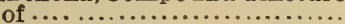

Cinchona Flava

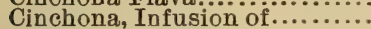

Cinchona Pallida ............

Cinchona, Preparations of....

Cinchona Rubra...............

Cinchonia

Cinchonidina.

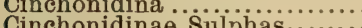

Cin hulphas.......

Cinchonidine, Sulphate of....

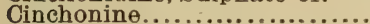

Citrate of Iron and Qüuinin $\Theta . .$.

Clarified Honey

Classification of Medicines.... 10

Clysters .................... 8

Cocainae Hydrochloras.......116 186

Cocainae Murias...............116, 186

Cocaine........................ 186

Cocaine, Hydrochiorate of ...116, 186

Cocaine, Muriate of...........116, 186

Codeina....................135, 136

Codeina Phosphas............... 137

Codeine ....................135, 136

Codeine, Phosphate of ......... 137

Cod-Liver Oil.................. 38

Colchici Radix................. 79

Colchici Radicis Pulvis.......... 80

Colchici Semen............... 79

Colchici Seminis Puivis.......... 80

Colchicina................... 80

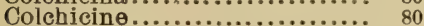

Colchicum..................... 79

Colchicum Root, Powdered... 80

Colchicum Seed, fluid extract of.

Colchicum Sëed, Powdered.... $\quad 80$

Collodia........................ 4

Collodion...................4, 124

Collodion, Flexible............. 124
Collodion, Styptic........... 124

Collodium.................... 124

Collodium Flexile............... 124

Collodium Stypticum......... 124

Colophony.................. 190

Collunarim................... 8

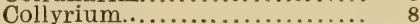

Commercial Chiloroform........ 128

Common Salt................. 56

Composition of Cinchona..... 48

Composition of Mustard....... 196

Compound Camphor Liniment.............. 112

Compound "Chloroform Liniment....................... 128

Compound Powder of $\mathrm{Rhu}$

barb .................... 179

Compound Solution of Iöline. 71

Compound Spirit of Fther.... 124

Compound Tincture of Camphor ...................... 112

Compound Tincture of Cinchona ...................... 50

Compound Tincture of Cinchona Bark. U.S.P.......... 50

Compound Tinet. of Gentian.. 48

Concentrated Ammonia...... 61

Contra-indications for anaes-

thesia...................... 130

Convallamarin................ 103

Convallamarinum............ 103

Convallaria.................... 103

Convallaria, fiuid extract of... 103

Coordination of Movement.... 14

Copper...................... 86

Copper, Acetate of .......... $\quad 86$

Copper Poisoning............. 86

Copper, Sulphate of...........86, 171

Copperas..................... 42

Corrosive Chloride of Mercury. $\quad 75$

Corrosive Sublimate........... $\quad 75$

Cosmoline.................... 200

Cotton-seed Öil.................. ${ }_{39}$

Cotton, Styptic.................. 43

Cream of Tartar.............. 180

Croton Oil .................... 1891, 200

Crude Carbolic Äid............ 163

Cupri Acetas.................. 86

Cupri Sulphas................86, 171

Cuprum ...................... 86

Cutch....................... 83

Deadly Nightshäe............ 104

Decocta .................... 4

Decoctions.................... 4

Deliriants........................ 12

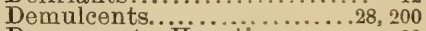

Depressants, Hepatic........... 22

Depressants, Renal........... 24

Depressants, Respiratory...... $\mathbf{1 5}$

Depressants, Uterine.......... 26

Derivatives of Atropine....... 104 
Diaphoretics............... 25

Diaphoretics, Nauseating.... . 25

Diaphoretics, Refrigerant.... 25

Diaphoretics, Simple......... 25

Di-ethylenediamine............. 204

Digestive Ferments........... 32

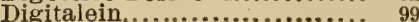

Digitalin..................... 99

Digitalinum................. 99

Digitalis....................98, 186

Digitalis, fiuid extract of..... 99

Digitalis, infusion of......... 99

Digitalis Leaf, powdered...... 99

Digitalis, Solid Extract of..... 99

Digitalis, Tincture of.......... 99

Digitin ....................... 99

Digitonin................... 99

Digitoxin..................... 99

Diluents..................... 25

Diluted Acetic A cid ............. 65

Diluted Alcohol............... 119

Diluted Hydrobromic Ä..... 141

Diluted Hydrochloric Acid.... 34

Diluted Nitric Acid............ 34

Diluted Nitro-hydrochloric

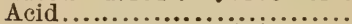

Diluted Phosphoric Acid........

Diluted Solution of Subacetate of Lead

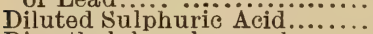

Dimethylphenylpyrazolon.....

Dioxide of Hydrogen...........

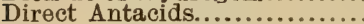

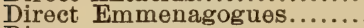

Discus.

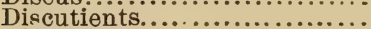

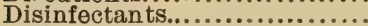

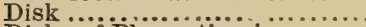

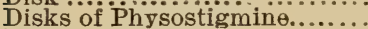

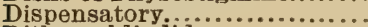

Dithymoldiodide............... 203

Diuretics.......................24, 186

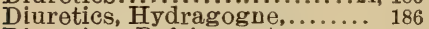

Diureties, Refrigerant........... 186

Diuretics, Stimulart.......... 186

Doses, Table of................ 31

Double hydrochlorate of Quinine and Urea...............

Douche, Nasal................... 8

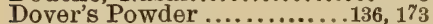

Drastic Purgatives............20, 181

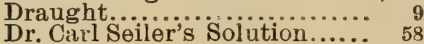

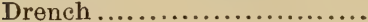

Dried Alum.......................

Dried Sulphate of Iron...........

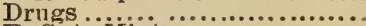

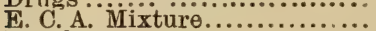

Ecbolics.......................

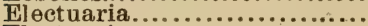

Electuaries....................
Elixir Paregoric.............. 135

Elixiria........................ 4

Emetics............................ 171

Emetics, General.............. 19

Emetics, Local.................. 19

Emetics, Systemic................ 19

Emmenagogues..............26, 194

Emmenagogues, Direct....... 26

Emmenagogues, Indirect...... 26

Emollients.................28, 200

Emplastra........................ 4

Emulsion of Asafoetida........ 114

Emulsiones.................... 8

Emulsions................... 8

Emulsum Äsafoetida............ 114

Endermic Method.............. 10

Enemata ..................... 8

Enepidermic Mëthod............ 10

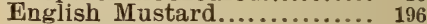

Epidermic Method.............. 10

Epispastics.................... 27

Epsom Salt........................ 179

Equilibrium, Määntenane of 14

Ergot...................... 96

Ergot, fluid extract of........... 97

Ergot of Rye................ 96

Ergot, powdered................ 97

Ergot, solid extract of......... 97

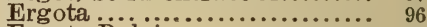

Ergota Pulvis..................... 97

Ergotin ........................ 97

Ergotole.................... 97

Erythroxylon Coca............ 116

Escharotics................... ${ }_{28}$

Eserinae Salicylas...........148, 182

Eserinae Sulphas............148, 182

Eserine, Salicylate of........148, 182

Eserine, Sulphate of........148, 182

Essence of Peppermint....... 170

Ether ....................... 123

Ether, (Jommercial............ 123

Ether, compound spirit of.... 123

Ether, nitrous, spirit of....... 125

Ether, stronger.............. 123

Ethyl Hydrate............... 118

Ethyl, Nitrite of.............. 159

Ethylen $\theta$-imine............... 204

Ethyli Nitris .................. 159

Ethylic Alcohol............... 118

Euphorbium................ 200

European Hellebore........... 154

Evacuents................... 171

Excitability of the Auditory

Nerve...................... 15

Excitants, Ciliary............... 16

Expectorants.................. 16

Expectorants, Nauseating.... 16

Expectorants, Stimulant...... 16

Extract, Goulard's............ 87

Extract of Aconite, fluid....... 151 

Extract of Aconite, solid....... Root, fluid. .................. Extract of Belladonna Leaf,

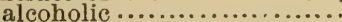
Extract of Belladonna Root,

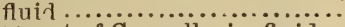
I xtract of Convallaria, fiuid... Extract of Digitalis, fluid...... Extract of Digitalis, solid......

Extract of Ergot, fluid.........

Extract of Ergot, solid.........

Extract of Gelsemium, fluid...

Extract of Gentian, fluid... . .

Extract of Henbane, fluid......

Extract of Henbane, solid......

Extruct of Indian Hemp, fluid.

Extract of Indian Hemp, solid.

Extract of Ipecac, fluid... ....

Extract of Jaborandi..........

Fxtract of Male Fern..........

Extract of Nux Vomica, fluid.. 94

Extract of Nux Vomica, solid.. 93

Extract of Opium, Aqueous.... 135

Extract of Pilocarpus, fiuid.... 146

Extract of Witch-Hazel....... 82

Extract, Pond's............... 82

Extracta.................. 4

Extracta Fluida.............

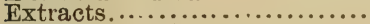

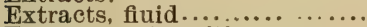

Extractum Aconiti..........

Extractum Aconiti Fluidum ...

Extractum Belladonnae Foliorum Alcoholicum.............

Extractum Belladonna Radicis Fluidum...............

Extractum Buchu Fluidum...

Extractum Cannabis Indica $\theta .$.

Extractum Cannabis Indicae Fluidum..................

Extractum Capsici Fluidum...

Extractum Colchici Seminis

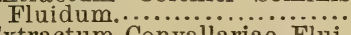

Extractum Convallariae Flui-

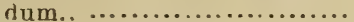

Extractum Digitalis.... ......

Extractum Digitalis Fluidum.

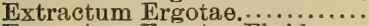

Extractum Ergotae Fluidum..

Extractum Gelsemii Fluidum.

Extractum Gentianae Flui-

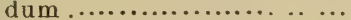

Extractum Hyoscyami.......

Extractum Hyoscyami Flui-

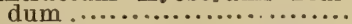

Extractum Ipecacuanhae Fluidum .....................

Extractum 'Jabörandi ' Fiui-

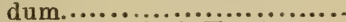

Extractum Nucis Vomicae...

4

3

5

82
4

4

4

Extractum Nucis Vomicae Fluidum................... 94

Extractum Opii.............. 135

Extractum Pancreaticus...... 33

Extractum Pilocarpi Fluidum. 146

Extratum Veratri Viridis Fluidum..................... 154

Eye-wash..................... 8

Faba Calabarica.............147, 148

Farina Lini..................... 201

Fats.......................... 38

Ferments, Digestive.......... 3.2

Fern, Male.................. 185

Ferri Carbonas Saccharatus... $\quad 42$

Ferri et Quininae Citras....... 51

Ferri Iodidum................ 42

Ferri Perchloridum.......... 43

Ferri Sulphas.............. . 42

Ferri Sulphas Exsiccatus..... 43

Ferro-chininum Citricam.... 51

Ferrum. ................. 41,194

Ferrum Reductum........... 41

Filix Mas.................... 185

Fir, Balsam of ... . ......... 188

Flaxseed .................... 201

Flaxseed Meal.... ........... 201

Flaxseed Oil................. 178

Fleming's Tincture of Aconite ..................... 151

Flexible Collodion................ 124

Flores Arnicae.................. 157

Flowers, Arnica.................. 157

Fluid Extract of Aconite........ 151

Fluid Extract of American Hellebore Root.............. 154

Fluid Extract of Belladonna Root ...................... 105

Fiuid Extract of Buchu........ 187

Fluid Extract of Capsicum.... 187

Fluid Extract of Colchicum Seed ....................... 80

Fluid Extract of Convallaria... 103

Fluid Extract of Digitalis...... 99

Fluid Extract of Ergot......... 97

Fluid Extract of Gelsemium... 144

Fluid Extract of Gentian....... 48

Fluid Extract of Henbane...... 110

Fluid Extract of Indian Hemp. 115

Fluid Extract of Inecac....... 173

Fluid Extract of Nux Vonica. 94

Fluid Extract of Pilocarpus... 146

Fluid Extracts................ 4

Fly, Spanish...................... 194, 197

Folia Buchu..................... 186

Folia Digitalis Pülvis........... 99

Fomentation................ 8

Foods........................... 22

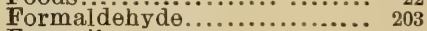

Formalin ..................... 203

Formic Aliẹhyde................ 203

Formol ..................... 203 
PAGE.

PAGF.

Formyl. Iodide of............ 72

Fotus.., 3 ..................... 8

Foxglove...................98, 186

Fused Nitrate of silver........ 84

Fusel Oil...................... 118

Galactagogues................ 27

Galactophyga................ 27

Galla........................ 81

Gallic Äeid......................... 81

Gallotannic Acid.............. 81

Gamboge ....................... 182

Gambogia.................... 182

Gargarysma................. 8

Gargle..................... 8

Gastric Sedatives, Local........ 19

Gastric Tonics................ 18

Gastro-intestinal koute...... 9

Gelsemina.................... 144

Gelsemine................... 144

Gelsemium, Fluid Extract of.. 144

Gelsemium.................. 144

Gelsemium, Tincture of...... 144

General Anaesthetics......... 13

General Anodynes.............. 13

General Emetics............... 19

General Gastric Sedatives..... 20

General sedatives............. 11

Gentian.......................... 47

Gentian, Compound Tincture

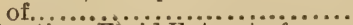

Gentian, Fluid Extract of.......

Gentiana......................

German Mistard................... 196

Gin....

Glauber's Salt.................. 7 , 181

Glonoin...................... 159

Glonoinum................... 159

Glucosidea................... 5

Glucosides.................... 5

Glycerin ..................... 201

Glycerin of Alum............ 202

Glycerin of Carbolic Acid...163, 202

Glycerin of Lead................ 202

Glycerin of Starch............ 202

Glycerin of Tannic Äcid........81, 202

Glycerines.................... 5

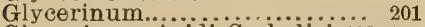

Glycerinum Acidi Carbolici.163, 202

Glycerinum Acidi Tannici....81, 202

Glycerinum Aluminis.......... 202

Glycerinum Amyli............. 202

Glycerinum Plumbi............ 202

Glycerinum 'I'annini Iodo-Carbolizati

Glycerita.................... 5

Glycerite of Iodo-Carbolate of Tannin .....................

Glycerites.......................

Glycerol.................... 201

Glyceryl, Trinitrate of......... 159

Gossypium Haemostaticum... 43

Goulard's Extract.............. 87

Grain Spirit .................... 118

Gray Powder.................77, 183

Green Vitriol....................... ${ }_{42}$

Gum Gutti..................... 182

Haematics....................... ${ }_{22}$

Hamamelis..................... 82

Hamamelis Virginica.......... 82

Hartshorn Liniment.............40, 62

Hartshorn Spirit.............. 61

Haustus..................... 9

Hellebore..................... 154

Hellebore, Ämerican........... 154

Hellebore, European........... 154

Hellebore, Mexican............ 154

Hemlock Pitch................ 190

Hemp, Indian.................. 115

Hemostatics .................... 28

Henbane....................... 109

Henbane, Fluid Extract of.... 110

Henbane, Solid Extract of..... 110

Henbane, Tincture of......... 110

Hepatic Depressants........... 22

Hepatic Stimulants............. 21

Hoffmann's Anodyne........... 124

Homatropine ................ 104

Honey ...................... 200

Honey, Clarified.............. 200

Horned Rye................... 96

Hydragogue Diiureties............ 186

Hydragogue Purgatives........ 20

Hydrarg yri Chloridum Corro-

sivum..................... 75

Hydrargyri C̈hloridum Mï̈̈..77, 183

Hydrargyri Iodidum Rubrum. 78

Hydrargyri Subsulphas

Flavus...................... 171

Hydrargyrum................ 75

Hydrargyrum cum Creta......77, 183

Hydrate of Chloral............. 131

Hydrate of Prophenyl........... 201

Hydrated Oxide of Iron........ 47

Hydrated Protoxide of Potash. 52

Hydrobromate of Hyoscine.... 110

Hydrobromic Acid, diluted.... 141

Hydrochlorate of Cocaine...... 116

Hydrochlorate of Morphine... 136

Hydrochlorate of Pilocarpine. 146

Hydroshlorate of Quinine.... 50

Hydrochloric Acid, Diluted.... 34

Hydrogen Dioxide............. 203

Hyoscinae Hydrobroma........ 110

Hyoscine, Hydrobromate of... 110

Hyoseyaminae Sulphas........ 110

Hyoseyamine, Sulphate of..... 110

Hyoscyamus.................. 109

Hypnotics..................... 12

Hypodermic Miethod............. 10

Hyposulphite of Sodium........ 66

Incompatibility............... 207 
Incompatibility, Pharmaceutical.... Incompatibility, Therapeutica Indian Hemp, fluid extract of. Indian Hemp, solid extract of. Indirect Antacids.

Indirect Emmenagogues....... Infusi

Infusion of Cinchona.......... Infusion of Digitalis.............

Infusions ........................ Infusum Cinchonae............ Infusum Digitalis.............. Inhalationes.

Inhalations ...........................

Injectiones.....................

Injections.

Injections Intra-arterial....... Injections, Intra-venous....... Injections, Parenchymatous.. Inoculation

Intra-arteriai Injections............ Intra-venous Injections......... Iodide of Ammonium............ Iodid $\theta$ of Formyl................. Iodide of Iron................... Iodide of Iron, Syrup of.......... Iodide of Potassium............53, 71 Iodide of Potassinm. Ointment of

Iodide of Sö Söium.....................

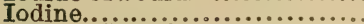

Iodine. Caribolized.................

Iodine, Ointment of..............

Iodine, Tincture of..............

Iodized Phenol...................

Iodoform .......................

Iodoformum ....................

Iodoform Ointment.............

Iodol.

Iodolum ......................

Iodo-phenyl...................

Iodo-tannin..................

Iodum ......................

Ipecac........................ pö of $\ldots \ldots \ldots \ldots \ldots \ldots \ldots \ldots \ldots \ldots 136,173$

Ipecac, fluid extract of....... 173

Ipecac, powdered.............. 173

Ipecac, Syrup of.............. 173

Ipecacuanha................. 172

Iron......................41, 194

Iron and Quinine, Citrate of.. 51

Iron, Dried Sulphate of ...... 43

Iron, Hydrated Oxide of........ 47

Iron, Iodide of ................ 42

Iron, Mass of Carbonate of... 45

Iron, Perchloride of ........... 43

Iron reduced by Hydrogen.... 41

Iron, Solution of Perchloride

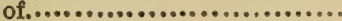

Iron, Saccharated Carbonate of........................ 42

Iron, Sulphate of........... 42

Iron, Syrup of Iodide of....... 42

Iron, Tincture of Chloride of.. 43

Irritants................... 27

Jaborandi.................. 145

Jalap......................... 181

Jalapa........................ 181

Jamaica Rum................. 120

Jervine ........................ 154

Juniper.................... 187

Juniper, Oil of............... 187

Juniperus.................. 187

Kino....................... 83

Kombé................... 102

Kutch................... 83

ILac Sulphur............... 66

Lactopeptin................. 33

Lamella $\Theta$ Physostigminae.... 148

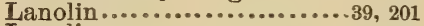

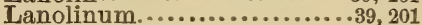

Lapis Infernalis.............. 84

Lard ..........................38, 201

Lard Oil..................... 39

Laudanum.................. 135

Laxatives....................20, 174

Lead........................... 87

Lead, Acetate of.............. 87

Lead and Opium, Solution of.. 88

Lead and Opium wash........ 88

Lead, Diluted Solution of Subacetate of..................... 87

Lead, Glycerin of............ 202

Lead, Solution of Subacetate of.......................... 87

Lead, sugar of............... 87

Lead Water................... 87

Leaf, Belladonna, alcohölic extract of.................. 105

Leaf. Belladonna, powdered.. 105

Teaf, Belladonna, Tincture of 105

Leaf, digitalis, powdered...... 99

Leaves, Buchu.................. 186

Levant Wormseed.................. 184

Lily of the Valley............... 103

Lime, Chloride of ............. 60

Lime, Chlorinatcd............ 60

Lime, Solution of............... 59

Lime, sulphurated............. 66

Lime Water................ 59

Liniment of $\ddot{A}$ mmonia..........40, 62

Liniment of Belladonna...... 105

Liniment of Camphor.......... 112

Liniment of Camphor, compound.................... 112

Liniment of Cantharides...... 199

Liniment of Chloroform....... 128

Liniment of Chloroform, compound......................

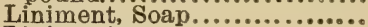

Linimenta....................
128

112 
PAGE.

Liniments............... 5

Linimentum Ammoniae........ 62

Linimentum Belladonnae..... 105

Linimentum Camphorae...... 112

Linimentum Camphorae Com-

position............................

inimentum Chloroformi.....

Linimentum Chloroform i Composition ................

Linimentum Saponis........... 112

Linseed....................... 201

Linseed Cäke.................... 201

Linseed Meal.................. 201

Linseed Oil....................... 178, 201

Liquid Tar. . . . . . . . . . . . . . . . . . 191

Liquor acidi carbolici.......... 163

Liquor Ammoniae............ 61

Liquor Ammonia fortior...... 61

Liquor Ammonii Acetatis..... 62

Liquor Calcis................ $\quad 59$

Liquores.....................

Liquor Ferri Perchloridi...... 43

Liquor Iodi Compositus....... 71

Liquor Morphinae Sulphatis,

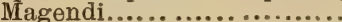

Liquor Morphinä Süulphatis, U. S P

(36

Liquor Pepsini.............. 33

Liquor Plumbi et Opii......... 88

Liquor Plumbi subacetatis.... 87

Liquor Plumbi Subacetatis dilutus.................... 87

Liquor Potassae.............. 52

Liquor Potassii Arsenitis...... 46

Lithii Bromidum.............. 141

Lithii Salieylas.................. 166

Lithium, Bromide of......... 141

Lithium, Salicylate of....... 166

Lithontriptics.............. 25

Local Anaesthetics..............13,14

Local Anodynes............... 13

Local Ástringents.............. 28

Local Emetics.................. 19

Local Gastric Sëatives........ 19

Local Sedatives.................. 11

Lotio......................... 9

Lotion 9

Lugols' Solution................ 71

Lunar Caustic................. 84

Magendie's Solution of Morphine.

Magnesia ànd R̈hübarb......... 179

Magnesii Carbonas............174, 179

Magnesii Sulphas............. 179

Magnesium, Carbonate of...174, 179

Magnesium, Sulphate of..... 179

Maintenances of Equilibrium. 14

Male Fern................. 185

Male Fern, Extract of......... 185

Male Fern, Oleoresin of ....... 185
PAGY.

Mass Blue................... 183

Mass of Carbonate of Iron..... 45

Mass, Vallets................. 45

Massa Ferri Carbonatis....... 45

Massa Hydrargyri............ 183

Materia Medica................ 1

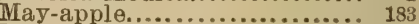

Meadow Saffron............... 79

Medication by respiratory

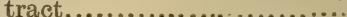

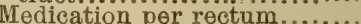

Medicines, Abministration of.

Medicines, Classification of .. 10

Mel......................... 200

Mel Despumatum............ 200

Menthol....................... 169

Mercurial Ointment............ 76

Mercuric Chloride............ $\quad 75$

Mercuric Iodide............... 78

Mercuric, subsuiphate, yëllow 171

Mercurous Chloride..........77, 183

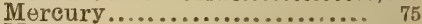

Mercury, bichioride of....... 75

Mercury, Biniodide of........... 78

Mercury, Corrosive Chloride of 75

Mercury, mild chloride of ....77, 183

Mercury, Oleate of ........... 77

Mercury, Red Iodide of ........ 78

Mercury, Subchloride of......77, 183

Mercury with Chalk..........77, 183

Method, Endermic................... 10

Method, Enepidermic.......... 10

Method, Epidermic................. 10

Method, Hypodermic........... 10

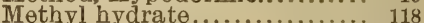

Methyl terchloride............ 127

Methylic Alcohol.................. 118

Mexican Hellebore............. 154

Mild Chloride of Mereury....77, 183

Milk of Sulphur.............. 66

Mindererus spirit............. 62

Mineral Acids.................... 34

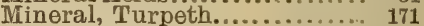

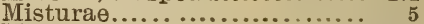

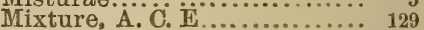

Mixture, E, C. A................. 129

Mixture, Nussbaums.......... 129

Mixtures...................... 5

Mixtures, anaesthetic......... 129

Monkshood.................. 150

Monobromated Camphor. ...112, 141

Morphina................... 134

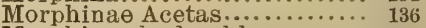

Morphinae hydrochloras. ...... 136

Morphina $\Theta$ Murias.............. 136

Morphinae sulphas............. 136

Morphine.................... 134

Morphine, acetate of .......... 136

Morphine, hydrochlorate of... 136

Morphine, Magendies' solution

of.......................... 136 
Morphine, Muriate of ......... 136

Morphine, preparations of...... 136 Morphine, solution of, U. S. P. 136 Morphine, sulphate of........ 136 Motor Depressants.............11, 144

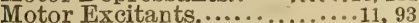
Movement, Coördination of.... 14 Mucilages. 5 Mucilagines................ 5 Muriate of Ammonia......... 62

Muriate of Cocaine........... 116 Muriate of Morphine.......... 136 Muriate of Pilocarpine........ 146 Muriate of Quinine.......... 50

Muriatic Acid................ 35

Mustard....................172, 195 Mustard, Black.............172, 196 Mustard, Composition of....... 196 Mustard, English............172, 196 Mustard, German............172, 196 Mustard Seed, powdered.....172, 197 Mustard, yellow..............172, 196 Mydriatics................... 14

Myotics.................... 15

Narceina.................. 135

Narceine..................... 135

Narcotics...................... 12

Narcotina...................... 135

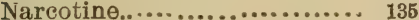

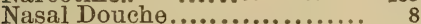

Nasal Wash.................. 8

Nauseating Diaphoretics....... 25

Nauseating Expectorants.... 16

New Remedies................. 203

Nightshade. deadly............. 104

Nitrate of Pilocarpine.......... 146

Nitrate of Potassium......... 53

Nitrate of Silver.............. 84

Nitrate of Silver. Füsed........ 84

Nitre...................... 53

Nitre, sweet spirits of ........125, 186

Nitric Acid................. 35

Nitric Acid, Diluted............ 34

Nitrite of Amy1................ 158

Nitrite of Ethyl................ 159

Nitrite of Potassium............ 159

Nitrite of Sodium,............. 159

Nitrites....................... 158

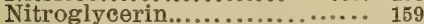

Nitro-hydrochloric Acid...... 35

Nitro-hydrochloric Acid, Diluted......................... Nitrous Ether, spirit of..........

Nussbaum's Mixture........... Nut, Areca................... Nut. Areca, powdered........... Nutgall.....................

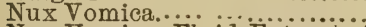
Nux Vomica, Fluid Extract of Nux Vomica, Powdered........ Nux, Vomica, solid extract of.
Nux Vomica, Tincture of..... 94

Ocular Sensibility............. 15

Official Preparations...........

Oil, Black.................. 189

Oil, Camphorated............. 112

Oil, Carbolized.................. 163

Oil, Castor.................39. 174, 170

Oil, Cod-liver.................. 38

Oil, Cotton-seed............... 39

Oil, Croton...................... 181, 200

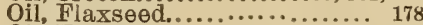

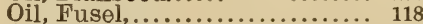

Oil of Juniper................. 187

Oil, Lard ...................... 39

Oil, Linseed..............39, 178, 201

Oil of Peppermint............ 169

Oil of Tar...................... 191

Oil of Turpentine...........185, 189

Oil of Turpentine, Rectified... 189

Oil of Vitriol................ 35

Oil of Wormseed............... 185

Oil, Olive ................... 39

Oils.......................... 38

Ointment, Basilicon........... 190

Ointment, Blue................. 76

Ointment, Mercurial.......... 76

Ointment of Ammoniated $\ddot{M}$ er-

cury..................... 77

Ointment of Belladonna...... 105

Ointment of Carbolic Acid..... 163

Ointment of Iodide of Potas-

sium...................... 72

Ointment of Iodine.......... 71

Ointment of Iodoform......... 72

Ointment of Tannic Acid..... 81

Ointment of Tar.................. 191

Ointment of Veratrine........ 155

Ointment of white precipitate. $\quad 77$

Ointment, Simple............ 7

Ointments................. 7

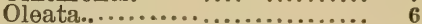

Oleate of Aconitine............. 151

Oleate of Mercury............. 77

Oleate of Veratrine.......... 155

Oleates.,.................... 6

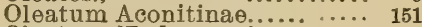

Oleatum Hydrargyri......... $\quad 77$

Oleatum Veratrinae........... 155

Oleoresin of Cansicum........ 187

Oleoresin of Male Fern........ 185

Oleoresina Aspidii............... 185

Oleoresina Capsici.............. 187

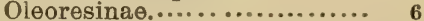

Oleoresins.................. 6

Oleum Adipis.................. $\quad 39$

Oleum Camphoratum........ 112

Oleum Carbolizatum,......... 163

Oleum Chenopodii............ 185

Oleum Crotonis..............181, 200

Oleum Gossypii seminis...... 39

Oleum Juniperi.............. 187 
Oleum Lini.............39, 178, 201

Oleum Menthae piperitae.... 169

Oleum Morrhua O............. $_{3} 38$

Oleum Nigrum................ 189

Oleum Olivae. ............... 39

Oleum picis liquidae.......... 191

Oleum Ricini.............39, 174, 178

Oleum Terebinthinae........185, 189

Oleum Terebinthinae Rectifi-

catum ..................... 189

Oleum Theobromae.......... 39

Oleum Tiglii................... 181, 200

Olive Oil.................... 39

Opii pulvis................... 135

Opium.................... 134

Opium and Ipecac, Powder of. 136

Opium, aqueous extract of ... 135

Opium, camphorated tincture

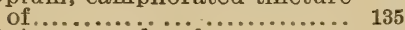

Opium, powdered............... 135

Opium, Tincture of .......... 135

Opodeldoc................... 112

Oxide of Calcium........... 59

Oxide of Zinc................. 89

Oxymel........................ 200

Oxymethylen $\theta . . . \ldots \ldots \ldots . . . . . .203$

Oxytocics..................... 26

Pale Bark...................... 48

Pancreatin.................... 33

Papain,..................... 32

Papaverina................... 135

Prpaverine................. 135

Papaw...................... 32

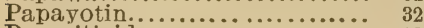

Parasiticides ................. 29

Paregoric.......................... 135

Parenchymatous Injections... 10

Pastes....................... 8

Pencils............................. 7

Pepper, Cayenne................. 187

Peppermint Camphor.......... 169

Peppermint, Essence of......... 170

Peppermint, Oil of............ 169

Peppermint, spirit of......... 170

Peppermint Water............ 170

Pepsin, pure.................. 32

Popsin, Saccharated.......... 32

Popsin, Solution of............. 33

Pepsinum Purum.............. $\quad 32$

Pepsinum Saccharatum...... 32

Perchloride of Iron........... 43

Perchloride of Iron, Solution of 43

Permanganate of Potassium.. 53

Peruvian Bark............... 48

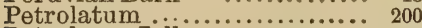

Petroleum Jelly.................. 200

Pharmaceutical Incompationil-

ity ..........................

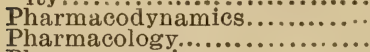

Pharmacopoeia.................

Pharmacy................... 1

Phøщаzone...................... 160

Phenol,....................... 162

Phenol, Iodized............... 72

Phenyl Acetamide................ 161

Phenyl, Salicylate of...........163, 166

Phosphate of Codeine........ 137

Phosphoric Acid.............. $\quad 36$

Phosphoric Acid, Diiluted....... 35

Physostigma.................. 147

Physostigminà salicylä.... 148, 182

Physostigmina $\theta$ sulphas....148, 182

Physostigmine, disks of...... 148

Physostigmine, salicylate $n$ " ' 8,182

Physostigmine, sulphate of. 1 , 182

Pills....................... 6

Pilocarpinae hydrochloras... 146

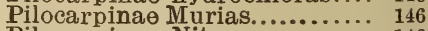

Pilocarpina Nitras............. 146

Pilocarpine, hydrochlorate of. 146

Pilocarpine, Nitrate of........ 146

Pilocarpine, Muriate of........ 146

Pilocarpus ..................... 145

Pilocarpus, Fiuid Extrait of.. 146

Pilula $. . . \ldots \ldots \ldots \ldots \ldots \ldots \ldots \ldots \ldots, 6$

Piperazidine........................ 204

Piperazine....................... 204

Piperazinum................... 204

Pitch......................... 188, 190

Pitch, Burgundy................ 190

Pitch, Canada.................. 190

Pitch, Hemlock................ 190

Pix.......................... 190

Pix Burgundica................ 190

Pix Canadensis................ 190

Pix Liquida ................... 191

Plasters...................... 4

Plumbi Acetas................... 87

Plumbum ...................... 87

Podophyllum ................... 183

Podophyllum, Rèsin of........ 183

Poke Root......................... 154

Poke Root, Powderej............. 155

Pond's Extract................ 82

Port Wine...................... 120

Porter....................... 121

Potash, Caustic.................. 52

Potash, hydrated protoxide of 52

Potassa..................... 52

Potassa, Solution of............. 52

Potassii Acetas................. 52

Potassii Bicarbonas.............. 53

Potassii Bitartras................. 180

Potassii Bromidum.............53, 140

Potassii Carbonas.............. 53

Potassii Chloras.............. 53

Potassii Iodidum............... 53, 71

Potassii Nitras.................. 53

Potassii Nitris.................. 159

Potassii Permangana........... 53

2 Potassium,................... 52 
PAGE.

PAGE.

Potassium, Acetate of........ 52

Potassium Alum.............. 91

Potassium, Bicarbonate of... 53

Potassium, bitartrate of....... 180

Potassium, Bromide of ........53, 140

Potassium, Carbonate of..... 53

Potassium, Chlorate of........ 53

Potassium, Iodide of.........53, 71

Potassium, Nitrate of.......... 53

Potassium, Nitrite of......... 159

Potassium, Permanganate of. 53

Potassium, Solution of Arsen-

ite of ..................... 46

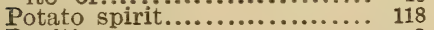

Poultices....................... 8

Powder, Dover's................36, 173

Powder, Gray................77, 183

Powder of Ipecac and Opium, 136,173

Powdered Áreca Nut......... 184

Powdered Belladonna Leaf.... 105

Powdered Calabar Bean....... 148

Powdered Camphor............ 111

Powdered Capsicum............. 187

Powdered Cinchona Bark.... 49

Powdered Colchicum Root.... 80

Powdered Colchicum Seed.... 80

Powdered digitalis leaf........ 99

Powdered Ergot............... 97

Powdered Gentian Root....... 48

Powdered Ipecac.............. 173

Powdered Mustard Seed........ 197

Powdered Nux Vomica......... 94

Powdered Opium............... 135

Powdered Poke Root........... 155

Powders...................... 6

Precipitated Chalk............ 59

Precipitated phosphäe of Cälcium...................... 59

Precipitated Sulphur.........66, 174

Preparations of Cinchona..... 49

Proparations, Official......... 3

Preparations of Morphine..... 136

Preparations, Unofficial...... 7

Prescription Writing.......... 205

Proof spirit.................. 120

Prophenyl hydrate............ 201

Protectives.................... 29

Pseudo-jervine.................... 154

Pulmonary Sedatives............ 15

Pulveres ..................... 6

Pulvis Arecà Seminis.......... 184

Pulvis Belladonnae foliorum. 105

Pulvis Corticis Cinchonae..... 49

Pulvis Gentianae............. 48

Pulvis Ipecacuanhae.......... 173

Pulvis Ipecacianha e et Öpii, 136, 173

Pulvis Magnesii cum Rheo.... 179

Pulvis Nucis Vomicae........ 94

Pnlvis Opii...................... 135

Pulvis Rhei Compositus....... 179

Pure Acetic Acid. . . ... . . ....... 64

Pure Carbolic Acid............. 162

Pure Pepsin.................... ${ }_{32}$

Purgatives ................... 20, 195

Purgatives, Chologogue.....21, 183

Purgatives, Drastic.............20, 181

Purgatives, Hydragogue... . 20

Purgatives, Saline............ 0, 179

Purgatives, Simple...........20, 175

Purging Buckthorn.......... 178

Purified Chloroform.......... 128

Pustulants ..................28, 196

Pyrazine Hexahydride........ 204

Quaker Button............... 93

Quercus albae cortex......... 83

Quercus lusitanica. ........... 82

Quicklime.................. 59

Quicksilver. .................. 75

Quinidina .................... 49

Quinidin $\theta \ldots . . \ldots \ldots \ldots \ldots \ldots \ldots .49$

Quininae Bimuriatis Carba-

mas...................... 50

Quininae Bisulphas...........50, 194

Quininae Hydrochloras.......50, 194

Quininae Murias.............50, 194

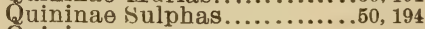

Quinine....................49, 194

Quinine and Iron, Citrate of. 51

Quinine and Urea, double

hydrochlorate of........... 50

Quinine Bisulphate...........50, 194

Quinine. Hydrochlorate of...50, 194

Quinine, Muriate of ..........50, 194

Quinine, Sulphate of ..........50, 194

Radix Colchici................. 79

Rectal Medication............... 9 9

Rectified Oil of Turpentine... 189

Rectified spirit............... 120

Red Bark...................... 48

Red Iodide of Mercury........ 78

Red Wine...................... 120

Refrigerant Diaphöröies....... 25

Refrigerant Diuretics......... 186

Refrigerants ................. 18

Remote Antacids............... 19

Renal Depressants.............. 24

Resin....................... 190

Resin Cerate.................... 190

Resina........................ 190

Resin of podophyllum......... 183

Resina Podophylli............. 183

Resinae...................... 6

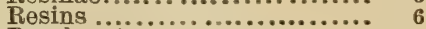

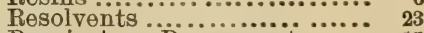

Respiratory Dëpressants...... 15

Respiratory Stimulants....... 15

Restorative Agents........... 32

Restoratives ....................... 22

Rhamnus Catharticus.......... 178

Rhamnus frangula........... 178

Pulvis Sinapis sominis,....... 197

Rhamnus Purshiana............ 


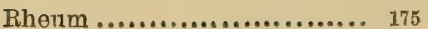

Rhubarb .................... 175

Rhubarb and M̈ìgnesia....... 179

Rhubarb, compound powder

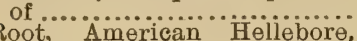
fluid extract of...............

Root, American Heilebore,

Tincture of................... of ........................

Root, Colchicum, Powdered..

Root, Poke....................

Route, Gastro-intestinal......

Rubefacients ...............27, 196

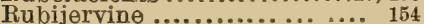

Rum, Jamaica................ 120

Rum, St. Croix.............. 120

Rye, Horned.................. 96

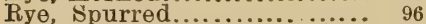

Saccharated Carbonate of Iron 42

Saccharated Pepsin........... 32

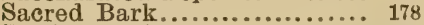

Sal Ammoniac................ 62

Salicylate of Eserine.........148, 182

Salicylate of Lithium.......... 166

Salicylate of phenyl..........163, 166

Salicylate of Physostigmine, 148, 182

Salicylate of Sodium.......... 166

Salicylic Acid.................. 166

Saline Purgatives..............20, 179

Salol......................163, 166

Salt, Common................ 56

Salt, Epsom................... 179

Salt, Glauber's.................57 181

Saltpotre .................. 53

Santonica .................... 184

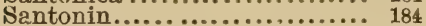

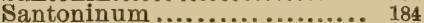

Secale Cornutum............. 96

Sedatives ...................... 11

Sedatives, Cardiac................ 17

Sedatives, Gastric, General... 20

Sedatives, General.................. 11

Sedatives, Local.............. 11

Sedatives, Local Gastric......... 19

Sedatives, Pulmonary......... 15

Sedatives, Urinary............. 25

Sedatives, Vascular.............. 17

Sedatives, Vesical............... 24

Seed, Colchicum, Powdered.. 80

Seed, Mustard, Powdered..... 197

Semen Colchici............... 79

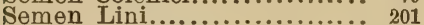

Semen Lini pulvis................. 201

Sense of Smell................ 15

Sensibility, Ocular.............. 15

She $\theta$ p's wool fat............... 39

Sherry ...................... 120

Sialogogues.................. 17
Silvor ................. 84

Silver, Nitrate of ................. 84

Silver, Nitrate of, Fused........ 84

Simple Diaphoretics........... 25

Simple Ointment............... 7

Simple purgatives..............20, 175

Sinapis.....................172, 195

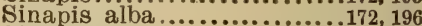

Sinapis Nigra..............172, 196

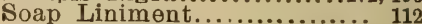

Soap spirit, camphorated...... 112

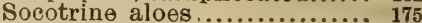

Soda Salaeratus................ $\quad \mathbf{5 6}$

Sodii Biboras.................. $\quad 57$

Sodii Bicarbonas.............. 56

Sodii Boras.................... 57

Sodii Bromidum................ 141

Sodii Carbonas.................. 56

Sodii Chloridum.............. 56

Sodii hyposulphis............. 66

Sodii Iodidum................ 71

Sodii Nitris................... 159

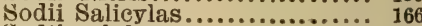

Sodii sulphas................57, 181

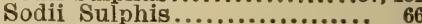

Sodii sulphocarbolas........... 163

Sodium ............. 163

Sodium, Biborate of.............. 57

Sodium, Bicarbonate of....... 56

Sodium, Borate of.............. 57

Sodium, Bromide of........... 141

Sodium, Carbonate of........... $\quad 56$

Sodium, Chloride of........... 56

Sodium hyposulphite........... 66

Sodium, Iodide of ............. $7_{1}$

Sodium, Nitrite of............. 159

Sodium, salieylate of........... 166

Sodium, sulphate of...........57, 181

Sodium sulphite................. 66

Sodium, sulphocarbolate of... 163

Solid Extract of Aconite....... 150

Solid Extract of Digitalis....... 99

Solid Extract of Ergot......... 97

Solid Extract of Henbane...... 110

Solid Extract of Indian Hemp 115

Solid Extract of Nux Vomica.. 99

Solution, Dr. Carl Seiler's.... 58

Solution, Fowler's.......... 46

Solution, Lugol's............. 71

Solution of Acetate of Ammo-

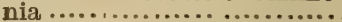

Solution of Arsenite of Potassium.................... 46

Solution of Carbolic Acid...... 163

Solution of Iodine, (Yompound 71

Solution of Iron Perchloride.. 43

Solution of Lead and Opium.. 88

Solution of Lime............. 59

Solution of Morphine Sulphate, Magendie's............ 136

Solution of Pepsin........... 33 
Solution of Potassa.......... 52 Solution of Subacetate of Lead 87 Solution of Subacetate of Lead, diluted................ Solution of Sulphate of Mor-

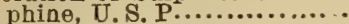

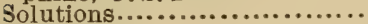

Spanish Fly..............194, 197

spirit Grain.................. 118

Spirit of Ammonia, Aromatic. 62

3pirit of Camphor ............ 111

Spirit of Chloroform......... 129

Spirit of Ether, compound.... 123

Spirit of Hartshorn .......... 61

Spirit of Nitre, sweet......... 125

Spirit of Nitrous Ether....... 125

Spirit of Peppermint......... 170

Spirit of Salt............... 35

Spirit of Turpentine.........185, 189

Spirit, Potato............... 118

Spirit, proof................. 120

Spirit, rectifled............... 120

Spirit, soap, camphorated.... 112

Spirit, wood................. 118

Spirits...$\ldots \ldots \ldots \ldots \ldots \ldots \ldots \ldots \ldots$

Spiritus ......................

Spiritus absolutus........... 118

Spiritus aetheris compositus. 124

Spiritus Aetheris nitrosi. .. 124, 186

Spiritus Ammonii Aromaticus 62

Spiritus Chloroformi......... 129

Spirjtus Camphora.......... 111

Spiritus Frumenti............ 119

Spiritus Glonoini............. 159

Spiritus Menthae piperita... .170

Spiritus Mindereri............ 62

Spiritus Nitrico-Dulcis......124, 186

Spiritus saponis camphoratus 112

Spiritus Vini Gallici........... 119

Spurred Rye................. 96

Starch, glycerin of........... 202

St. Croix Rum................ 120

stimulant Diuretics.............. 186

Stimulant Expectorants....... 16

Stimulants................ 10

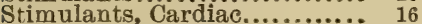

Stimulants, Hepatic............. 21

Stimulants, Respiratory........ 15

Stimulants, Vascular.......... 17

Stomachics.................. 18

Stout ...................... 121

Stronger Ether..................... 123

Stronger Solution of Ammonia 61

Stronger Water of Ammonia.. 61

Stronger White Wine......... 120

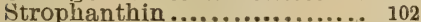

Strophanthinum .................. 102

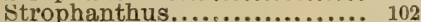

Strophanthus, tincture of.... 102

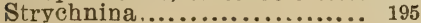

Strychninae Sulphas.........94, 195
Strychnine................. 195

Strychnine Poisoning......... 94

Strychnine, sulphate of......94, 195

Styptic Collodion................ 124

Styptic Cotton................... 43

Styptic, Warren's............. 38

Styptics ..................... 28

Subacetate of Lead, solution of 87

Subcarbonate of Bismuth.... $\quad 45$

Subchloride of Mercury...... 77

Subiodide of Bismuth......... 45

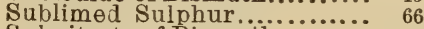

Subnitrate of Bismuth........ 45

Sudorifics.................... 25

Sugar of Lead................. 87

Sulphate of Atropine............ 105

Sulphate of Cinchonidine...... 50

Sulphate of Copper..........86, 171

Sulphate of Eserine................. 148

Sulphate of Hyoscyamine..... 110

Sulphate of Iron............. 42

Sulphate of Iron, Dried........ 43

Sulphate of Magnesia.................. 179

Sulphate of Morphine........... 136

Sulphate of Physostignine.... 148

Sulphate of Quinine.......... 50

Sulphate of Sodium............57, 181

Sulphate of Strychnine ............ 94

Suiphate of Zinc..............89, 171

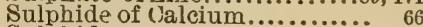

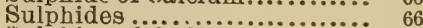

Sulphite of Söjium................ 66

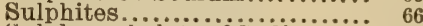

Sulphocarbolate of Sodium.... 163

Sulphur................... 66

Sulphur Lotum....................66, 174

Sulphur, Milk of................ 66

Sulphur, precipitated........66, 174

Sulphur Praecipitatum......66, 174

Sulphur, Sublimed............ 66

Sulphur, Washed...............66, 174

Sulphurated Lime.............. 66

Sulphuric Acid.................

Sulphuric Acid, Aromatic..... 34

Sulphuric Acid, Dilute........ $\mathbf{3 4}$

Suppositoria................ 6

Suppositories.................... 6

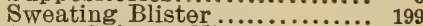

Sweet Spirit of Nitre..........125, 186

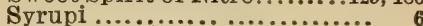

Syrup of İodide of Iron........

Syrup of Ipecac................. 173

Syrups ..................... 6

Syrupus Ferri İodidi............ 42

Syrupus Ipeca.cuanhae........ 173

Systemic Astringents.......... 28

Systemic Emetics.............. 19

Table of Doses................ 31

Tannic Acid..................... 81

Tannic Acid, Glycerin of.......81, 202

Tannic Acid, Ointment of.... 81 
Tannin ................ 81

Tannin, glycerin of iodo-car-

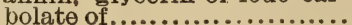

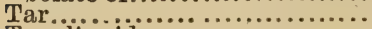

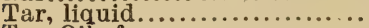

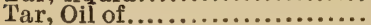

Tar, Ointment of ..............

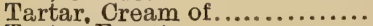

Tartar Emetic ............... tassium ..................

Tartarus Stibiatus.............

T'erebene....................

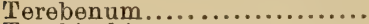

Terebinthina ..................

Terebinthina Canadensis....

'I'erebinthina Veneta...........

Tetra-iodo-pyrol .............

Trebaina.....................

Thebaine .....................

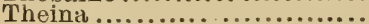

Theine ...................

Therapeutical Incompatibility

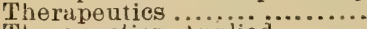

Therapeutics, Applied..........

'Therapeutics, Natural..........

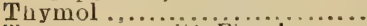

I'inctura aconiti, Fleming.....

Tiuctura Aconiti, U. S $\mathrm{F}$.......

I'inctura Arnicae flurum......

Tinctura Asafoetidae........

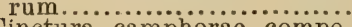

Tinctura camphorae composita.........................

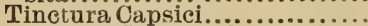

Tinctura Catechu Composita. .

Tinctura Cinchonae Compo-

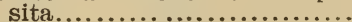

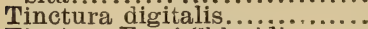

Tinctura Ferri Chloridi........

Tinctura Gelsemii...............

Tinctura Gentianae Compo-

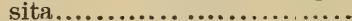

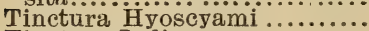

Tinctura Iodi..................

Tinctura Nucis Vomicae......

Tinctura Opii.................

Tinctura Upii Camphorata....

Tinetura Strophanthi.........

Tinctura Trinitrini..............

Tinctura Veratri Viridis.........

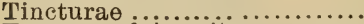

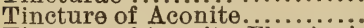

Tincture of Aconite, Fleming's

Tincture of American Helle-

bore Root...................

Tincture of Arnica flowers....

Tincture of Asafoetida........ 114

Tincture of Belladonna leaf... 105
PAGE.

Tincture of Camphor, Compound ..................... 112 Tincture of Capsicum........... 187 Tincture of Catechu, Compound ................. 83

Tincture of Chloride of Iron.. 43

Tineture of Cinchona Bark, Compound ................... 50

Tincture of Digitalis.............. 99

Tincture of Gelsemium........ 144

Tincture of Gentian, iompound ....................... 48

Tincture of Henbane............ 110

Tineture of Iodin $\theta . . . . . . . . . .6 .71$

Tincture of Nux Vomica...... 94

Tincture of Opium............ 135

Tincture of Opium, Camphorated....................... 135

Tincture of Strophanthus..... 102

Tinetures ..................... 7

Tonies........................ 23

Tonies, Cardiac................. 17

Tonics, Gastric................. 18

Tonies, Uterine................ 27

Tonies, Vascular............. 17

Tonics, Vesical.................. 24

Topical Agents................ 196

Toxicology ................... 1,2

Triuitrate of Gilyceryl.......... 159

Trinitrin ..................... 159

Turpentine.................... 188,197

Turpentine, Cinada........... 188

Turpentine, Oil of.........185, 189, 197

Turpentine, Oil of, rectified.189, 197

Turpentine, Spirit of....185, 189, 197

Turpentine, Venice............ 189

Turpeth Mineral............... 171

Unguenta................ 7

Unguentum Acidi Carbölici... 163

Unguentum Acidi Tannici.... 81

Unguentum Basiliconis....... 190

Unguentum Belladonnae...... 105

Unguentum Hydrargyri...... 76

Unguentum Hydrargyri Ammoniati.................... 77

Unguentum Iodi.................. 71

Unguentum Iodoformi......... 72

Unguentum Picis Liquida..... 191

Unguentum Potassii Iodidi... 72

Unguentum Simplex.......... 7

Unguentum Veratrinae........ 155

Unofficial Preparations........ 7

Urea and Quinine, double hy-

drochlorate of ...............

Urinary Acidifiers.............

Urinary Alkalizers.............

Urinary Sedatives...............

Uterine Alteratives..............

Uterine Depressants...........

Uterine Tonics ............... 
Vallet's Mass................ 45

Vapores ...................... 9

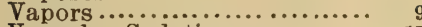

Vascular Sedatives............ 17

Vascular Stimulants.......... 17

Vascular Tonies.............. 17

Vaseline .................... 200

Venice Turpentine ............ 189

Veratralbine.................. 154

Veratrina .................... 155

Veratrine ..................154, 155

Veratrine, Ointment of........ 155

Veratrine, Oleate of ............ 155

Veratroidine ................. 154

Veratrum.................... 154

Veratrum Alhum............ T54

Veratrum Sabadilla........... 154

Veratrum Viride.............. 154

Veratrum Viride Pulvis...... 155

Verdigris...................... ${ }^{86}$

Vermicides......................

Vermifuges .................... 22

Vesical Sedatives............... 24

Vesical Tonies.................... 24

Vesicarts......................27, 196

Vina ....................... 7

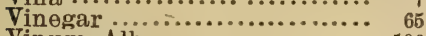

Vinum Album................ 120

Vinum album fortius.......... 120

Vinum Portense............... 120

Vinum Rubrum............... 120

Vinum Xericum............... 120

Vitriol, Blue.................. 86

Vit iol, Green ................. 42

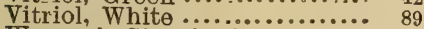

Warren's Styptic................ 38

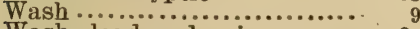

Wash, lead and opium........... 88

Wash, Nasal................... 8

Washed Sulphur................66, 174

Washing Soda.................. 56

Water, Camphor............... $11_{1}$

Water, load................${ }^{11}$
Water of Ammonia........... 61

Water, Peppermint........... 170

Waters....................... 3

Whiskey .................. 119

White Arsenic ............. 45

White Mustard ............... 172

White-oak Bark.............. 83

White Precipitate Ointment.. 77

White Vitriol................. 89

White Wine.............. .120

White Wine, stronger......... 120

Wine, Port.................. 120

Wine, Red ................ 120

Wine, Sherry ... ............ 120

Wine, White................. 120

Wine, White, stronger........ 120

Wines....................... 7

Witch-Hazel ................. 82

Witch-Hazel, Extract of..... 82

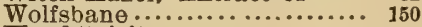

Wood spirit.................. 118

Wool Fat.................... 201

Wormseed, American........... 185

Wormseed, Levant.............. 184

Wormseed, Oil of.............. 185

Yellow Bark................... 48

Yellow Jasmine............... 144

Yellow Mustard................. 196

Yellow mercuric subsulphate. 171

Yellow Mustard............... 172

Zine........................... 89

Zine, Acetate of ................ 89

Zine, Carbonate of............... 89

Zine, Chloride of.............. 89

Zine, Oxide of ................. 89

Zinc, Sulphate of .............89, 171

Zinei Acetas.................. 89

Zinci Carbonas............... 89

Zinci Chloridum.............. 89

Zinci Oxidum................. 89

Zinci Sulphas.................89, 171

Zincum...................... 89 



\section{WILLIAM R. JENKINS, CATALOGUE 1896}

Any of these Books will be sent, Post Paid, on receipt of Price.

(*) Single asterisk designates New Books.

(**) Double asterisk designates Recent Publications.

A NDERSON. "Vice in the Horse" and other papers on Horses and Riding. By F. L. Anderson. Demy, 8 vo, eloth................................

ANDERSON. " How to Ride and school a Horse." With a System of Horse Gymnastics. By Edward L. Anderson. Cr. $8 v 0 \ldots \ldots \ldots \ldots \ldots \ldots \ldots \ldots$

$\left({ }^{* *}\right) \boldsymbol{B} \boldsymbol{A} C \boldsymbol{C H}$. "How to Judge a Horse." A concise treatise as to its Qualities and Soundness; Including Bits and Bitting-Saddles and Saddling, Stable Drainage, Driving One Horse, a Pair, Four-in-hand, or Tandem, ete. By Captain F. W. Bach. 12mo, cloth, fully illustrated $\$ 100$; paper............................50

BANHAM. "Tables of Veterinary Posology and Therapeutics," with weights, measures, etc. By George A. Banham, F.R.C.V.S. 12mo, cloth......75

BAUCHER. "Method of Horsemanship." Including the Breaking and Training of Horses.......... 00 
BELL. "The Veterinarians Call Book (Perpetual)," By Roscoe R. Bell, D V.S., Profsssor of Materia Medica, Therapeutics and Hygiene in the American Veterinary College, New York; President of the Long Island Veterinary Society; late U. S. Goverment Veterinary Inspector, etc.

A visiting list, that can be commenced at any time and used until full, containing much useful information for the student and the busy practitioner. Among contents are items concerning: Veterinary Drugs ; Poisons; Solubility of Drugs ; Composition of Milk,Bile, Blood, Gastric Juice, Urine, Saliva; Respiration; Dentition; Temperature, etc., etc. Bound in leather............................... 25

(")BRADLEY. " Outlines of Veterinary Anatomy." PART I.: The Anterior and Posterior Limbs. By O. Charnock Bradley, Member of the Royal College of Veterinary Surgeons; Professor of Anatomy in the New Veterinary College, Edinburgh. (Ready in June.) $12 \mathrm{mo}$, cloth, 189 pages..................

CLEMENT. "Veterinary Post Mortem Examinations." By A. W. Clement, V.s. Records of autopsies, to be of any value, should accurately represent the appearances of the tissues and organs so that a diagnosis might be made by the reader were not the examiners conclusions stated. To make the pathological conditions clear to the reader, some definite system of dissection is necessary. 'The absence in the English language, of any guide in making autopsies upon the lower animals, induced Dr. Clement to write this book, trusting that it would prove of practical value to the profession. $12 \mathrm{mo}$, cloth, illustrated ...................75 
851-853 Sixth Avenue (cor. 48th St.), New York. 3

(**)CADIOT. "Roaring in Horses." Its Pathology and Treatment. This work represents the latest development in operative methods for the alleviation of roaring. Each step is most clearly defined by excellent full-page illustrations. By P. J. Cadiot, Professor at the Veterinary School, Alfort. Trans. Thos. J. Watt Dollar, M.R.C.V.S., etc. Cloth....75

CHAUVEAU. "The Comparative Anatomy of the Domesticated Animals." By A. Chauveau. New edition, translated, enlarged and entirely revised hy George Fleming, F.R.C.V.S. 8vo. cloth with 585 Illustrations ........................ .5 75

CLARKE. "Horses' Teeth." A Treatise on their Anatomy, Pathology, Dentistry, etc. Revised and enlarged. By W. H. Clarke. 12mo, cloth......2 50

CLARKE. "Chart of the Feet and Teeth of Fossil Horses." . .......................25

\section{CLEAVELAND. "Pronouncing Medical Lexicon."}

Pocket edition. Cloth.....................75

COURTNEY. "Manual of Veterinary Medicine and Surgery." By Edward Courtney, V.S. Crown, 8vo, cloth................................2 75

(**) COX. "Horses: In Accident and Disease." The sketches introduced embrace various attitudes which have been observed, such as in choking; the disorders and accidents occurring to the stomach and intestines; affection of the brain ; and some special forms of lameness, etc. By J. Roalfe Cox, F.R.C.V.S. 8vo, cloth, fully illustrated ......................... 50 
CURTIS. "Horses, Cattle, Sheep and Swine." The origin, history improvement, description, characteristics, merits, objections, etc. By Geo. W. Curtis, M.S.A. Superbly illustrated. Cloth, $\$ 200$; half sheep, $\$ 2.75$; half morocco................ 50

DALZIEL. " British Dogs." Describing the History Characteristics, Points, and Club Standards, etc., etc. With numerous colored plates and wood engravings. By Hugh Dalziel. Vol. I., \$400. Vol. II., 8vo.400

DALZIEL. "The Fox Terrier." Illustrated. (Monographs on British Dogs)................... 00

DALZIEL. "Fox Terrier Stud Book." Edited by Hugh Dalziel.

Vol. I. Containing Pedigrees of over 1,400 of the bestknown Dogs, traced to their most remote known ancestors ............................... 00

Vol. II. Pedigrees of 1,544 Dogs, Show Record, \&c.1 00

Vol. III. Pedigrees of 1,214 Dogs,Show Record, \&c.1 00

Vol. IV. Pedigrees of 1,168 Dogs, Show Record, \&c.1 00

Vol. V. Pedigrees of 1,662 Dogs, Show Record, \&c.1 00

DALZIEL. "The St. Bernard." Illustrated...100

DALZIEL. "st. Bernard Stud Book." Edited by Hugh Dalziel.

Vol. I. Pedigrees of 1,278 of the best-known Dogs, traced to their most remote known ancestors, Show Record, \&c.............................. 00

Vol, II. Pedigrees of $564 \mathrm{D}$ ggs, Show Record, \&c..1 00 
851-853 Sixth Avenue (cor. 48th St.), New York. 5

DALZIEL. “The Diseases of Dogs." Their Pathology, Diagnosis and Treatment, with a dictionary of Canine Materia-Medica. By Hugh Dalziel 12mo, paper,

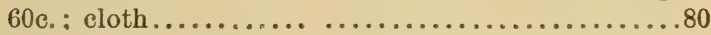

DALZIEL. “Diseases of Horses.” 12mo, cloth. .1 00

DALZIEL. "Breaking and Training Dogs." Being concise directions for the proper education of dogs, both for the field and for companions. Second edition, revised and enlarged. Part I, by Pathfinder: Part II, by Hugh Dalziel. 12mo, cloth, illus ....2.60

DALZIEL. "The Collie." Its History, Points, and Breeding. By Hugh Dalziel. Illustrated, 8vo, paper, 50c., cloth............................ 00

DALZIEL. "The Greyhound." 8vo, cloth, illus..1 00

$\boldsymbol{D A N C E}$. " Veterinary Tablet." Folded in cloth case. The tablet of A. A. Dance is a synopsis of the diseases of horses, cattle and dogs with the causes, symptoms

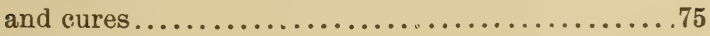

$\boldsymbol{D A N A}$. "Tables in Comparative Physiology." By Prof. C. L.Dana, M.D......................25

$\boldsymbol{D A} \boldsymbol{Y}$. "The Race-horse in Training," By Wm. Day, 8 vo ................................... 50

$\left.{ }^{* *}\right) D U N$. "Veterinary Medicines, Their Actions and Uses." By Finlay Dun, V.S. Revised edition (almost entirely re-written) 8 vo, cloth................ 50

DWYER. "Seats and Saddles." Bits and Bitting, Draught and Harness and the Prevention and Cure of Restiveness in Horses. By Francis Dwyer. Illustrated, 1 vol., $12 \mathrm{mo}$, oloth, gilt. .............. 50 
FLEMING. "A Treatise on Practical Horseshoeing."

By George Fleming, M.R.C.V.S. Cloth.........75

(") FLEMING. "Veterinary Obstetrics." Including the Accidents and Diseases incident to Pregnancy, Parturition, and the early Age in Domesticated Animals. By Geo Fleming, F.R.C.V.S. With 212 illustrations. New edition revised, 236 illustrations, 758 pages. ..6 25 773 pages, 8 vo, cloth (old edition) ............. 50

FLEMING. "Rabies and Hydrophobia." History. Natural Causes, Symptoms and Prevention. By Geo. Fleming, M. R.C.V.s. 8vo, cloth............. 75

FLEMING. "Propagation of Tubereulosis." Stating Injurious Effects from the consumption of the Flesh and Milk of Tuberculous Animals. By Geo. Fleming, M.D., M.R.C.V.S., and others. 8vo, cloth......1 50

FLEMING. "Tuberenlosis." From a Sanitary and Pathological Point of Vier..................25

FLEMING. "The Contagious Diseases of Animals." Their influence on the wealth and health of nations.

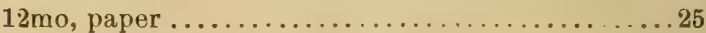

FLEMING. "Operative Veterinary Surgery." Part I, by Dr. Geo. Fleming, M.R.C.V.S. This valuable work, the most practical treatise yet issued on the subject in the English language, is devoted to the common operations of Veterinary Surgery; and the concise descriptions and directions of the text are illustrated with numerous wood engravings. 8 vo, cloth.2 75

Orders are now received for the second rolume, which is expected by December. 
851-8.53 Sixth Avenue (cor. 48th St.), New York. 7

FLEMING. "Human and Animal Variolæ." A Study in Comparative Pathology. Paper ........25

FILEING. "Animal Plagues." Their History, Nature, and Prevention. By George Fleming, F.R.C. V.S., etc. First Series. 8vo, cloth, $\$ 6.00$; Second Series. $8 \mathrm{vo}$, cloth........................ 300

FLEMING. "Roaring in Horses." By Dr. George Fleming, F.R C. $\nabla . S$. A treatise on this peculiar disorder of the Horse, indicating its method of treatment and eurability. 8vo, cloth, with col. plates.1 50

(**) FLEMING-NEUMANN. "Parasites and Parasitic Diseases of the Domesticated Animals." A work which the students of human or veterinary medicine, the sanitarian, agriculturist or breeder or rearer of animals, may refer for full information regarding the external and internal Parasites-vegetable and animal-which attack various species of Domestic Animals. A Treatise by L. G. Neumann, Professor at the National Veterinary School of Toulouse. Translated and edited by George Fleming, C. B., L.L. D.,F.R.C.V.S. 873 pages, 365 illustrations, eloth.7 50

(*) FRIEDBERGER - FROHNER. "Pathology and Therapentics of the Domesticated Animals." By Dr. Friedberger. Translated by Prof. W. L. Zuill, M.D., D.V.S. 2 vol......................1200

GRESSWELL. "The Diseases and Disorders of the 0x." By George Gresswell, B.A. With Notes by James B. Gresswell. Crown, 8vo, eloth, illus....3 50

GRESSWELL. "Diseases and Disorders of the Horse." By Albert, James B., and George Gresswell. Crown, 8vo, illustrated, cloth ...............1 75 
GRESSWELL. Manual of "The Theory and Practice of Equine Medicine." By J. B. Gresswell, F.R.C.V.S., and Albert Gresswell, M.R.C.V.S., second edition, enlarged, 8vo, cloth......................2 75

GRESSWELL. "Veterinary Pharmacology and Therapentics." By James B. Gresswell, F.R.C.V.S. $16 \mathrm{mo}$, eloth.......................... 50

GRESSWELL. "The Bovine Prescriber." For the use of Veterinarians and Veterinary Students. By James B. and Albert Gresswell, M.R.C.V.S Cloth.75

GRESSWELL. "The Equine Hospital Prescriber." drawn up for the use of Veterinary Practitioners and Students. By Drs. James B. and Albert Gresswell, M.R.C.V.S. Cloth........................75

GRESSWELL. "Veterinary Pharmacopæia, Materia Medica and Therapentics." By George and Charles Gresswell, with descriptions and physiological actions of medicines. By Albert Gresswell. Crown,8vo,cl. 275

(**)GOTTHEIL. " "A Manual of General Histology." By Wm. S. Gottheil, M.D., Professor of Pathology in the American Veterinary College, New York; etc., etc.

Histology is the basis of the physician's art, as Anatomy is the foundation of the surgeon's science. Only by knowing the processes of life can we understand the changes of disease and the action of remedies; as the architect must know his building materials, so must the practitioner of medicine know the intimate structure of the body. To present this knowledge in an accessible and simple form has been the author's task. 8vo., cloth, 148 pages, fully illustrated... 100 
851-853 Sixth Avenue (cor. 48th St.), New York. 9

$\left({ }^{*}\right)$ H ASSLOCH. "A Compend of Veterinary Materia Medica and Therapenties." By Dr. A. C. Hassloch, V.S., Lecturer on Materia Medica and Therapeutics, and Professor of Veterinary Dentistry at the NewYork College of Veterinary Surgeons and School of Comparative Medicine, N. Y. 12mo, cloth, pages..

HATES. "Veterinary Notes for Horse-0wners." An every day Horse Book. Jllustrated. By M. H. Hayes. $12 \mathrm{mo}$, cloth........................... 00

HAYES. "Riding:" On the Flat and Across Country. A Guide to Practical Horsemanship. By Captain M. H. Hayes. Second edition, $16 \mathrm{mo}$, cloth.......4 25

HAYES. "Mllustrated Horse Breaking." By Captain M. H. Hares. 12mo, cloth, illustrated.......8 40

HAYES. "The Horsewoman." By Captain M. H. Hayes and Mrs. Hayes. 12mo, cloth, illustrated.4 25

HEATLEY. "The Horse Owner's Safeguard." A handy Medical Guide for every Horse Owner. 12mo, cloth .................................150

HEATLEY. "Practical Veterinary Remedies." $12 \mathrm{mo}$, cloth............................ 00

(**)HEATLEY. "The Stock Owner's Guide." A handy Medical Treatise for every man who owns an ox or cow. By George S. Heatley, M.R.C.V. 12mo,

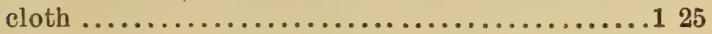

HILL. "The Principles and Practice of Bovine Medicine and Surgery." By J. Woodroffe Hill, F.R.C.V.S. Cloth. (Temporarily out of print). 
HILL. "The Management and Diseases of the Dog." Containing full instructions for Breeding, Rearing and Kenneling Dogs. Their Different Diseases. How to detect and how to cure them. Their Medicines, and the doses in which they can be safely administered. By J. Woodroffe Hill, F.R.C.V.S. 12mo, cloth, extra fully illustrated........................200

HINEBAUCH. "Veterinary Dental Surgery." For the use of Students, Practitioners and Stockmen. $12 \mathrm{mo}$, cloth, illustrated................... 00 Sheep............................ 275

${ }^{(*)}$ HOARE. "A Manual of Veterinary Therapeutics and Piarmacology." By E. Wallis Hoare, F.R.C.V.s. $12 \mathrm{mo}$, cloth, 560 pages.....................2 75

"Deserves a good place in the libraries of all veterinarians. *** Cannot help but be of the greatest assistance to the young veterinarian and the every day busy practitioner."-American Veterinary Revievo.

$\left({ }^{*}\right)$ KOBERT. ${ }^{66}$ Practical Toxicology for Physicians and Students." By Prof. Dr. Rudolph Kobert, Director of the Pharmacological Institute, Dorpat, Kussia. Translated and edited by L. H. Friedburg, Ph.D., of Dept. of Chemistry, College of City of New York, Prof. of Chemistry and Toxicology at the American Veterinary College, New York, and New York Homœathic Medical College and Hospital. Authorized edition. (In Press.)

КОСН. "Etiology of Tuberculosis." By Dr. R.

Koch. Translated by T. Saure. 8vo, cloth ....1 00 
851-853 Sixth Avenue (cor. 48th St.), New York. 11

KEATING. "A New Unabridged Pronouncing Dictionary of Medicine." By John M. Keating, M.D., LL.D., Henry Hamilton and others. A voluminous and exhaustive hand-book of Medical and scientific terminology with Phonetic Pronunciation, Accentuation, Ftymology, etc. With an appendix containing important tables of Bacilli, Microcei Leucomaines, Ptomaines; Drugs and Materials used in Antiseptic Surgery; Poisons and their antidotes; Weights and Measures; Themometer Scales; New Officinal and Unofficinal Drugs, etc., etc. 8 vo, 818 pages.....5 00

\section{LAMBERT. "The Germ Theory of Disease."} Bearing upon the health and welfare of man and the domesticated animals. By James Lambert, F.R.C.V.S. 8 vo. paper............................... 25

$\boldsymbol{L} \boldsymbol{A} \boldsymbol{W}$. "Farmers' Veterinary Adviser." A Guide to the Prevention and Treatment of Disease in Domestic Animals. By Professor James Law.Illustrated. 8vo, cloth .................................. 00

LIAUTARD. "Animal Castration." A concise and practical Treatise on the Castration of the Domestic Animals. The only work on the subject in the English language. Illustrated with forty-four cuts. 12mo, cloth ............................... 00

(") LIAUTARD. " Median Neurotomy." 8vo, cloth .............................. 00

LIAUTARD. " "Vade Mecum of Equine Anatomy." By A. Liautard, M.D.V.S. Dean of the American Veterinary College, $12 \mathrm{mo}$, cloth ..............2 00 
12 Veterinary Catalogue of William R. Jenkins

LIAUTARD. "6ranslation of Zundel on the Horse's Foot." Cloth....................2 00

LIAUTARD. "How to Tell the Age of the Domestic Animal." By Dr. A. Liautard, M.D., V.S. Profusely illustrated. 12mo, cloth...................50

LIAUTARD. "On the Lameness of Horses." By A. Liautard, M.D.,V.S...................250

${ }^{(*)}$ LI AUTARD. "Manual of Operative Veterinary Surgery." By A. Liautard, M.D., V.M., Principal and Professor of Ariatomy, Surgery, Sanitary Meaicine and Jurisprudence in the American Veterinary College; Chevalier du Merite Agricole de France, Honorary Fellow of the Royal College of Veterinary Surgeons (London), etc., etc. 8vo, eloth, 786 pages and nearly 600 illustrations........................6 00

LONG. "Book of the Pig." Its selection, Breeding, Feeding and Management. 8vo, cloth ......... 25

(**)IUPTON. "Horses : Sound and Unsound," with Law relating to Sales and Warranty. By J. Irvine Lupton, F.R.C.V.S. 8vo, cloth, illustrated......1 25

LUPTON. "The Horse." As he Was, as he Is, and as he Ought to Be. By J. I. Lupton, F.R.C.V.S. Illustrated. Crown, 8 vo ....................... 40

MAGNER. "Facts for Horse Owners." By D. Magner. Upwards of 1,000 pages, illustrated with 900 engravings. $8 v 0$, cloth, $\$ 5.00$; sheep, $\$ 6.00$; full morocco.. ......................... 50 
851-853 Sixth Avenue (cor. 48th St.), New York. 13

MA GNER. "Veterinary Diagrams." (1) The Structure of Horses Feet (in colors). The Structure of Horses Feet (Effects of Bad Treatment of the Feet). Mounted and Varnished....................200

(2) The Shoeing of the Horse. The Education of the Horse. Mounted and Varnished..............200

MAYHEW. "The Hlustrated Horse Doctor." An accurate and detailed account of the Various Diseases to which the Equine Race is subject; together with the latest mode of Treatment, and all the Requisite Prescriptions written in plain English. By E. Edward Mayhew, M.R.C.V.S. Illustrated. Entirely new edition, 8 vo, cloth....................... 75

McBRIDE. "Anatomical Outlines of the Horse." $12 \mathrm{mo}$, cloth............................ 50

Mc COMBIE. "Cattle and Cattle Breeders." Cloth.1 00

M'FADYEAN. "Anatomy of the Horse." A Dissection Guide. By J. M. M'Fadyean, M.R.C.V.S. This book is intended for Veterinary students, and offers to them in its 48 full-page colored plates numerous other engravings and excellent text, the most valuable and practical aid in the study of Veterinary Anatomy, especially in the dissecting room. 8vo, cloth .............................. 50

M'FADYEAN. "Comparative Anatomy of the Domesticated Animals." By J. M'Fadyean. Profusely illustrated, and to be issued in two parts. Part I-Osteology, ready. Paper, $\$ 250$; cloth......2 75

(Part II. in preparation.) 
MILLS. "How to Keep a Dog in the City." By Wesley Mills, M.D., V.S. It tells how to choose manage, house, feed, educate the pup, how to keep him clean and teach him eleanliness. Paper.........25

$\left.{ }^{*}\right)$ MOLLER. "Operative Veterinary Surgery." By Professor Dr. H. Moller, Berlin. Translated and edited from the $2 d$ edition, enlarged and improved, by John A. W. Dollar, M.R.C.S.

Prof. Moller's work presents the most recent and complete exposition of the Principles and Practice of Veterinary Surgery, and is the standard text-book on the subject throughout Germany.

Many subjects ignored in previous treatises on Veterinary Surgery here receive full consideration, while the better known are presented under new and suggestive aspects.

As Prof. Moller's work represents not only his own opinions and practice, but those of the best Veterinary Surgeons of various countries, the translation cannot fail to be of signal service to American and British Veterinarians and to Students of Veterinary and Comparative Surgery.

1 vol., 8vo. 722 pages, 142 illustrations ......5 25

MORETON. " On Horse-breaking." $12 \mathrm{mo}, \mathrm{cl} \ldots 50$

MOSSELMA N-LIENA UX. "Veterinary Microbio" logy." By Professors Mosselman and Liénaux, Nationai Veterinary College, Cureghem, Belgium. Translated and edited by R. R. Dinwiddie, Professor of Veterinary Science, College of Agriculture, Arkansas State University. $12 \mathrm{mo}$, cloth, 342 pages......2 50 
851-853 Sixth Avenue (cor. 48th St.), New York. 15

$\left({ }^{*}\right)$ NOCARD. "The Animal Tuberculoses, and their Relation to Human Tuberculosis." By Ed. Nocard, Professor of the Alfort Veterinary College. Translated by H. Scurfield, M.D. Ed., Ph. Camb.

Perhaps the chief interest to doctors of human medicine in Professor Nocard's book lies in the demonstration of the small part played by heredity, and the great part played by contagion in the propagation of bovine tuberculosis. It seems not unreasonable to suppose that the same is the ease for human tuberculosis, and that, if the children of tuberculosis parents were protected from infection by cohabitation or ingestion, the importance of heredity as a cause of the disease, or even of the predisposition to it, would dwindle away into insignificance. 12mo, eloth. 143

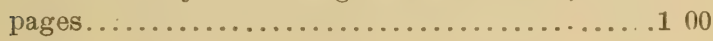

PEGLER. "6The Book of the Goat." 12mo, cloth.1 75

PELLERIN. "Median Nenrotomy in the Treatment of Chronic Tendinitis and Periostosis of the Fetluck." By C. Pellerin, late repetitor of Clinic and Surgery to the Alfort Veterinary School. Translated, with Additional Facts Relating to It, by Prof. A. Liautard, M.D., V.M. 8vo, boards, illustruted.............100

PROCTOR. "The Management and Treatment of the Horse" in the Stable, Field and ou the Road. By William Proctor. 8 vo................. 240

\section{PETERS. "A Tuberculous Herd-Test with Tuber-} culin." By Austin Peters, M. R. C. V.S., Chief Inspector of Cattle for the New York State Board of Health during the winter of 1892-93. Pamphlet...25 
REYNOLD. "Breeding and Management of Draught Horses." 8vo, cloth...................... 40

\section{ROBERTSON. "The Practice of Equine Medicine."} A text-book especially adapted for the use of Veterinary students and Veterinarians. By W. Robertson, Principal and Professor of Hippopathology in the Royal Veterinary College, London. 8vo. cloth, 806 pages, revised edition.................... 25

(**)ROBERGE. "The Foot of the Horse," or Lameness and all Diseases of the Feet traced to an Unbalanced Foot Bone, prevented or cured by balancing the foot. By David Roberge. 8vo, cloth .........5 00

(*)SMITH. "A Manual of Veterinary Physiology." By Veterinary Captain F. Smith, M.R.C.V.S. Author of "A Manual of Veterinary Hygiene."

Throughout this manual the object has been to condense the information as much as possible. The broad facts of the sciences are stated so as to render them of use to the student and practitioner. In this second edition-rewritten-the whole of the Nervous System has been revised, a new chapter dealing with the Development of the Ovum has been added together with many additional facts and illustrations. About one hundred additional pages are given. Second edition, revised and enlarged, with additional illustrations ............................. 75

(**)SMITH. "Manual of Veterinary Hygiene." 2nd edition, revised. Crown, 8vo, cloth ..........3 25 
851-853 Sixth Avenue (cor. 48th St.), New York.

STORNMOUTH. "Manual of Scientific Terms." Especially referring to those in Botany, Natural History, Medical and Veterinary Science. By Rev. James Stornmouth.......................3 00

("*) STRA NGEWAY. "Veterinary Anatomy." New edition, revised and edited by I. Vaughn, F.L.S., M.R.C.V.S., with several hundred illustrations. 8vo, cloth ............................. 00

(*)SUSSDORF. Colored Plates specially for Lectures. Size 40x27. By Professor Sussdorf, M.D. Translated by Prof. W. Owen Williams, of the New Veterinary College, Edinburgh. Plate 1.-Diagram of the Horse (left or near side view): Plate 2.-Diagram of the Mare (right side view). Showing the position of the viscera in the large cavities of the body.

Price, unmounted...................1 75 each

"s mounted on linen, with roller...1 75 extra "

"Anatomy of the Cow" (in preparation).

VETERINARY DIAGRAMS in Tabular Form. Size, $28 \frac{1}{2}$ in. $x 22$ inches. Price per set of five...4 75

No. 1. "The External Form and Elementary Anatomy of the Horse." Eight coloured illustrations1. External regions; 2. Skeleton; 3. Muscles (Superior Layer); 4. Muscles (Deep Layer); 5. Respiratory Apparatus; 6. Digestive Apparatus; 7. Circulatory Apparatus ; 8. Nerve Apparatus ; with letter-press descrip-

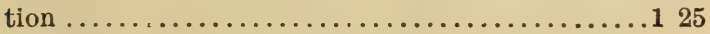

No. 2. "The Age of Domestic Animals." Forty-two figures illustrating the structure of the teeth, indicating the Age of the Horse; Ox, Sheep, and Dog, with full description .........................75 
No. 3. "The Unsoundness and Defects of the Horse." Fifty figures illustrating-1. The Defects of Conformation; 2. Defects of Position; 3. Infirmities or Signs of Disease; 4. Unsoundnesses; 5. Defects of the Foot; with full description..................... 75

No. 4. "The Shoeing of the Horse, Mu'e and 0x." Fifty figures descriptive uf the Anatomy and Physiology of the Foot and of Horse-shoeing.

No. 5. "The Elementary Anatomy, Points, and Butcher's Joints of the Ox." Ten coloured illustrations -1. Skeleton; 2. Nervous System: 3. Digestive System (Right Side); 4. Respiratory System ; 5. Points of a Fat Ox; 6. Muscular System; 7. Vascular System; 8. Digestive System (Left Side); 9. Butcher's Sections of a Calf ; 10. Butcher's Sections of an Ox; with full description ............................ 25

WALLEY. "Hints on the Breeding and Rearing of Farm Animals." 12mo, eloth...............8u

WALLEY. " Fonr Bovine Scourges." (Pleuro-Pneumonia, Foot and Mouth Disease, Cattle Plague and Tubercle.) With an Appendix on the Inspection of Live Animals and Meat. Illustrated, 4to, cloth. 640

WALLEY. "The Horse, Cow and Dog." By Dr. Thomas Walley. A poetical account of the "Troublous Life of the Horse"; "The Life of a Dairy Cow," and "The Life of a Dog" ; with an article on Animal Characteristics, 12mo, cloth, ...............80 
(*) W AL LEY. "A Practical Guide to Meat Inspection." By Thomas Walley, M.R.C.V.S., formerly principal of the Edinburgh Royial (Dick) Veterinary College: Professor of Veterinary Medicine and Surgery, etc. Third Edition, thoroughly revised, with forty-five coloured illustrations, $12 \mathrm{mo}$, cloth.............300

An experience of over 30 years in his profession and a long official connection (some sixteen years) with Edinburgh Abattoirs have enabled the author to gather a large store of information on the subject, which he has embodied in his book. Dr. Walley's opinions are regarded as the highest authority on Meat Inspection.

$\left(^{* *}\right)$ WILLIAMS. "Principles and Practice of Veterinary Medicine." New author's edition, entirely revised and illustrated with numerous plain and colored plates. By W. Williams, M.R.C.V.S.8vo., cl..60 00

(**)WILLIAMS. "Principles and Practice of Veter" inary Surgery." New author's edition, entirely revised and illustrated with numerous plain and colored plates. By W. Williams, M.R.C.V.S. 8vo, cloth..............................6 00

ZUNDEL. “The Horse's Foot and Its Diseases." By A. Zundel, Principal Veterinarian of Alsace Lorraine. Translated by Dr. A. Liautard, V.S. 12mo, cloth illustrated........................... 00

ZUILL. "Typhoid Ferer; or Contagious Influenza in the Horse." By Prof. W. L. Zuill, M.D.,D.V.S.

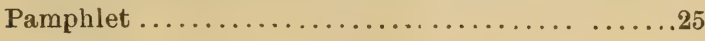








